\title{
Neutrino. History of a unique particle
}

\author{
S. M. Bilenky \\ Joint Institute for Nuclear Research, Dubna, R-141980, Russia \\ TRIUMF 4004 Wesbrook Mall, Vancouver BC, V6T $2 A 3$ Canada
}

\begin{abstract}
Neutrinos are the only fundamental fermions which have no electric charges. Because of that neutrinos have no direct electromagnetic interaction and at relatively small energies they can take part only in weak processes with virtual $W^{ \pm}$and $Z^{0}$ bosons (like $\beta$-decay of nuclei, inverse $\beta$ process $\bar{\nu}_{e}+p \rightarrow e^{+} n$, etc.). Neutrino masses are many orders of magnitude smaller than masses of charged leptons and quarks. These two circumstances make neutrinos unique, special particles. The history of the neutrino is very interesting, exciting and instructive. We try here to follow the main stages of the neutrino history starting from the Pauli proposal and finishing with the discovery and study of neutrino oscillations.
\end{abstract}

\section{The idea of neutrino. Pauli}

\section{Introduction}

The history of the neutrino started with the famous Pauli letter. The experimental data "forced" Pauli to assume the existence of a new particle which later was called neutrino. The hypothesis of the neutrino allowed Fermi to build the first theory of the $\beta$-decay which he considered as a process of a quantum transition of a neutron into a proton with the creation of an electron-(anti)neutrino pair. During many years this was the only experimentally studied process in which the neutrino takes part. The main efforts were devoted at that time to the search for a Hamiltonian of the interaction which governs the decay.

The hypothesis of neutrino was proposed by W. Pauli in December 1930 in the famous letter addressed to participants of a nuclear conference in Tübingen. At that time protons and electrons were considered as elementary particles and nuclei were considered as bound states of protons and electrons. In the framework of this last assumption there were two fundamental problems: 
1. The problem of continuous $\beta$ spectra.

2. The problem of spin of some nuclei.

From the point of view of the proton-electron model the $\beta$-decay of a nucleus $(A, Z)$ is a process of emission of an electron in the nuclear transition $(A, Z) \rightarrow(A, Z+1)+e^{-}$. From the conservation of the energy and momentum it followed in this case that the electron produced in the $\beta$ decay had a fixed kinetic energy, approximately equal to the released energy $Q=\left(M_{A, Z}-M_{A, Z+1}\right)-m_{e}$. In experiments, however, continuous $\beta$-spectra with an end-point energy equal to $Q$ were observed.

There was a belief that continuous $\beta$ - spectra could be explained by the loss of energy of electrons in the target. However, in 1927 Ellis and Wooster performed a calorimetric $\beta$-decay experiment [1]. They found that the energy per $\beta$-decay of a nucleus was equal to the energy averaged over the $\beta$-spectrum. Thus, it was proved that the energy detected in the $\beta$-decay was smaller than the total released energy.

After the Ellis and Wooster experiment the situation with the continuous $\beta$-spectrum became dramatic. Pauli was the first who understood that under the condition of energy-momentum conservation the only possibility to explain the continuous $\beta$-spectra was to assume that there existed a new, neutral particle which was emitted in the $\beta$-decay together with the electron and is not detected in an experiment. Pauli called the new particle the neutron. Let us notice that there was at that time also an idea that energy in the $\beta$-decay is not conserved (A. Bohr).

If the $\beta$-decay is a three-body process

$$
(A, Z) \rightarrow(A, Z+1)+e^{-}+" n ",
$$

the released energy is shared between the electron and the "neutron", and a continuous electron spectrum will be observed. As in $\beta$-experiments only electrons were detected, Pauli assumed that the absorption length of the "neutron" was "the same or probably 10 times larger than the absorption length of the $\gamma$-quantum" 1 Pauli further assumed that "neutrons" had spin $1 / 2$ and together with electrons and protons were constituents of nuclei. This

\footnotetext{
${ }^{1}$ Pauli suggested in the letter that the "neutron" had a magnetic moment $\mu$. He wrote: "The experiments seem to require that the ionizing effect of such a neutron can not be bigger than the one of a gamma-ray, and then $\mu$ is probably not allowed to be larger than $10^{-13} \mathrm{e} \cdot \mathrm{cm} "$.
} 
allowed him to solve another problem which existed at that time, the problem of the spin of some nuclei.

Let us consider the ${ }^{14} \mathrm{~N}_{7}$ nucleus. From the point of view of the electronproton model this nucleus is a bound state of 14 protons and 7 electrons, i.e. the spin of ${ }^{14} \mathrm{~N}_{7}$ nucleus has to be half-integer. However, from the investigation of spectra of molecular nitrogen it followed that ${ }^{14} \mathrm{~N}_{7}$ nuclei satisfied the Bose-Einstein statistics. Thus, according to the general theorem on the connection between spin and statistics the spin of the ${ }^{14} \mathrm{~N}_{7}$ nucleus must be integer. If in addition to electrons and protons the spin $1 / 2$ "neutrons" are also constituents of nuclei, the spin of ${ }^{14} \mathrm{~N}_{7}$ can be integer.

Pauli also assumed that the "neutron", a constituent of nuclei, must have a mass different from zero. He wrote in the letter that "the mass of the neutrons should be of the same order of magnitude as the electron mass and in any event not larger than 0.01 of the proton mass" 2

In 1932 the neutron, a heavy particle with the mass practically equal to the mass of the proton, was discovered by J. Chadwick [2]. Soon after this discovery Heisenberg [3], Majorana [24] and Ivanenko [5] put forward a hypothesis that nuclei are bound states of protons and neutrons. This hypothesis could successfully describe all nuclear data. The problem of the spin of ${ }^{14} \mathrm{~N}_{7}$ and other nuclei disappeared $\left({ }^{14} \mathrm{~N}_{7}\right.$ nucleus is the bound state of 7 protons and 7 neutrons and has an integer spin in accordance with the theorem on the connection between spin and statistics.)

\section{Neutrino and the first theory of the $\beta$-decay}

The next fundamental contribution to the development of the idea of the neutrino was made by E. Fermi in 1934 [6]. Fermi built the first theory of the $\beta$-decay of nuclei. The theory was based on the Pauli assumption that in the $\beta$-decay together with the electron a neutral, spin $1 / 2$, light particle was emitted.

After the discovery of the heavy neutron Fermi proposed to call the light

\footnotetext{
${ }^{2}$ Pauli was the first who suggested the existence of a new particle which was not directly observed in an experiment (but was needed for the explanation of the experimental data). Nowadays it is common practice but in Pauli's time it was a very courageous proposal. It is interesting that in the framework of the wrong electron-proton-"neutron" model of nuclei Pauli correctly predicted that the new particle emitted in the $\beta$-decay was a particle with spin $1 / 2$ and nonzero mass.
} 
Pauli particle the neutrino (from Italian neutral, light). Fermi built the theory of the $\beta$-decay assuming that nuclei are bound states of protons and neutrons. There was a problem to understand how an electron-neutrino pair was produced. By analogy with the emission of a photon by an electron Fermi assumed that the electron-neutrino pair is produced in the quantum transition of a neutron into a proton 3

$$
n \rightarrow p+e^{-}+\bar{\nu}
$$

The simplest electromagnetic Hamiltonian which induces the quantum transition

$$
p \rightarrow p+\gamma
$$

has the form of the scalar product of the electromagnetic (vector) current $\bar{p}(x) \gamma_{\alpha} p(x)$ and vector electromagnetic field $A^{\alpha}(x)$

$$
\mathcal{H}^{\mathrm{EM}}(x)=e \bar{p}(x) \gamma_{\alpha} p(x) A^{\alpha}(x)
$$

By analogy Fermi assumed that the Hamiltonian of the decay (1) was the scalar product of the vector $\bar{p}(x) \gamma_{\alpha} n(x)$ and the vector $\bar{e}(x) \gamma_{\alpha} \nu(x)$ which could be built from electron and neutrino fields 4

$$
\mathcal{H}^{\beta}(x)=G_{F} \bar{p}(x) \gamma_{\alpha} n(x) \bar{e}(x) \gamma_{\alpha} \nu(x)+\text { h.c. }
$$

where $G_{F}$ is a constant (which is called Fermi constant).

Let us stress the fundamental difference between the Hamiltonian of the electromagnetic interaction (3) and the Hamiltonian of the $\beta$-decay (4). The electromagnetic Hamiltonian $\mathcal{H}^{\mathrm{EM}}$ is the Hamiltonian of the interaction of two fermion fields and a boson field and $\mathcal{H}^{\beta}$ is the Hamiltonian of the interaction of four fermion fields. As a result of that the constants e and $G_{F}$ have different dimensions. In the system of units $\hbar=c=1$, we use, the charge $e$ is a dimensionless quantity and the Fermi constant $G_{F}$ has dimension $M^{-2}$ ( $M$ is a mass). Later we will discuss the origin of the dimension of the constant $G_{F}$. We will see that the dimension of the constant $G_{F}$ means that the

\footnotetext{
${ }^{3}$ We know today that in the $\beta$-decay together with the electron an antineutrino $\bar{\nu}$ is produced. Later we will explain the difference between neutrino and antineutrino.

${ }^{4}$ The current $\bar{p} \gamma_{\alpha} n$ induces the transition $n \rightarrow p$. It changes the electric charge by one $(\Delta Q=1)$ and is called the hadronic charged current (CC). The current $\bar{e} \gamma_{\alpha} \nu$ provides the emission of the pair $\left(e^{-}-\bar{\nu}\right)$. It is called the leptonic CC.
} 
Hamiltonian (4) is not a fundamental Hamiltonian of interaction but is an effective Hamiltonian.

Applying the methods of the Quantum Field Theory and using the Hamiltonian (44), Fermi calculated the spectrum of electrons emitted in the $\beta$-decay and suggested a method of the measurement of the neutrino mass. For that he proposed to investigate the shape of the electron spectrum in the region near the maximal electron energy (which corresponds to the emission of non relativistic neutrinos) 5

It occurred that the investigation of the $\beta$-decay of tritium

$$
{ }^{3} \mathrm{H} \rightarrow{ }^{3} \mathrm{He}+e^{-}+\bar{\nu}
$$

is one the most sensitive ways of the measurement of the neutrino mass by the Fermi-Perrin method 6

The electron spectrum for the allowed transitions is determined by the phase-space factor

$$
p_{e} E_{e} p E,
$$

where $E_{e}$ and $E\left(p_{e}\right.$ and $\left.p\right)$ are the energies (momenta) of the electron and the neutrino.

If we neglect the recoil of the final nucleus, from the conservation of the energy we have

$$
Q=T_{e}+E,
$$

where $T_{e}=E_{e}-m_{e}$ is the kinetic energy of the electron.

From (6) we obtain the following expression for the spectrum of the electrons in the decay (5)

$$
\frac{d \Gamma}{d E}=C p_{e}\left(T_{e}+m_{e}\right)\left(Q-T_{e}\right) \sqrt{\left(Q-T_{e}\right)^{2}-m_{\nu}^{2}} F\left(T_{e}, Z\right),
$$

where $m_{\nu}$ is the neutrino mass, $F\left(T_{e}, Z\right)$ is the Fermi function, which describes the Coulomb interaction of the final electron and nucleus, and $C$ is a constant (which includes the modulus-squared of the nuclear matrix element).

The neutrino mass enters into the expression for the $\beta$-spectrum through the neutrino momentum $p=\sqrt{\left(Q-T_{e}\right)^{2}-m_{\nu}^{2}}$. From this expression it is

\footnotetext{
${ }^{5}$ The same method of the measurement of the neutrino mass was proposed by Perrin 7 .

${ }^{6}$ This is connected with the fact that tritium has a convenient half-life $T_{1 / 2}=12.3$ years, the energy release in the process (5) is small $(Q=18.57 \mathrm{keV})$, the nuclear matrix element of the process is a constant $\left({ }^{3} \mathrm{H} \rightarrow{ }^{3} \mathrm{He}\right.$ is an allowed transition), etc.
} 
obvious that the part of the spectrum in which $Q-T_{e} \lesssim m_{\nu}$ is sensitive to the neutrino mass 7

The largest contributions to the $\beta$-decay come from transitions in which electron and (anti)neutrino are produced in states with orbital momenta equal to zero ( $S$-states) 8 Such transitions are called allowed. For allowed transitions it follows from the Fermi Hamiltonian (4) that spins and parities of the initial and final nuclei must be equal (Fermi selection rules):

$$
\Delta J=0, \quad \pi_{i}=\pi_{f} .
$$

Here $\Delta J=J_{f}-J_{i}\left(J_{i}\left(J_{f}\right)\right.$ is the spin of the initial (final) nucleus) and $\pi_{i}$ $\left(\pi_{f}\right)$ is the parity of the initial (final) nucleus.

From the conservation of the total momentum it follows that in the case of an allowed transition which satisfies the Fermi selection rule electron and (anti)neutrino are produced in a state with the total spin $S$ equal to zero (singlet state). If electron and (anti)neutrino are produced in the triplet state $(S=1)$ in this case for the allowed transition the total angular momentum of the final state is equal to $J_{f}=J_{i} \pm 1$ or $J_{f}=J_{i}$ (for $J_{i}=0$ the total final angular momentum is equal to 1 ). We have in this case

$$
\Delta J= \pm 1,0 \quad \pi_{i}=\pi_{f} \quad(0 \rightarrow 0 \text { is forbidden }) .
$$

The selection rules (10) are called the Gamov-Teller selection rules. They were introduced by Gamov and Teller in 1936 [8].

In the $\beta$-decay experiments, decays of nuclei which satisfy the Fermi and Gamov-Teller selection rules were observed. Thus, the total Hamiltonian of the $\beta$-decay must include not only the Fermi Hamiltonian (4) but also an additional term (or terms).

The Fermi Hamiltonian is the product of vector $\times$ vector. The most general Hamiltonian of the Fermi type, in which only fields but not their derivatives enter, has the form of the sum of the products of scalar $\times$ scalar, vector $\times$ vector,

\footnotetext{
${ }^{7}$ In practice, for a neutrino mass $m_{\nu} \lesssim 1 \mathrm{eV}$ a much larger part of the spectrum is used for the analysis of experimental data (in order to increase the luminosity of the experiment).

${ }^{8}$ The neutrino and the electron are produced in the $\beta$-decay in states with definite momenta. Their wave function has the form $e^{i \overrightarrow{p_{e}} \vec{x}+i \vec{p} \vec{x}}$. We have $\vec{p} \vec{x} \leq p R$. where $R \simeq 1.2 \cdot 10^{-13} A^{1 / 3} \mathrm{~cm}$ is the radius of a nucleus. Taking into account that the energies of the neutrino and the electron produced in the $\beta$-decay are not larger than a few $\mathrm{MeV}$, we have $|\vec{p} \vec{x}| \ll 1$ and $\left|\vec{p}_{e} \vec{x}\right| \ll 1$. Thus, in the first approximation $e^{i \vec{p}_{e} \vec{x}+i \vec{p} \vec{x}} \simeq 1$. This approximation corresponds to the emission of the electron and the neutrino into $S$-states (allowed transition).
} 
tensor $\times$ tensor, axial $\times$ axial and pseudoscalar $\times$ pseudoscalar:

$$
\mathcal{H}_{I}^{\beta}(x)=\sum_{i=S, V, T, A, P} G_{i} \bar{p}(x) O^{i} n(x) \bar{e}(x) O_{i} \nu(x)+\text { h.c. }
$$

Here

$$
O^{i} \rightarrow 1(S), \gamma^{\alpha}(V), \sigma^{\alpha \beta}(T), \gamma^{\alpha} \gamma_{5}(A), \gamma_{5}(P)
$$

and $G_{i}$ are coupling constants, which have dimensions $[M]^{-2}$. 9 The Hamiltonian (11) describes all $\beta$-decay data. Transitions, which satisfy the Fermi selection rules, are due to $V$ and $S$ terms and transitions which satisfy the Gamov-Teller selection rules, are due to $A$ and $T$ terms.

In the Fermi Hamiltonian (41) only one fundamental constant $G_{F}$ enters. The Hamiltonian (11) is characterized by five (!) interaction constants. Analogy and economy which were the basis of the Fermi theory were lost.

There was a general belief that there are "dominant" terms in the interaction (11). Such terms were searched for many years via analysis of the data of different $\beta$-decay experiments. This search did not lead, however, to a definite result: some experiments were in favor of $V$ and $A$ terms, other were in favor of $S$ and $T$ terms. Up to 1957 when the violation of parity in the $\beta$-decay (and other weak processes) was discovered, the situation with the Hamiltonian of the $\beta$-decay remained uncertain.

\section{The first estimate of the neutrino-nucleus cross section}

\section{Introduction}

Very early physicists started to think about a possibility to detect the neutrino and thus to prove directly its existence. However, in the thirties and the forties there were no technical possibilities to do this. We will discuss here the first paper in which the neutrino-nucleous cross section was estimated.

\footnotetext{
${ }^{9}$ Dirac matrices $\gamma^{\alpha}(\alpha=0,1,2,3)$ satisfy the relations $\gamma^{\alpha} \gamma^{\beta}+\gamma^{\beta} \gamma^{\alpha}=2 g^{\alpha \beta}$, where $g^{00}=$ $1, g^{i i}=-1$ and non diagonal elements of $g^{\alpha \beta}$ are equal to zero. The matrix $\gamma_{5}$ is determined as follows $\gamma_{5}=-i \gamma_{0} \gamma_{1} \gamma_{2} \gamma_{3}$. It satisfies the relations $\gamma^{\alpha} \gamma_{5}+\gamma_{5} \gamma^{\alpha}=0, \gamma_{5} \gamma_{5}=1$. Sixteen matrices $1, \gamma^{\alpha}, \sigma_{\alpha \beta}=\frac{1}{2}\left(\gamma^{\alpha} \gamma^{\beta}-\gamma^{\beta} \gamma^{\alpha}\right), \gamma^{\alpha} \gamma_{5}, \gamma_{5}$ form a complete system of $4 \times 4$ matrices.
} 
In the Fermi Hamiltonian (4) $e(x), \nu(x), n(x)$ and $p(x)$ are quantum fields. This means that the Hamiltonian (44) allows one to calculate not only the probability of the $\beta^{-}$-decay

$$
(A, Z) \rightarrow(A, Z+1)+e^{-}+\bar{\nu}
$$

but also the probabilities of the $\beta^{+}$-decay and electron capture

$$
(A, Z) \rightarrow(A, Z-1)+e^{+}+\nu, \quad e^{-}+(A, Z) \rightarrow \nu+(A, Z-1),
$$

the cross sections of the neutrino reactions

$$
\begin{gathered}
\bar{\nu}+(A, Z) \rightarrow e^{+}+(A, Z-1), \\
\nu+(A, Z) \rightarrow e^{-}+(A, Z+1)
\end{gathered}
$$

and other processes.

The first estimation of the cross section of the process (15) was obtained by Bethe and Peierls [9] soon after the Fermi theory was proposed.

We will present here Bethe's and Peierls's arguments. At relatively small $\mathrm{MeV}$ energies the nuclear matrix elements of the processes (13) and (15) are practically the same. Since the $\beta$-decay width $\Gamma=\frac{1}{T_{1 / 2}}\left(T_{1 / 2}\right.$ is the half-life of the decay) and the cross section $\sigma$ of the process (14) are proportional to the modulus-squared of the nuclear matrix elements, we have

$$
\sigma=\frac{A}{T_{1 / 2}}
$$

where $A$ has dimension (length) $)^{2} \times$ time. The authors suggested that "the longest length and time which can possibly be involved are $\frac{\hbar}{m_{e} c}$ and $\frac{\hbar}{m_{e} c^{2}}$ " and found the following bound

$$
\sigma<\frac{\hbar^{3}}{m_{e}^{3} c^{4} T_{1 / 2}}
$$

From this inequality for $T_{1 / 2} \simeq 3$ min Bethe and Peierls found

$$
\sigma<10^{-44} \mathrm{~cm}^{2} .
$$

This bound corresponds to a neutrino absorption length in solid matter larger than $10^{14} \mathrm{~km} .10$ On the basis of this estimate Bethe and Peierls in their

\footnotetext{
${ }^{10}$ It is interesting to compare this number with Pauli's original expectation: "... I trustfully turn first to you, dear radioactive people, with the question of how likely it is to find experimental evidence for such a neutron if it would have the same or perhaps a 10 times larger ability to get through [material] than a gamma-ray" (Pauli letter).
} 
paper( with the title The "Neutrino") concluded "...there is no practically possible way of observing the neutrino".

For comparison we will present the current calculations of the neutrino cross section. Let us consider the process

$$
\bar{\nu}+p \rightarrow e^{+}+n .
$$

Using the modern Hamiltonian of the weak interaction for the cross section of the process (20) we have

$$
\sigma=4 \frac{G_{F}^{2}}{\pi} p_{e} E_{e} \simeq 9.5 \cdot 10^{-44} \frac{p_{e} E_{e}}{\mathrm{MeV}^{2}} \mathrm{~cm}^{2},
$$

where $E_{e}$ and $p_{e}$ are the positron energy and the momentum. Neglecting the recoil of the final neutron we have for the neutrino energy $E$

$$
E=E_{e}+\Delta,
$$

where $\Delta=m_{n}-m_{p} \simeq 1.3 \mathrm{MeV}$ is the neutron-proton mass difference. For (anti)neutrinos with the energy $E \simeq 3 \mathrm{MeV}$ we find the value $\sigma \simeq 2.6$. $10^{-43} \mathrm{~cm}^{2}$ from (21). Correspondingly, the absorption length of (anti)neutrinos in water is given by

$$
L_{a}=\frac{1}{n \sigma} \simeq 6 \cdot 10^{14} \mathrm{~km},
$$

where $n$ is the number density of protons (in the case of water $n \simeq 6.7$. $\left.10^{22} \frac{1}{\mathrm{~cm}^{3}}\right)$. Thus, present-day calculations confirm the Bethe and Peierls estimate.

After the Bethe and Peierls paper there was a general opinion that the neutrino is an undetectable particle. The first physicist who challenged this opinion was B. Pontecorvo [10]. In 1946 he proposed a radiochemical method of neutrino detection and in particular the $\mathrm{Cl}-\mathrm{Ar}$ method which is based on the reaction

$$
\nu+{ }^{37} \mathrm{Cl} \rightarrow e^{-}+{ }^{37} \mathrm{Ar} .
$$

Many years later the $\mathrm{Cl}-\mathrm{Ar}$ method of neutrino detection allowed R. Davis to observe solar neutrinos in the first solar neutrino experiment [11]. We will discuss solar neutrinos and the Pontecorvo radiochemical method later. 


\section{First ideas of $\mu-e$ universal weak interac- tion}

\section{Introduction}

With the idea of $\mu-e$ universality there appeared a notion of universal weak interaction. The idea of universality was proposed, however, at the time when the form of the weak interaction was not known. It was, nevertheless, an extremely important general idea. We will see later how it was implemented in the $V-A$ theory of weak interaction.

In 1947 B. Pontecorvo[12] came to an idea of existence of a universal weak interaction which governs not only processes in which the electron-neutrino pair takes part (like the nuclear $\beta$-decay) but also processes in which the muon-neutrino pair participates. The process of such a type is $\mu$-capture

$$
\mu^{-}+(A, Z) \rightarrow \nu+(A, Z-1)
$$

B. Pontecorvo compared the probability of this process and the probability of the $K$-capture

$$
e^{-}+(A, Z) \rightarrow \nu+(A, Z-1)
$$

and came to the qualitative conclusion that the constant of the interaction of the muon-neutrino pair with nucleons is of the same order as the Fermi constant.

The idea of $\mu-e$ universality of the weak interaction was also proposed by G. Puppi [13]. Puppi presented it in the form of a triangle ("Puppi triangle"). He assumed that a universal weak interaction includes not only Hamiltonians of the $\beta$-decay and $\mu$-capture but also the Hamiltonian of the $\mu$-decay

$$
\mu^{+} \rightarrow e^{+}+\nu+\bar{\nu}
$$

Puppi suggested that different parts of the weak interaction form a triangle with vertices

$$
(\bar{p} n)-(\bar{\nu} e)-(\bar{\nu} \mu)
$$

and the Hamiltonian of the weak interaction is given by a sum of products of different vertices. The idea of a universal weak interaction was proposed also by O.Klein [33] and Yang and Tiomno [15]. 


\section{Parity violation in the $\beta$-decay and other weak processes}

\section{Introduction}

Conservation of parity (invariance under space inversion, i.e. under transition from a right-handed to a left-handed system) was firmly established for strong (hadronic) and electromagnetic processes. For many years physicists thought that the invariance under space inversion is a general law of nature valid for all interactions. The discovery of violation of parity in the $\beta$-decay and other weak processes was a great surprise. In the beginning it looked that this discovery made the theory of the $\beta$-decay and other weak processes more complicated. In reality, as we will see later, this discovery allowed building a simple, correct theory of the neutrino and weak interaction.

The violation of parity in the weak interaction was one of the most important discoveries in the physics of the XX century. In 1957 Lee and Yang were awarded the Nobel Prize "for their penetrating investigation of the so-called parity laws which has led to important discoveries regarding the elementary particles".

Our understanding of the neutrino and the weak interaction has drastically changed after it was discovered in 1957 that in the $\beta$-decay, the decay $\mu^{+} \rightarrow e^{+}+\nu+\bar{\nu}$ and other weak processes parity is not conserved.

The investigation of the decays of strange particles at the beginning of the fifties created the so called $\theta-\tau$ puzzle 11 As one of the possible solutions of the $\theta-\tau$ problem Lee and Yang [16] put forward the hypothesis of the non-conservation of parity (1956). They analyzed existing experimen-

\footnotetext{
${ }^{11}$ A strange particle which decays into $\pi^{+}$and $\pi^{0}$ was called $\theta^{+}\left(\theta^{+} \rightarrow \pi^{+}+\pi^{0}\right)$ and a strange particle which decays into $\pi^{+}$and $\pi^{-}$and $\pi^{+}$was called $\tau^{+}\left(\tau^{+} \rightarrow \pi^{+}+\pi^{-}+\pi^{+}\right)$. From experimental data it followed that the masses and lifetimes of $\theta^{+}$and $\tau^{+}$are the same. The study of the Dalitz plot of the decay of $\tau^{+}$showed that the total momentum of the state of $\left(\pi^{+}, \pi^{-}, \pi^{+}\right)$was equal to zero and the parity (eigenvalue of the operator of the parity) was equal to -1 . If $\tau^{+}$and $\theta^{+}$are the same particle in this case its spin must be equal to zero. However, the parity of the two pions produced in the $\theta^{+}$-decay is equal to +1 (the parity of two pions is equal to $I_{\pi}^{2}(-1)^{l}=(-1)^{2}(-1)^{0}=1$, where $I_{\pi}=-1$ is the internal parity of the pion and $l$ is the orbital momentum of two pions). So if $\tau^{+}$and $\theta^{+}$are the same particle (which is natural to assume because of the equality of masses and lifetimes) we are confronted with the following problem: the same particle decays into states with different parities.
} 
tal data and came to the conclusion that there was an evidence that parity is conserved in the strong and electromagnetic interactions, but there were no data which proved that parity was conserved in the $\beta$-decay and other weak decays. ("...as for weak interactions parity conservation is so far only extrapolated hypothesis unsupported by experimental evidence" [16]). Lee and Yang proposed different experiments which would allow to test the hypothesis of the parity conservation in weak decays. The results of the first experiments in which large violation of parity in weak processes was observed were published by $\mathrm{Wu}$ et al. [17] and Lederman et al. [18] at the beginning of 1957.

We will discuss first the experiment by $\mathrm{Wu}$ et al. in which the $\beta$-decay of polarized ${ }^{60} \mathrm{Co}$ was investigated ${ }^{12}$ Let us consider the emission of the electron with momentum $\vec{p}$ in the $\beta$-decay of a nucleus with polarization $\vec{P}$. From the invariance under rotations (conservation of the total momentum) it follows that the decay probability can depend only on the scalar products $\vec{p} \cdot \vec{p}$ and $\vec{P} \cdot \vec{p}$. Taking into account that the decay probability depends linearly on the polarization of a nucleus we obtain the following general expression for the probability of the emission of the electron with momentum $\vec{p}$ by a nucleus with polarization $\vec{P}$

$$
w_{\vec{P}}(\vec{p})=w_{0}(1+\alpha \vec{P} \cdot \vec{k})=w_{0}(1+\alpha P \cos \theta) .
$$

Here $\vec{k}=\frac{\vec{p}}{p}$ is a unit vector in the direction of the electron momentum, $\theta$ is the angle between the vectors $\vec{P}$ and $\vec{p}$, and $w_{0}$ and $\alpha$ are functions of $p^{2}$.

Under the inversion of a coordinate system (change of directions of all axes of the coordinate system) momentum $\vec{p}$ and polarization $\vec{P}$ are transformed differently. Namely, momentum is transformed as a vector

$$
p_{i}^{\prime}=-p_{i}
$$

while polarization is transformed as a pseudovector 13

$$
P_{i}^{\prime}=+P_{i}
$$

Here $p_{i}\left(P_{i}\right)$ are components of a vector of momentum (pseudovector of polarization) in some right-handed system and $p_{i}^{\prime}\left(P_{i}^{\prime}\right)$ are components of the same momentum (same polarization) in the inverted (left-handed) system.

\footnotetext{
${ }^{12}$ Polarization of a nucleus is the average value of its spin.

${ }^{13}$ Notice that momentum, coordinates, electric field etc. are vectors while angular momentum, polarization, magnetic field etc. are pseudovectors.
} 
Relations (30) and (31) mean that under the inversion the vector of momentum does not change its position in space while polarization changes its direction to the opposite one.

From (30) and (31) it follows that under the inversion the scalar product $\vec{P} \cdot \vec{p}$ is transformed as a pseudoscalar (change sign)

$$
\overrightarrow{P^{\prime}} \cdot \overrightarrow{p^{\prime}}=-\vec{P} \cdot \vec{p}
$$

while $\vec{p} \cdot \vec{p}$ is transformed as a scalar

$$
\overrightarrow{p^{\prime}} \cdot \overrightarrow{p^{\prime}}=+\vec{p} \cdot \vec{p} .
$$

If the invariance under the inversion holds (parity is conserved), in this case the decay probability in a right-handed system and in an inverted left-handed system is the same

$$
w_{\overrightarrow{P^{\prime}}}\left(\overrightarrow{p^{\prime}}\right)=w_{\vec{P}}(\vec{p}) .
$$

From (29), (30), (31) and (34) we conclude that in the case of conservation of parity $\alpha=0$ and the probability of the emission of the electron by the polarized nucleus does not depend on the angle $\theta 14$

In the $\mathrm{Wu}$ et al. experiment [17] it was found that the parameter $\alpha$ was negative and $|\alpha| \geq 0.7$ (i.e. electrons are emitted mainly in the direction opposite to the polarization of the nucleus) 15 Thus, it was discovered that there was no invariance of the $\beta$-decay interaction under inversion (parity in the $\beta$-decay is not conserved).

The paper of Wu et al. [17] was submitted to Physical Review on January 15, 1957. The same day another experimental paper [18] on the observation of the violation of parity in weak decays was submitted to the same journal.

\footnotetext{
${ }^{14}$ Let us consider the emission of electrons by a polarized nucleus at the angle $\theta$ in a right-handed system. Conservation of parity means that the probability of the emission of electrons at the same angle $\theta$ in the inverted left-handed system must be the same. However, because polarization is a pseudovector, the probability of the emission of electrons at the angle $\theta$ in the left-handed system is equal to the probability of the emission of electrons in the right-handed system at the angle $\pi-\theta$. Thus, if parity is conserved the probabilities of the emission of the electron at the angles $\theta$ and $\pi-\theta$ must be the same. This means that the pseudoscalar $\vec{P} \cdot \vec{k}=P k \cos \theta$ can not enter into the probability.

${ }^{15}$ The sign of the asymmetry parameter $\alpha$ depends on the handedness of the system. Conservation of parity means that such parameters can not enter into measurable quantities. After $\mathrm{Wu}$ et al. and other experiments we know that this is not the case.
} 
In the Lederman et al. experiment [18] strong violation of parity in the chain of the decays

$$
\pi^{+} \rightarrow \mu^{+}+\nu
$$

and

$$
\mu^{+} \rightarrow e^{+}+\nu+\bar{\nu}
$$

was observed.

If parity is violated, a muon produced in the decay (35) will be polarized in the direction of the muon momentum 16 Like in the case of the $\beta$-decay, the dependence of the probability of the decay of polarized muons on the angle $\theta$ between muon polarization and electron momentum has the general form $(1+a \cos \theta)$, where the second pseudoscalar term $\left(\alpha_{\mu} \vec{P} \cdot k=a \cos \theta\right)$ is due to non conservation of parity. In the Lederman et al. experiment [18] a large asymmetry of $e^{+}$was found $\left(a \simeq-\frac{1}{2}\right)$.

Let us discuss the Hamiltonian of the $\beta$-decay. The Hamiltonian (11) is a scalar. It conserves the parity. In order to take into account the results of the $\mathrm{Wu}$ et al. and other experiments we must assume that the Hamiltonian of the $\beta$-decay is the sum of a scalar and a pseudoscalar. In order to build such a Hamiltonian we have to add to five scalars which enter into the Hamiltonian (11) additional five pseudoscalars which are formed from products of the scalar $\bar{p}(x) n(x)$ and pseudoscalar $\bar{e}(x) \gamma_{5} \nu(x)$, vector $\bar{p}(x) \gamma^{\alpha} n(x)$ and pseudovector $\bar{e}(x) \gamma^{\alpha} \gamma_{5} \nu(x)$, etc. The most general Hamiltonian of the $\beta$-decay takes the form

$$
\mathcal{H}_{I}^{\beta}(x)=\sum_{i=S, V, T, A, P} \bar{p}(x) O_{i} n(x) \bar{e}(x) O^{i}\left(G_{i}+G_{i}^{\prime} \gamma_{5}\right) \nu(x)+\text { h.c. },
$$

where the constants $G_{i}$ characterize the scalar part of the Hamiltonian, the constants $G_{i}^{\prime}$ characterize the pseudoscalar part and the matrices $O^{i}$ are given by (12).

The Hamiltonian (37) is characterized by 10 (!) fundamental interaction constants. From the $\mathrm{Wu}$ et al. experiment it followed that scalar and pseudoscalar terms of the Hamiltonian must be of the same order. This means that the constants $\left|G_{i}\right|$ and $\left|G_{i}^{\prime}\right|$ (at least for some $i$ ) must be of the same order.

\footnotetext{
${ }^{16}$ Muon possesses longitudinal polarization if the probabilities of the emission of the muon with positive and negative helicities are different. This could happen only in the case if parity in the decay (35) is violated.
} 
In 1957-58 enormous progress in the development of the theory of the $\beta$-decay and other weak processes was reached. Soon after the discovery of the violation of parity the Hamiltonian of the weak interaction took a simple form, compatible with the experimental data.

The new development of the theory of the weak interaction started with the two-component theory of the neutrino.

\section{Massless two-component neutrino}

\section{Introduction}

The discovery of the violation of the parity in the $\beta$-decay and other weak processes triggered enormous progress in the understanding of the weak interaction. This progress started with the theory of the two-component neutrino. This theory of the neutrino became part of the universal $V-A$ theory of the weak interaction and the unified theory of the electromagnetic and weak interaction (Standard Model). The main idea of the two-component theory (left-handed component of the neutrino field in the interaction Hamiltonian) was generalized in the subsequent development of the theory of the weak interaction.

The two-component theory was based on the assumption that the neutrino is a massless particle. We know today that neutrinos have small but different from zero masses and that the two-component theory must be generalized.

Soon after the discovery of the parity violation Landau [19], Lee and Yang [20] and Salam [21] came to an idea of a possible connection of the violation of parity observed in the $\beta$-decay and other weak processes with neutrinos.

The neutrino field $\nu(x)$ satisfies the Dirac equation

$$
\left(i \gamma^{\alpha} \partial_{\alpha}-m_{\nu}\right) \nu(x)=0
$$

where $m_{\nu}$ is the neutrino mass.

Let us present the field $\nu(x)$ in the form of the sum of the left-handed $\nu_{L}(x)=\left(\frac{1-\gamma_{5}}{2}\right) \nu(x)$ and the right-handed $\nu_{L}(x)=\left(\frac{1+\gamma_{5}}{2}\right) \nu(x)$ components:

$$
\nu(x)=\nu_{L}(x)+\nu_{R}(x) .
$$


From (38) and (39) we obtain two coupled equations for $\nu_{L}(x)$ and $\nu_{R}(x)$

$$
i \gamma^{\alpha} \partial_{\alpha} \nu_{L}(x)-m_{\nu} \nu_{R}(x)=0 \quad i \gamma^{\alpha} \partial_{\alpha} \nu_{R}(x)-m_{\nu} \nu_{L}(x)=0 .
$$

Let us assume that $m_{\nu}=0$. In this case the equations for $\nu_{L}(x)$ and $\nu_{R}(x)$ are decoupled and we have

$$
i \gamma^{\alpha} \partial_{\alpha} \nu_{L, R}(x)=0 .
$$

Thus, for $m_{\nu}=0$, the neutrino field can be $\nu_{L}(x)$ (or $\nu_{R}(x)$ ). Such a theory can be valid only if parity is violated. In fact, under the inversion of coordinates the field $\nu(x)$ is transformed as follows:

$$
\nu^{\prime}\left(x^{\prime}\right)=\eta \gamma^{0} \nu(x) .
$$

Here $x^{\prime}=\left(x^{0}-\vec{x}\right)$ and $\eta$ is a phase factor. From (42) we have

$$
\nu_{L(R)}^{\prime}\left(x^{\prime}\right)=\eta \gamma^{0} \nu_{R(L)}(x) .
$$

Thus, under the inversion a left-handed (right-handed ) component of the field is transformed into a (right-handed) (left-handed) component. This means that equations (41) are not invariant under the inversion 17

From the investigation of the high-energy part of the tritium $\beta$-spectrum it was found only an upper bound for the neutrino mass which was much smaller than the mass of the electron, a particle emitted in the $\beta$-decay together with the neutrino. In 1957 Landau [19], Lee and Yang [20] and Salam [21] assumed that the neutrino mass was equal to zero and that the neutrino field is $\nu_{L}(x)$ (or $\nu_{R}(x)$ ). For reasons, which will be clear later, this theory is called the two-component neutrino theory.

There were two major consequences of the two-component neutrino theory.

1. Parity is strongly violated in the $\beta$-decay and in other processes in which neutrino(s) participate.

The most general Hamiltonian of the $\beta$-decay in the case of parity violation is given by expression (37). Five interaction constants $G_{i}$

\footnotetext{
${ }^{17}$ Equations (41) for massless spin $1 / 2$ particle were considered by $\mathrm{H}$. Weil in 1929 22. However, as they did not conserve parity they were rejected. In [23, Pauli wrote "...because the equation for $\nu_{L}(x)\left(\nu_{R}(x)\right)$ is not invariant under space reflection it is not applicable to the physical reality".
} 
characterize the scalar part of the Hamiltonian and five interaction constants $G_{i}^{\prime}$ characterize the pseudoscalar part $(i=S, V, T, A, P)$.

In the case of the two-component theory these constants are connected by the relations

$$
G_{i}^{\prime}=-G_{i} \quad\left(\text { if neutrino field is } \nu_{\mathrm{L}}(\mathrm{x})\right)
$$

and

$$
\left.G_{i}^{\prime}=G_{i} \quad \text { (if neutrino field is } \nu_{\mathrm{R}}(\mathrm{x})\right) .
$$

The most general Hamiltonian of the $\beta$-decay takes the form

$$
\mathcal{H}_{I}^{\beta}(x)=\sum_{i=S, V, T, A, P} G_{i} \bar{p}(x) O_{i} n(x) \bar{e}(x) O^{i}\left(1 \mp \gamma_{5}\right) \nu(x)+\text { h.c.. }
$$

From this expression it follows that effects of violation of parity in the $\beta$-decay will be large (maximal).

2. The neutrino helicity (projection of the spin onto the direction of momentum) is equal to $-1(+1)$ in the case if the neutrino field is $\nu_{L}(x)$ $\left(\nu_{R}(x)\right) 18$

The theory we are discussing is called the two-component neutrino theory by the following reason. In the general case of a Dirac particle with spin $1 / 2$ there are four states with momentum $\vec{p}$ and energy $E_{p}=\sqrt{p^{2}+m^{2}}$ : two particle states with helicities \pm 1 and two antiparticle states with helicities \pm 1 . In the two-component theory with the neutrino field $\nu_{L}(x)\left(\nu_{R}(x)\right)$ only the state of the neutrino with helicity $-1(+1)$ and the state of the antineutrino with helicity $+1(-1)$ are allowed.

It is easy to see that in the processes in which a two-component neutrino is emitted large (maximal) violation of parity will be observed. In fact, let $w_{r}^{R}$ be the probability to emit a neutrino with helicity $r$ in a right-handed

\footnotetext{
${ }^{18}$ The spinor $u^{r}(p)$ which describes a massless neutrino with momentum $p$ and helicity $r$ satisfies the equations $\gamma \cdot p u^{r}(p)=\left(\gamma^{0} p^{0}-\vec{\gamma} \vec{p}\right) u^{r}(p)=0, \quad \vec{\Sigma} \cdot \vec{k} u^{r}(p)=r u^{r}(p)$. Here $\vec{\Sigma}=\gamma_{5} \gamma^{0} \vec{\gamma}$ is the spin operator and $\vec{k}$ is the unit vector in the direction of the momentum $\vec{p}$. From these equations we find $\gamma_{5} u^{r}(p)=r u^{r}(p)$. In $\nu_{L}(x)\left(\nu_{R}(x)\right)$ the spinor $u^{r}(p)$ is multiplied by the projection operator $\frac{1-\gamma_{5}}{2}\left(\frac{1-\gamma_{5}}{2}\right)$. We have $\frac{1-\gamma_{5}}{2} u^{r}(p)=\frac{1-r}{2} u^{r}(p)$ $\left(\frac{1+\gamma_{5}}{2} u^{r}(p)=\frac{1+r}{2} u^{r}(p)\right)$. Thus, $r=-1(r=1)$ in the case that the neutrino field is $\nu_{L}(x)\left(\nu_{R}(x)\right)$.
} 
system. This probability is equal to the probability of the emission of a neutrino with helicity $-r$ in a left-handed system

$$
w_{r}^{R}=w_{-r}^{L} .
$$

If the parity is conserved

$$
w_{r}^{R}=w_{r}^{L} .
$$

From (47) and (48) it follows that in the case of the conservation of parity the probabilities of the emission of neutrinos with helicities $r$ and $-r$ must be equal

$$
w_{r}^{L, R}=w_{-r}^{L, R}, \quad \text { i. e. } \quad w_{1}^{L, R}=w_{-1}^{L, R}
$$

In the case of the two-component neutrino theory $w_{1}=0\left(\right.$ or $\left.w_{-1}=0\right)$. Thus, in the two-component theory relation (49) is maximally violated.

We will discuss now the difference between a neutrino and an antineutrino. In general particles and antiparticles have the same mass but different (in sign) charges. There exist in nature different conserved charges. The most familiar is the electric charge $Q$. The electric charges of the proton, neutron, electron, neutrino, for example, are equal to $1,0,-1,0$, respectively 19

Another conserved charge is the baryon number $B$. The baryon numbers of the proton, neutron, electron, neutrino are equal to 1,1,0,0, respectively.

We will be interested here mainly in the lepton number $L$. The lepton numbers of the proton, neutron, electron, neutrino are defined as $0,0,1,1$, respectively.

Particles like a proton and a neutron which possess a baryon number are called baryons. An electron, a neutrino and other particles which possess the lepton number are called leptons.

According to the Quantum Field Theory, the existence of a particle with a mass $m$ and some charges implies the existence of an antiparticle which has the same mass $m$ and opposite charges. This general consequence of the Quantum Field Theory was confirmed by numerous experiments. For example, the antiparticles of the proton $p$ and the neutron $n$ are the antiproton $\bar{p}$ $(Q=-1, B=-1, L=0)$ and the antineutron $\bar{n}(Q=0, B=-1, L=0)$. The positron $e^{+}$is the antiparticle of the electron. Its mass is equal to $m_{e}, Q=1, B=0, L=-1$. The antiparticle of the neutrino is the antineutrino $\bar{\nu}$. It has $Q=0, B=0, L=-1$.

\footnotetext{
${ }^{19}$ Usually, charges of particles are expressed in the unit of the proton charge.
} 
Due to the conservation of the lepton number, for example, in the $\beta$-decay of the neutron an electron and an antineutrino are emitted

$$
n \rightarrow p+e^{-}+\bar{\nu}
$$

There exist in nature also neutral particles with all charges equal to zero. Examples are the $\gamma$-quantum, the $\pi^{0}$-meson, etc. In the case of such particles there is no notion of antiparticles (or particles and antiparticles are identical).

In 1937 [24 great Italian physicist E. Majorana proposed a theory of truly neutral particles with spin equal to $1 / 2$ (which today are called Majorana particles). E. Majorana was not satisfied with the existing at that time theory of electrons and positrons in which positrons were considered as holes in the Dirac sea of the states of electrons with negative energies. He wanted to formulate the symmetrical theory in which there is no notion of states with negative energies. In the paper "Symmetrical theory of electron and positron" [24] he came also to a theory of spin 1/2 particles in which particles and antiparticles are identical. Majorana wrote in the paper [24]: "A generalization of Jordan-Wigner quantization method allows not only to give symmetrical form to the electron-positron theory but also to construct an essentially new theory for particles without electric charge (neutrons and hypothetical neutrinos)".

It is an open problem if the neutrino is a truly neutral Majorana particle or a Dirac particle which possesses a lepton number. This is one of the most important problems of modern neutrino physics and we will discuss it later. Now we notice that if the lepton number is conserved, the neutrino is a Dirac particle and $L(\nu)=-L(\bar{\nu})=1$. If there is no conserved lepton number, the neutrino is a truly neutral Majorana particle. In the case of the Majorana neutrino with $m \neq 0$ there are only two states with momentum $\vec{p}$ and energy $E_{p}=\sqrt{p^{2}+m^{2}}$ : states with helicities \pm 1 . Let us notice that for the massless neutrino the theory of the two-component Dirac neutrino and the Majorana neutrino are equivalent.

Before finishing this section we would like to notice that Landau [19], Lee and Yang [20] and Salam [21] had different arguments in favor of the two-component neutrino theory.

Landau assumed that the neutrino mass was equal to zero and for the neutrino field he chose $\nu_{L}(x)$ (or $\nu_{R}(x)$ ) assuming $C P$ invariance of the weak interaction ( $C$ is charge conjugation, i.e. the operation of transition from particles to antiparticles). Lee and Yang assumed that the neutrino is a 
particle with helicity equal to -1 (or +1 ). This is possible only if the neutrino mass is equal to zero, parity is violated and the neutrino field is $\nu_{L}(x)$ (or $\left.\nu_{R}(x)\right)$. Salam assumed invariance of the equation for the neutrino field under $\gamma_{5}$-transformation $\left(\nu \rightarrow \gamma_{5} \nu\right)$. From this requirement it follows that the neutrino mass is equal to zero and the neutrino field is $\nu_{L}(x)\left(\right.$ or $\left.\nu_{R}(x)\right)$.

\section{Measurement of neutrino helicity. Gold- haber et al. experiment}

Soon after the two-component neutrino theory had been proposed, the neutrino helicity was determined from the results of the spectacular Goldhaber, Grodzins and Sunyar experiment [25].

In this experiment the neutrino helicity was obtained from the measurement of the circular polarization of $\gamma$ 's produced in the chain of reactions

$$
\begin{aligned}
e^{-}+{ }^{152} \mathrm{Eu} \rightarrow \nu+\quad & { }^{152} \mathrm{Sm}^{*} \\
& \downarrow \\
& { }^{152} \mathrm{Sm}+\gamma .
\end{aligned}
$$

The spins of ${ }^{152} \mathrm{Eu}$ and ${ }^{152} \mathrm{Sm}$ are equal to zero and the spin of ${ }^{152} \mathrm{Sm}^{*}$ is equal to one. Since the orbital momentum of the initial electron is equal to zero (K-capture), from the conservation of the projection of the total angular momentum on the neutrino momentum we have

$$
\frac{1}{2} h+m= \pm \frac{1}{2}
$$

where $h$ is the neutrino helicity and $m$ is the projection of the spin of ${ }^{152} \mathrm{Sm}^{*}$. From this relation we have

$$
m=0,-1 \text { for } h=1, \quad m=0,+1 \text { for } h=-1 .
$$

Thus, the circular polarization of $\gamma^{\prime}$ s emitted in the direction of the ${ }^{152} \mathrm{Sm}^{*}$ momentum is equal to the helicity of the neutrino. In the Goldhaber et al. experiment, the circular polarization of resonantly scattered $\gamma$ 's was measured (only $\gamma$ 's emitted in the direction of motion of ${ }^{152} \mathrm{Sm}^{*}$ satisfy the resonance condition). The authors concluded "... our result is compatible with $100 \%$ negative helicity of neutrino emitted in orbital electron capture".

Thus, the Goldhaber et al. experiment confirmed the two-component neutrino theory. It was established that from the two possibilities for the neutrino field $\left(\nu_{L}(x)\right.$ or $\left.\nu_{R}(x)\right)$ the first possibility was realized. 


\section{Universal current $\times$ current $\mathrm{V}$-A theory}

\section{Introduction}

The $V-A$ current $\times$ current theory of the weak interaction signified a great progress in the understanding of the weak interaction and neutrino. The Feynman and Gell-Mann, Marshak and Sudarshan idea of the left-handed components of all fields in the CC Hamiltonian was triggered mainly by some experimental data, success of the two-component neutrino theory and great intuition. The idea of the left-handed components complemented with the idea of the universality of the weak interaction made it possible to build the simplest possible CC Hamiltonian of the weak interaction which is characterized by only one (Fermi) constant. The authors of this theory were courageous enough to state that some experimental data which existed at that time but contradicted this theory were wrong. Further experiments showed that the authors were correct: current $\times$ current $V-A$ theory is in perfect agreement with all existing CC data.

The most general Hamiltonian of the $\beta$-decay in the case of the twocomponent neutrino is given by expression (46). It includes five terms (S, V,T,A,P). There were many attempts to determine the dominant terms of the Hamiltonian from the data of different $\beta$-decay experiments. However, during many years the situation was contradictory. From the measurement of the angular electron-neutrino correlation in the decay ${ }^{6} \mathrm{He} \rightarrow{ }^{6} \mathrm{Li}+e^{-}+\bar{\nu}$ and from other data it followed that S,T terms must be the dominant ones. On the other hand, the data on the measurement of electron-neutrino correlation in the decay ${ }^{35} \mathrm{Ar} \rightarrow{ }^{35} \mathrm{Cl}+e^{+}+\nu$ and other data were in favor of $\mathrm{V}, \mathrm{A}$ terms.

In this uncertain experimental situation in 1958 two fundamental theoretical papers by Feynman and Gell-Mann [26] and Marshak and Sudarshan [27] appeared. These authors proposed a principle which allowed them to build the simplest possible universal theory of the $\beta$-decay and other weak processes.

This theory was a generalization of the two-component neutrino theory. Feynman and Gell-Mann, Marshak and Sudarshan assumed that in the Hamiltonian of the weak interaction there enter not only the left-handed component of the massless neutrino field but left-handed components of all fermion fields. Thus, Feynman and Gell-Mann, Marshak and Sudarshan assumed that there has no connection between the mass of a particle and the 
fact that the left-handed component of its field enters into the Hamiltonian of the weak interaction. Feynman and Gell-Mann assumed that $\left(\frac{1-\gamma_{5}}{2}\right) \psi_{a}(x)$ enters into the Hamiltonian of the weak interaction because this field satisfies a second order equation and could be considered as a fundamental field. Marshak and Sudarshan came to left-handed components from the requirement of $\gamma_{5}$ invariance of the interaction (invariance under the change $\left.\psi_{a}(x) \rightarrow-\gamma_{5} \psi_{a}(x)\right)$.

The Hamiltonian of the $\beta$-decay has in this case the form

$$
\mathcal{H}_{I}^{\beta}(x)=\sum_{i=S, V, T, A, P} G_{i} \bar{p}_{L}(x) O_{i} n_{L}(x) \bar{e}_{L}(x) O^{i} \nu_{L}(x)+\text { h.c.. }
$$

We have

$$
\bar{p}_{L}(x) O_{i} n_{L}(x)=\bar{p}(x) \frac{1+\gamma_{5}}{2} O_{i} \frac{1-\gamma_{5}}{2} n(x) .
$$

Using the algebra of the Dirac matrices $\gamma$ 's it is easy to show that

$$
\frac{1+\gamma_{5}}{2}\left(1 ; \sigma_{\alpha \beta} ; \gamma_{5}\right) \frac{1-\gamma_{5}}{2}=0
$$

Thus, $S, T$ and $P$ terms do not enter into the Hamiltonian (53). Moreover, $A$ and $V$ terms are connected by the relation:

$$
\frac{1+\gamma_{5}}{2} \gamma_{\alpha} \gamma_{5} \frac{1-\gamma_{5}}{2}=-\frac{1+\gamma_{5}}{2} \gamma_{\alpha} \frac{1-\gamma_{5}}{2}
$$

The Hamiltonian of the $\beta$-decay takes the simplest possible form

$$
\begin{aligned}
\mathcal{H}_{I}^{\beta}(x) & =\frac{G_{F}}{\sqrt{2}} 4 \bar{p}_{L}(x) \gamma_{\alpha} n_{L}(x) \bar{e}_{L}(x) \gamma^{\alpha} \nu_{L}(x)+\text { h.c. } \\
& =\frac{G_{F}}{\sqrt{2}} \bar{p}(x) \gamma_{\alpha}\left(1-\gamma_{5}\right) n(x) \bar{e}(x) \gamma^{\alpha}\left(1-\gamma_{5}\right) \nu(x)+\text { h.c.. }
\end{aligned}
$$

The Hamiltonian (56), like the Fermi Hamiltonian(4), is characterized by only one interaction constant $G_{F} .20$ There is, however, a crucial difference between the Hamiltonian (56) and the Fermi Hamiltonian. In the Hamiltonian (56) left-handed components of all fields enter. This means that the Hamiltonian (56) unlike the Fermi Hamiltonian does not conserve parity.

\footnotetext{
${ }^{20}$ Interesting that the title of the Feynman and Gell-Mann paper is "Theory of the Fermi interaction".
} 
What about numerous experiments from which it followed that $\mathrm{S}$ and $\mathrm{T}$ terms are the dominant terms of the Hamiltonian of the $\beta$-decay? In the Feynman and Gell-Mann paper it is written "These theoretical arguments seem to the authors to be strong enough to suggest that the disagreement with ${ }^{6} \mathrm{He}$ recoil experiment and with some other less accurate experiments indicates that these experiments are wrong". In fact, subsequent experiments did not confirm the results of the experiments which indicated in favor of the dominance of $\mathrm{S}$ and $\mathrm{T}$ terms. The Hamiltonian (56) described data of all experiments concerning the study of the $\beta$-decay.

With the Feynman-Gell-Mann, Marshak-Sudarshan prescription (left-handed components of all fields enter into the Hamiltonian of the weak interaction), which lead to the unique expression for the interaction Hamiltonian, it was natural to implement the idea of the universal weak interaction which we discussed before.

For the Hamiltonian of the decay

$$
\mu^{+} \rightarrow e^{+}+\nu+\bar{\nu}
$$

we have in this case

$$
\mathcal{H}_{I}^{\mu \rightarrow e \nu \bar{\nu}}(x)=\frac{G_{F}}{\sqrt{2}} 4 \bar{e}_{L}(x) \gamma_{\alpha} \nu_{L}(x) \bar{\nu}_{L}(x) \gamma^{\alpha} \mu_{L}(x)+\text { h.c. }
$$

From (58) it follows that the lifetime of the muon is given by the expression $\tau_{\mu}=\frac{192 \pi^{3}}{G_{F}^{2} m_{\mu}^{5}}$, where $m_{\mu}$ is the mass of the muon. Feynman and Gell-Mann demonstrated that if we take for $G_{F}$ the value obtained from the superallowed $0^{+} \rightarrow 0^{+} \beta$-decay of ${ }^{14} \mathrm{O}$, we will find perfect agreement with the experimental lifetime. This was an important confirmation of the hypothesis of the universality of the weak interaction.

This agreement was also an evidence in favor of the conserved vector current (CVC) hypothesis [28]. According to this hypothesis the weak vector current is the "charged" component of the isovector current which is conserved due to isotopic invariance. The conservation of the vector current ensures the fact that the Fermi constant is not renormalized by the strong interaction.

From the $\mu-e$ universality it followed that the Hamiltonian of the $\mu$ capture and other connected processes can be obtained from (56) by the change $e(x) \rightarrow \mu(x)$. We have

$$
\mathcal{H}_{I}^{\mu}(x)=\frac{G_{F}}{\sqrt{2}} 4 \bar{p}_{L}(x) \gamma_{\alpha} n_{L}(x) \bar{\mu}_{L}(x) \gamma^{\alpha} \nu_{L}(x)+\text { h.c. }
$$


At the time when Feynman and Gell-Mann, Marshak and Sudarshan wrote their papers there was a contradiction to the idea of $\mu-e$ universality of the weak interaction with the data on the measurement of the width of the decay $\pi^{+} \rightarrow e^{+}+\nu$. From (56) and (59) it follows that the ratio of the decay widths $R=\frac{\Gamma\left(\pi^{+} \rightarrow e^{+} \nu\right)}{\Gamma\left(\pi^{+} \rightarrow \mu^{+} \nu\right)}$ is given by the expression

$$
R=\frac{m_{e}^{2}}{m_{\mu}^{2}} \frac{\left(1-\frac{m_{e}^{2}}{m_{\pi}^{2}}\right)^{2}}{\left(1-\frac{m_{\mu}^{2}}{m_{\pi}^{2}}\right)^{2}} \simeq 1.2 \cdot 10^{-4} .
$$

On the other hand in the experiment [29] the decay $\pi^{+} \rightarrow e^{+}+\nu$ was not observed and it was found $R<10^{-5}$. Feynman and Gell-Mann wrote "This is a very serious discrepancy. The authors have no idea on how it can be resolved".

In 1958 a new experiment on measurement of the $\pi^{+} \rightarrow e^{+}+\nu$-decay was performed at CERN [30]. In this experiment, perfect agreement with prediction (60) of the universal Feynman and Gell-Mann, Marshak and Sudarshan theory was obtained 21

In order to unify interactions (56), (58) and (59) Feynman and Gell-Mann introduced the $\mu-e$ symmetric weak current

$$
j^{\alpha}=2\left(\bar{p}_{L} \gamma^{\alpha} n_{L}+\bar{\nu}_{L} \gamma^{\alpha} e_{L}+\bar{\nu}_{L} \gamma^{\alpha} \mu_{L}\right)
$$

and assumed that the total Hamiltonian of the weak interaction had the current $\times$ current form

$$
\mathcal{H}_{I}=\frac{G_{F}}{\sqrt{2}} j^{\alpha} j_{\alpha}^{+}
$$

where $G_{F}$ was the Fermi constant. Two remarks are in order.

1. The hadron part of the current has the form

$$
j^{\alpha}=v^{\alpha}-a^{\alpha}
$$

where $v^{\alpha}=\bar{p} \gamma^{\alpha} n$ and $a^{\alpha}=\bar{p} \gamma^{\alpha} \gamma_{5} n$ are the vector and axial currents 22 Notice that Fermi $\beta$-transitions of nuclei are due to the vector current and Gamov-Teller transitions are due to the axial current.

\footnotetext{
${ }^{21}$ When this result was obtained Feynman was visiting CERN. The news reached him when he was queuing in the CERN cafeteria. It is said that when Feynman learnt about the $\pi \rightarrow e \nu$-news he started to dance.

${ }^{22}$ This is the reason why the Feynman and Gell-Mann, Marshak and Sudarshan theory is called the $V-A$ theory.
} 
2. The current $j^{\alpha}$ provides transitions $n \rightarrow p, e^{-} \rightarrow \nu$, etc. in which $\Delta Q=Q_{f}-Q_{i}=1\left(Q_{i}\left(Q_{f}\right)\right.$ is the initial (final) charge). By this reason the current $j^{\alpha}$ is called the charged current $(\mathrm{CC})$.

There are two types of terms in the Hamiltonian (62): nondiagonal and diagonal. The nondiagonal terms have the form

$$
\begin{array}{r}
\mathcal{H}_{I}^{n d}=\frac{G_{F}}{\sqrt{2}} 4\left\{\left[\left(\bar{p}_{L} \gamma^{\alpha} n_{L}\right)\left(\bar{e}_{L} \gamma_{\alpha} \nu_{L}\right)+\text { h.c. }\right]+\right. \\
{\left[\left(\bar{p}_{L} \gamma^{\alpha} n_{L}\right)\left(\bar{\mu}_{L} \gamma_{\alpha} \nu_{L}\right)+\text { h.c. }\right]+} \\
\left.\left[\left(\bar{e}_{L} \gamma^{\alpha} \nu_{L}\right)\left(\bar{\nu}_{L} \gamma_{\alpha} \mu_{L}\right)+\text { h.c. }\right]\right\}
\end{array}
$$

The first term of this expression is the Hamiltonian of $\beta$-decay of the neutron $n \rightarrow p+e^{-}+\bar{\nu}$, of the process $\bar{\nu}+p \rightarrow e^{+}+n$ and other processes. The second term of (63) is the Hamiltonian of the process $\mu^{-}+p \rightarrow \nu+n$, of the neutrino process $\nu+n \rightarrow \mu^{-}+p$ and other processes. Finally, the third term of (63) is the Hamiltonian of the $\mu$-decay (57), of the process $\nu+e^{-} \rightarrow \mu^{-}+\nu$ and other processes.

The diagonal terms of the Hamiltonian (62) are given by

$$
\mathcal{H}^{d}=\frac{G_{\mathrm{F}}}{\sqrt{2}} 4\left[\left(\bar{\nu}_{L} \gamma^{\alpha} e_{L}\right)\left(\bar{e}_{L} \gamma_{\alpha} \nu_{L}\right)+\left(\bar{\nu}_{L} \gamma^{\alpha} \mu_{L}\right)\left(\bar{\mu}_{L} \gamma_{\alpha} \nu_{L}\right)+\left(\bar{p}_{L} \gamma^{\alpha} n_{L}\right)\left(\bar{n}_{L} \gamma_{\alpha} p_{L}\right)\right]
$$

The first term of (64) is the Hamiltonian of the processes of elastic scattering of a neutrino and an antineutrino on an electron

$$
\nu+e \rightarrow \nu+e
$$

and

$$
\bar{\nu}+e \rightarrow \bar{\nu}+e,
$$

of the process $e^{+}+e^{-} \rightarrow \bar{\nu}+\nu$ and other processes. Such processes were not known in the fifties. Their existence and the cross sections of these processes were predicted by the current $\times$ current theory.

The cross sections of the processes (65) and (66) are very small (at MeV's energies of the order $\left.10^{-45} \mathrm{~cm}^{2}\right)$. The observation of such processes was a challenge. After many years of efforts, the cross section of the process (66) was measured by F. Reines et al. [31] in an experiment with antineutrinos from a reactor. At that time, the Glashow-Weinberg-Salam Standard Model already existed. According to the Standard model, to the matrix elements 
of the processes (65) and (66) there contribute the Hamiltonian (64) and an additional (so called neutral current) Hamiltonian. The result of the experiment by F.Reines et al. was in agreement with the Standard Model.

In the Feynman and Gell-Mann and Marshak and Sudarshan papers decays of the $\Lambda$-hyperon and other strange particles were also briefly discussed. However, weak interaction of the strange particles was fully included into the current $\times$ current Hamiltonian in 1963 by N. Cabibbo [32. We will discuss Cabibbo's contribution to the theory of weak interaction later.

\section{Intermediate vector $W$ boson}

\section{Introduction}

The great Yukawa idea that the interaction between nucleons is due to the exchange of a meson (which allowed him to predict the $\pi$-meson) was applied by Klein to the short range weak interaction. Klein assumed that the weak decay of the neutron was due to the exchange of a heavy charged vector boson between $(n p)$ and $(e \nu)$ pairs. It is impressive that this general quantum idea very early in the thirties allowed one to anticipate the existence of a very heavy particle which could be observed only many years later after modern high-energy accelerators were built.

In the Feynman and Gell-Mann paper, which we discussed in the previous section, it was mentioned that the current $\times$ current Hamiltonian of the weak interaction (62) could originate from the exchange of a heavy intermediate charged vector meson.( "We have adopted the point of view that the weak interactions all arise from the interaction of a current $J_{\alpha}$ with itself, possibly via an intermediate charged vector meson of high mass" [26].) We will discuss now the hypothesis of a charged intermediate vector boson. Let us assume that there exists a charged vector $W^{ \pm}$boson and that the Lagrangian of the weak interaction has the form of a scalar product of the current $j^{\alpha}$ given by Eq.(61) and the vector field $W_{\alpha}$

$$
\mathcal{L}_{\mathcal{I}}=-\frac{g}{2 \sqrt{2}} j_{\alpha} W^{\alpha}+\text { h.c. }
$$

where $g$ is a dimensionless interaction constant. 
Let us notice that the Lagrangian (67) has the form analogous to the Lagrangian of the electromagnetic interaction

$$
\mathcal{L}_{I}^{E M}=-e j_{\alpha}^{E M} A^{\alpha} .
$$

where $j_{\alpha}^{E M}$ is the electromagnetic current, $A^{\alpha}$ is the electromagnetic field and $e$ is the electric charge.

If the Lagrangian of the weak interaction has the form (67), in this case the $\beta$-decay of the neutron proceeds in the following three steps: 1 . neutron produces the virtual $W^{-}$-boson and is transferred into proton; 2 . the virtual $W^{-}$-boson propagates; 3 . the virtual $W^{-}$-boson decays into a electron and an antineutrino. In the Feynman diagram, the propagator of the $W$-boson contains a factor $\frac{-1}{Q^{2}-m_{W}^{2}}$, where $Q=p_{n}-p_{p}$ is the momentum transfer and $m_{W}$ is the mass of the $W$-boson. If the $W$-boson is a heavy particle (say, with a mass which is much larger than the mass of the proton), in this case $Q^{2}$ in the $W$-propagator can be safely neglected and the matrix element of the $\beta$-decay of the neutron can be obtained from the Hamiltonian (62) in which the Fermi constant is given by the relation

$$
\frac{G_{\mathrm{F}}}{\sqrt{2}}=\frac{g^{2}}{8 m_{W}^{2}} .
$$

In a similar way it can be shown that in the region of relatively small energies, the matrix elements of all weak processes with the virtual (intermediate) charged $W$-boson can be obtained from the current $\times$ current Hamiltonian (62) in which the Fermi constant is given by relation (69) 23

Thus, the theory with the vector $W^{ \pm}$-boson could explain the current $\times$current structure of the weak interaction Hamiltonian and the fact that the Fermi constant has the dimension $[M]^{-2}$.

We know today that the intermediate charged $W^{ \pm}$-boson exists. The $W^{ \pm}$boson is one of the heaviest particles: its mass is equal to $m_{W} \simeq 80.4 \mathrm{GeV}$. For the discovery of the $W^{ \pm}$-boson and the $Z^{0}$-boson (see later) in $1984 \mathrm{C}$. Rubbia and S. van der Meer were awarded the Nobel Prize. As we will see later, the Lagrangian (67) is part of the total Lagrangian of the Standard Model.

The first idea of the charged vector boson, mediator of the weak interaction, was discussed by O. Klein [33] in 1938, soon after the Fermi $\beta$-decay

\footnotetext{
${ }^{23}$ From the point of view of the theory with the $W$-boson, the current $\times$ current Hamiltonian with the Fermi constant (69) is the effective Hamiltonian of the weak interaction.
} 
paper had appeared. Fermi built the first Hamiltonian of the $\beta$-decay by analogy with electrodynamics. O.Klein noticed that the analogy would be more complete if a charged vector boson (analog of the $\gamma$-quantum) existed and the weak interaction originated from an interaction which (like the electromagnetic interaction) had the form of a product of a current and a vector field. In order to build such a theory O.Klein assumed gauge invariance.

\section{The first method of neutrino detection}

As we discussed before, because of the extreme smallness of the cross section for the absorption of neutrinos by nuclei for many years most physicists considered the neutrino as an undetectable particle.

The first method of neutrino detection was proposed by B. Pontecorvo in 1946 [10]. He wrote "The object of this note is to show that the experimental observation of an inverse $\beta$ process produced by neutrino is not out of the question with the modern experimental facilities, and to suggest a method which might make an experimental observation feasible."

Pontecorvo proposed radiochemical methods of neutrino detection. Let us consider, as an example, the reaction

$$
\nu+{ }^{37} \mathrm{Cl} \rightarrow e^{-}+{ }^{37} \mathrm{Ar} .
$$

The ${ }^{37} \mathrm{Ar}$ atoms decay (via K-capture) with a life-time of about 34 days.

After irradiation of a target (containing ${ }^{37} \mathrm{Cl}$ ) by neutrinos for a relatively long time (say, one month) a few radioactive atoms of ${ }^{37} \mathrm{Ar}$ could be produced. As argon is a nobel gas, atoms of ${ }^{37} \mathrm{Ar}$ can be extracted from the target and can be placed into a proportional counter in which their decay will be detected. This is the main idea of Pontecorvo's radiochemical method. He discussed different reactions. He considered the $\mathrm{Cl}$-Ar reaction (70) as very appropriate for the neutrino detection (a large volume of liquid Carbon Tetra-Chloride can be used as a target, ${ }^{37} \mathrm{Ar}$ atoms have a convenient lifetime, etc.).

In the report [10] B.Pontecorvo also pointed out the following intensive sources of neutrinos which existed at that time:

1. The Sun. The flux of the solar neutrinos is equal to $6 \cdot 10^{10} \frac{\nu}{\mathrm{cm}^{2} \mathrm{sec}}$ 
2. Nuclear reactors during operation. 24 The total flux of (anti) neutrinos from a reactor is approximately equal to $2 \times 10^{20} \frac{\bar{\nu}}{\mathrm{sec}}$ per $G W_{\text {thermal }}$.

3. Radioactive sources which can be prepared in reactors.

Pontecorvo's radiochemical method of neutrino detection was used in solar neutrino experiments. The first experiment in which solar neutrinos were detected was performed by R. Davis and collaborators [11]. In this experiment solar neutrinos were detected via the observation of the $\mathrm{Cl}-\mathrm{Ar}$ reaction (70). In 2002, R. Davis was awarded the Nobel Prize for this experiment.

\section{Detection of neutrino. Reines and Cowan experiment}

\section{Introduction}

The pioneering reactor neutrino experiment by Reines and Cowan proved the Pauli-Fermi hypothesis of the neutrino. The value of the cross section measured in this experiment confirmed the correctness of the V-A theory of weak interaction. This experiment opened a new era in neutrino physics: era of experiments with reactor neutrinos.

The first proof of the existence of neutrinos was obtained in 1953-59 in the F.Reines and C.L. Cowan experiments 34]. In these experiments antineutrinos from the Savannah River reactor 25 were detected through the observation of the process

$$
\bar{\nu}+p \rightarrow e^{+}+n
$$

Antineutrinos are produced in a reactor in a chain of $\beta$-decays of neutronrich nuclei, products of the fission of uranium and plutonium. The energies of antineutrinos from a reactor are $\lesssim 10 \mathrm{MeV}$. About $2.3 \cdot 10^{20}$ antineutrinos

\footnotetext{
${ }^{24}$ Pontecorvo wrote "Probably this is the most convenient neutrino source".

${ }^{25}$ In the beginning Reines and Cowan planned to do an experiment with neutrinos from an atomic bomb explosion. Later they understood that an experiment with reactor antineutrinos was simpler and feasible. Reines remembered in his Nobel lecture "I have wondered since why it took so long for us to come to this now obvious conclusion and how it escaped others around us with whom we talked..."
} 
per second were emitted by the Savannah River reactor. The flux of $\bar{\nu}_{e}$ 's in the Reines and Cowan experiment was about $10^{13} \mathrm{~cm}^{-2} \mathrm{~s}^{-1}$.

A liquid scintillator $26\left(1.4 \cdot 10^{3}\right.$ liters $)$ loaded with $\mathrm{CdCl}_{2}$ was used as a target in the experiment. A positron produced in the process (71), slowed down in the scintillator and annihilated with an electron, producing two $\gamma$ quanta with energies of $\simeq 0.51 \mathrm{MeV}$ and opposite momenta.

A neutron, produced in the process (71), slowed down in the target and was captured by $\mathrm{Cd}$ within about $5 \mu \mathrm{s}$, producing a $\gamma$-quantum in the process $n+{ }^{108} \mathrm{Cd} \rightarrow{ }^{109} \mathrm{Cd}+\gamma$ (at small energies the cross section of this process is very large). The $\gamma$-quanta were detected by 110 photomultipliers. Thus, the signature of the $\bar{\nu}$-event in the Reines and Cowan experiment was two $\gamma$-quanta from the $e^{+}-e^{-}$-annihilation in coincidence with a delayed $\gamma$ quantum from the neutron capture by cadmium. For the cross section of the process (171) the value

$$
\sigma_{\nu}=(11 \pm 2.6) 10^{-44} \mathrm{~cm}^{2}
$$

was obtained in the latest measurements [34]. This value was in agreement with the predicted value.

In the $\mathrm{V}$-A current $\times$ current theory the cross section of the process (71) is connected with the life-time $\tau_{n}$ of the neutron by the relation

$$
\sigma\left(\bar{\nu}_{e} p \rightarrow e^{+} n\right)=\frac{2 \pi^{2}}{m_{e}^{5} f \tau_{n}} p_{e} E_{e}
$$

where $E_{e} \simeq E_{\bar{\nu}}-\left(m_{n}-m_{p}\right)$ is the energy of the positron, $p_{e}$ is the positron momentum, $\mathrm{f}=1.686$ is the phase-space factor, $m_{n}, m_{p}, m_{e}$ are the masses of the neutron, proton and electron, respectively. From (73) for the cross section of the process (71), averaged over the antineutrino spectrum, the value

$$
\bar{\sigma}\left(\bar{\nu}_{e} p \rightarrow e^{+} n\right) \simeq 9.5 \cdot 10^{-44} \mathrm{~cm}^{2}
$$

was found. In 1995 the Nobel Prize in Physics was awarded to F. Reines "for the detection of the neutrino" 27

\footnotetext{
${ }^{26}$ Reines and Cowan were the first who understood that the phenomenon of scintillation of organic liquids, discovered at that time, could be employed in order to build a big (1 $m^{3}$ ) detector which was necessary to detect neutrinos.

${ }^{27}$ Clyde Cowan died in 1974.
} 


\section{Lepton number conservation. Davis ex- periment}

The particle which is produced in the $\beta$-decay together with the electron is called the antineutrino. It is a direct consequence of the quantum field theory that an antineutrino can produce a positron in the process (171) and other similar processes. Can antineutrinos produce electrons in weak-interaction processes with nucleons? This question was studied in an experiment which was performed in 1956 by Davis [35] at the Savannah River reactor. This was the first application of Pontecorvo's radiochemical method. Radioactive ${ }^{37} \mathrm{Ar}$ atoms which could be produced in the process

$$
\bar{\nu}+{ }^{37} \mathrm{Cl} \rightarrow \mathrm{e}^{-}+{ }^{37} \mathrm{Ar}
$$

were searched for in this experiment. No ${ }^{37} \mathrm{Ar}$ atoms were found. For the cross section of the process (75) the following upper bound was obtained:

$$
\sigma\left(\bar{\nu}+{ }^{37} \mathrm{Cl} \rightarrow \mathrm{e}^{-}+{ }^{37} \mathrm{Ar}\right)<0.9 \cdot 10^{-45} \mathrm{~cm}^{2} .
$$

This bound is about five times smaller than the cross section of the corresponding reaction with the neutrino.

Thus, it was established that antineutrinos from a reactor can produce positrons (the Reines-Cowan experiment) but can not produce electrons (the Davis experiment).

This result can be explained if we assume that there exists conserving lepton number $L$ and $\nu$ and $e^{-}$have the same values of $L$. Let us put $L(\nu)=$ $L\left(e^{-}\right)=1$. By definition, the lepton numbers of antiparticles are opposite to the lepton numbers of particles. We have $L(\bar{\nu})=L\left(e^{+}\right)=-1$. We also assume that the lepton numbers of proton, neutron and other hadrons are

equal to zero. The conservation of the lepton number explains the negative result of the Davis experiment.

\section{Discovery of muon neutrino. The Brookhaven neutrino experiment}

Introduction 
The discovery of the second (muon) neutrino was a great event in physics. It was proved that two different neutrinos $\nu_{e}$ and $\nu_{\mu}$ corresponded to two different (in mass) leptons $e$ and $\mu$. Now we know that with the discovery of $\nu_{\mu}$ it was established that in addition to the first family of leptons $\left(\nu_{e}, e\right)$ there existed the second family $\left(\nu_{\mu}, \mu\right)$.

The Brookhaven neutrino experiment was the first experiment with high energy neutrinos originating from decays of pions, kaons and muons produced at accelerators. As we will see later, important discoveries were made in high energy accelerator neutrino experiments.

When the universal V-A theory of weak interaction was formulated by Feynman and Gell-Mann, Marshak and Sudarshan they considered only one type of neutrinos.

There was, however, an idea of many physicists that neutrinos which take part in the weak interaction together with an electron and a muon could be different 28 Let us call neutrinos which participate in weak processes together with electrons and neutrinos which participate in weak processes together with muons, correspondingly, the electron and muon neutrinos $\left(\nu_{e}\right.$ and $\left.\nu_{\mu}\right)$. The charged current of the current $\times$ current theory takes in this case the form

$$
j^{\alpha}=2\left(\bar{p}_{L} \gamma^{\alpha} n_{L}+\bar{\nu}_{\mu L} \gamma^{\alpha} e_{L}+\bar{\nu}_{e L} \gamma^{\alpha} \mu_{L}\right)
$$

Are $\nu_{e}$ and $\nu_{\mu}$ the same or different particles? The answer to this fundamental question was obtained in the famous Brookhaven neutrino experiment in 1962 37 .

The first indication that $\nu_{e}$ and $\nu_{\mu}$ are different particles was obtained from the data on the search for the decay $\mu \rightarrow e \gamma$. If $\nu_{e}$ and $\nu_{\mu}$ are identical particles, the $\mu \rightarrow e \gamma$ decay is allowed. The probability of the decay $\mu \rightarrow e \gamma$ in the theory with the $W$-boson was calculated in $[38] 29$ soon after the V-A theory has been proposed. It was found that the ratio $R$ of the probability of the decay $\mu^{+} \rightarrow e^{+} \gamma$ to the probability of the decay $\mu^{+} \rightarrow e^{+}+\nu+\bar{\nu}$ was given by

$$
R \simeq \frac{\alpha}{24 \pi} \simeq 10^{-4}
$$

\footnotetext{
${ }^{28}$ Pontecorvo [36] remembered "...f for people working with muons in the old times, the question about different types of neutrinos has always been present. True, later on many theoreticians forgot all about it and some of them "invented" again the two neutrinos....

${ }^{29}$ Such a theory is a non renormalizable one. In 38 , the cut-off $\Lambda \simeq m_{W}$ was applied.
} 
The decay $\mu \rightarrow e \gamma$ was not observed in experiment. At the time of the Brookhaven experiment, for the upper bound of the ratio $R$ much smaller than (77) value

$$
R<10^{-8}
$$

has been found.

A direct proof of the existence of the second (muon) type of the neutrino was obtained by L.M. Lederman, M. Schwartz, J. Steinberger et al. in the first experiment with accelerator neutrinos in 1962. The idea of the experiment was proposed by B.Pontecorvo in 1959 [39].30

A beam of $\pi^{+}$'s in the Brookhaven experiment was obtained by the bombardment of a Be target by protons with an energy of $15 \mathrm{GeV}$. In the decay channel (about $21 \mathrm{~m}$ long) practically all $\pi^{+}$'s decay. After the channel there was shielding material (13.5 m of iron), in which charged particles were absorbed. After the shielding there was neutrino detector (aluminium spark chamber, 10 tons) in which the production of charged leptons was observed.

The dominant decay channel of the $\pi^{+}$-meson is

$$
\pi^{+} \rightarrow \mu^{+}+\nu_{\mu}
$$

According to the universal $V-A$ theory, the ratio $R$ of the width of the decay

$$
\pi^{+} \rightarrow e^{+}+\nu_{e}
$$

to the width of the decay (79) is given by the relation (60). Thus, the decay $\pi^{+} \rightarrow e^{+} \nu_{e}$ is strongly suppressed with respect to the decay $\pi^{+} \rightarrow \mu^{+}+\nu_{\mu}$.

The reason for this suppression can be easily understood. Indeed, let us consider the decay (80) in the rest frame of the pion. The helicity of the neutrino is equal to -1 . If we neglect the mass of the $e^{+}$, the helicity of the positron will be equal to +1 (the helicity of the positron will be in this case the same as the helicity of the antineutrino). Thus, the projection of the total angular momentum on the neutrino momentum will be equal to -1 . The spin of the pion is equal to zero and consequently the process (80) in the limit $m_{e} \rightarrow 0$ is forbidden. These arguments explain the appearance of

\footnotetext{
${ }^{30}$ B. Pontecorvo came to the idea of such an experiment thinking about a possible neutrino program at Meson Factories which were under construction at different places. "At the Laboratory of Nuclear Problems of JINR (Dubna) in 1958 a proton relativistic cyclotron was being designed with a beam energy $800 \mathrm{MeV}$ and beam current $500 \mathrm{~A}$. I started to think about experimental research program for such an accelerator" 36. The Dubna Meson Factory eventually was not built.
} 
the small factor $\left(\frac{m_{e}}{m_{\mu}}\right)^{2}$ in (60). From (60) it follows that the neutrino beam in the Brookhaven experiment was practically a pure $\nu_{\mu}$ beam (with a small admixture of $\nu_{e}$ from decays of muons and kaons).

Neutrinos, emitted in the decay (79), produce $\mu^{-}$in the process

$$
\nu_{\mu}+N \rightarrow \mu^{-}+X
$$

If $\nu_{\mu}$ and $\nu_{e}$ were the same particles, neutrinos from the decay (79) would produce also $e^{-}$in the reaction

$$
\nu_{\mu}+N \rightarrow e^{-}+X
$$

Due to the $\mu-e$ universality of the weak interaction one could expect to observe in the detector practically equal numbers of muons and electrons.

In the Brookhaven experiment 29 muon events were detected. The observed six electron candidates could be explained by the background. The measured cross section was in agreement with the $V-A$ theory. Thus, it was proved that $\nu_{\mu}$ and $\nu_{e}$ were different particles.

In 1963, with the invention of the magnetic horn at the CERN laboratory the intensity and purity of neutrino beams were greatly improved. In a more precise 45 ton spark-chamber experiment and in the large bubble chamber experiment the Brookhaven result was fully confirmed.

The results of the Brookhaven and other experiments suggested that the total electron and muon lepton numbers $L_{e}$ and $L_{\mu}$, which are called flavor lepton numbers, were conserved:

$$
\sum_{i} L_{e}^{(i)}=\text { const; } \quad \sum_{\mathrm{i}} \mathrm{L}_{\mu}^{(\mathrm{i})}=\text { const }
$$

The flavor lepton numbers of particles are given in Table I. The lepton numbers of antiparticles are opposite to the lepton numbers of the corresponding particles.

Table I

Flavor lepton numbers of particles

\begin{tabular}{|cccc|}
\hline Lepton number & $\nu_{e} e^{-}$ & $\nu_{\mu} \mu^{-}$ & hadrons, $\gamma$ \\
\hline$L_{e}$ & 1 & 0 & 0 \\
$L_{\mu}$ & 0 & 1 & 0 \\
\hline
\end{tabular}


We know now that the notion of the flavor lepton number is an approximate one. It is valid only if we neglect small neutrino masses. The conservation laws (83) are violated in neutrino transitions (oscillations) which are due to small neutrino masses and neutrino mixing. Later we will discuss neutrino masses, mixing and oscillations in detail.

In 1988 the Nobel Prize was awarded to L. Lederman, M. Schwartz and J. Steinberger "for the neutrino beam method and the demonstration of the doublet structure of the leptons through the discovery of the muon neutrino".

\subsection{Strange particles. Quarks. Cabibbo current Introduction}

With the idea of quarks, physics of elementary particles and, in particular, physics of the weak interaction and of the neutrino was changed. If the fundamental weak interaction is the interaction of quarks and leptons, the phenomenological rules $|\Delta S|=1$ and $\Delta Q=\Delta S$, which were established for semi-leptonic decays of strange particles, have a natural explanation. The prediction of the charmed quark was motivated by the Cabibbo mixture of quarks, and the Cabibbo-GIM mixture of quarks implied a symmetry between lepton and quark terms in the charged weak current. This symmetry was based on the fact that the number of lepton pairs $\left(\left(\nu_{e}, e^{-}\right)\right.$and $\left.\left(\nu_{\mu}, \mu^{-}\right)\right)$ was equal to the number of quark pairs $((u, d)$ and $(c, s))$. Taking into account that fields of $d$ and $s$ quarks are mixed it was natural to extend the leptonquark symmetry of the charged current and to assume that neutrinos are also mixed. This implies the assumption that neutrinos have small, nonzero masses.

The current $\times$ current Hamiltonian (62) with CC current (176) is the Hamiltonian of such processes in which $e, \nu_{e}, \mu, \nu_{\mu}, p, n, \pi^{ \pm}$and other non strange particles take part. The strange particles were discovered in cosmic rays in the fifties. Their decays were studied in detail in accelerator experiments. From the investigation of the semi-leptonic decays

$$
\begin{gathered}
K^{+} \rightarrow \mu^{+}+\nu_{\mu}, \quad \Lambda \rightarrow n+e^{-}+\bar{\nu}_{e}, \\
\Sigma^{-} \rightarrow n+e^{-}+\bar{\nu}_{e}, \quad \Xi^{-} \rightarrow \Lambda+\mu^{-}+\bar{\nu}_{\mu}
\end{gathered}
$$

and others the following phenomenological rules were formulated. 
I. The strangeness $S$ in the decays of strange particles is changed by one

$$
|\Delta S|=1 .
$$

Here $\Delta S=S_{f}-S_{i}$, where $S_{i}\left(S_{f}\right)$ is the initial (final) total strangeness of the hadrons. As an example, according to this rule, the decay $\Xi^{-} \rightarrow$ $p+\pi^{-}+e^{-}+\bar{\nu}_{e}$, in which $\Delta S=2$, is forbidden. From the data of experiments for the ratio $R$ of the width of the decay $\Xi^{-} \rightarrow n+\pi^{-}+e^{-}+\bar{\nu}_{e}$ to the total decay width of $\Xi^{-}$the following upper bound was obtained: $R<4 \cdot 10^{-4}$. 31

II. The semi-leptonic decays of strange particles obey the rule

$$
\Delta Q=\Delta S .
$$

Here $\Delta Q=Q_{f}-Q_{i}$ where $Q_{i}\left(Q_{f}\right)$ is the initial (final) total electric charge of hadrons (in the unit of the proton charge). According to this rule, the decay $\Sigma^{+} \rightarrow n+e^{+}+\nu_{e}$ is forbidden. From experimental data it follows that $R<5 \cdot 10^{-6}$.

III. The decays of strange particles are suppressed with respect to the decays of non-strange particles.

In 1964, Gell-Mann and Zweig made the assumption that strange and nonstrange hadrons are bound states of $u, d$ and $s$ quarks. The quantum numbers of the quarks are presented in Table II.

\section{Table II.}

Quantum numbers of quarks ( $Q$ is the charge, $S$ is the strangeness, $B$ is the baryon number)

\begin{tabular}{|cccc|}
\hline Quark & $Q$ & $S$ & $B$ \\
\hline$u$ & $2 / 3$ & 0 & $1 / 3$ \\
\hline$d$ & $-1 / 3$ & 0 & $1 / 3$ \\
\hline$s$ & $-1 / 3$ & -1 & $1 / 3$ \\
\hline
\end{tabular}

From the point of view of the theory of quarks $p, n, \Lambda, \Sigma^{+}, \Xi^{-}$and other baryons are bound states of three quarks ( $p=($ uud $), n=($ udd $), \Lambda=$ (uds), $\Sigma^{+}=$(uus), $\Xi^{-}=(d s s)$, etc.) and $\pi^{+}, K^{-}, \bar{K}^{0}$ and other mesons are bound states of a quark and an antiquark $\left(\pi^{+}=(u \bar{d}), K^{-}=(s \bar{u}), \bar{K}^{0}=\right.$ $(d \bar{s})$, etc. $)$.

\footnotetext{
${ }^{31}$ Here and below we present data given in "The Review of Particle Physics" 46.
} 
One of the first argument $\mathrm{s}$ in favor of the quark structure of the hadrons was obtained from the study of the weak decays of strange particles. In expression (76) for the charged current enter the fields of protons and neutrons. If a proton and a neutron are bound states of quarks we can assume that the fundamental weak interaction is the interaction of charged leptons, neutrinos and quarks.

Let us build hadronic charged currents from the quark fields. The current (76) changes the charge by one. If we accept the Feynman-Gell-Mann, Marshak-Sudarshan prescription (the left-handed components of the fermion fields enter into the weak current) there are only two possibilities to build such currents from the fields of $u, d$ and $s$ quarks:

$$
j_{\alpha}^{\Delta S=0}=2 \bar{u}_{L} \gamma_{\alpha} d_{L} \quad \text { and } \quad \mathrm{j}_{\alpha}^{\Delta \mathrm{S}=0}=2 \overline{\mathrm{u}}_{\mathrm{L}} \gamma_{\alpha} \mathrm{S}_{\mathrm{L}}
$$

The first current changes the charge by one and does not change the strangeness $(\Delta Q=1, \Delta S=0)$. The second current changes the charge by one and the strangeness by one $(\Delta Q=1, \Delta S=1)$. The matrix elements of these currents automatically satisfy $|\Delta S|=1$ and $\Delta Q=\Delta S$ rules.

In order to take into account the rule III (suppression of the decays with the change of the strangeness with respect to the decays in which the strangeness is not changed) and describe decays of different strange particles N. Cabibbo 32] introduced a parameter $\theta_{C}$, which later was called the Cabibbo angle, and assumed that the hadronic charged current was a combination of currents $j_{\alpha}^{\Delta S=0}$ and $j_{\alpha}^{\Delta S=1}$ with the coefficients $\cos \theta_{C}$ and $\sin \theta_{C}$, respectively. Assuming a weak universality Cabibbo suggested that in the total hadronic current

$$
j_{\alpha}^{h}=a j_{\alpha}^{\Delta S=0}+b j_{\alpha}^{\Delta S=1}
$$

the real coefficients $a$ and $b$ would satisfy the condition $a^{2}+b^{2}=1$. From this condition it follows that $a=\cos \theta_{C}$ and $b=\sin \theta_{C}$. The Cabibbo paper was written before the quark hypothesis appeared. He assumed that the current which did not change strangeness and the current which changed the strangeness by one are the $1+i 2$ and $4+i 5$ components of the $S U(3)$ octet current. Cabibbo found that with the parameter he introduced it was possible to describe all data on the semi-leptonic decays of mesons and baryons. From analysis of the data he found that $\sin \theta_{C} \simeq 0.2$.

The Cabibbo current had the form

$$
j_{\alpha}^{\text {Cabbibo }}=2\left(\cos \theta_{C} \bar{u}_{L} \gamma_{\alpha} d_{L}+\sin \theta_{C} \bar{u}_{L} \gamma_{\alpha} s_{L}\right)=\bar{u}_{L} \gamma_{\alpha} d_{L}^{\text {mix }},
$$


where

$$
d_{L}^{\operatorname{mix}}(x)=\cos \theta_{C} d_{L}(x)+\sin \theta_{C} s_{L}(x) .
$$

The total charged current took the form

$$
j_{\alpha}=2\left(\bar{\nu}_{e L} \gamma_{\alpha} e_{L}+\bar{\nu}_{\mu L} \gamma_{\alpha} \mu_{L}+\bar{u}_{L} \gamma_{\alpha} d_{L}^{\text {mix }}\right) .
$$

As it is seen from this expression, the lepton and quark terms have the same form and enter into the current with the same coefficients (universality). However, there was asymmetry in the current (88): there are two lepton terms and one quark term. This asymmetry was connected with the fact that four leptons $\left(e, \nu_{e}, \mu, \nu_{\mu}\right)$ and only three quarks $(u, d, s)$ were known at that time.

\subsection{Charmed quark. Quark mixing}

Some years later it was discovered that the charged current (88) creates some problems. Namely, the current (88) generates neutral currents which change the strangeness by one. Such a neutral current induces the decay

$$
K^{+} \rightarrow \pi^{+}+\nu+\bar{\nu}
$$

with a decay rate which is many orders of magnitude larger than the upper bound obtained in experiments.

The solution of the problem was proposed in 1970 by Glashow, Illiopulos and Maiani (GIM) [41]. They assumed that there existed a fourth "charmed" quark $c$ with the charge $2 / 3$ and there was an additional term in the weak charged current

$$
j_{\alpha}^{\mathrm{GIM}}=2\left(-\sin \theta_{C} \bar{c}_{L} \gamma_{\alpha} d_{L}+\cos \theta_{C} \bar{c}_{L} \gamma_{\alpha} s_{L}\right)
$$

into which the field of the new quark $c$ enters. In the theory with this additional current a "dangerous" neutral current which changes the strangeness does not appear.

The total weak charged currents take the form

$j_{\alpha}(x)=2\left(\bar{\nu}_{e L}(x) \gamma_{\alpha} e_{L}(x)+\bar{\nu}_{\mu L}(x) \gamma_{\alpha} \mu_{L}(x)+\bar{u}_{L}(x) \gamma_{\alpha} d_{L}^{\operatorname{mix}}(x)+\bar{c}_{L}(x) \gamma_{\alpha} s_{L}^{\operatorname{mix}}(x)\right)$,

where

$$
\begin{aligned}
& d_{L}^{\operatorname{mix}}(x)=\cos \theta_{C} d_{L}(x)+\sin \theta_{C} s_{L}(x) \\
& s_{L}^{\text {mix }}(x)=-\sin \theta_{C} d_{L}(x)+\cos \theta_{C} s_{L}(x) .
\end{aligned}
$$


Thus, the field of $d$ and $s$ quarks, which have the same charge $(-1 / 3)$ and differ in their masses, enter into the charged current (91) in the form of the orthogonal combinations $d_{L}^{\mathrm{mix}}(x)$ and $s_{L}^{\operatorname{mix}}(x)$ ("mixed form"). The Cabibbo angle $\theta_{C}$ is the mixing angle.

With the additional $c$-quark the numbers of leptons and quarks are equal and there is symmetry between lepton and quark terms in the current (91).

It would be, however, a full lepton-quark symmetry of the charged current if the neutrino masses were different from zero and the fields of neutrinos with definite masses, like the fields of quarks, enter into the $\mathrm{CC}$ in a mixed form

$$
\begin{aligned}
& \nu_{\mu L}(x)=\cos \theta \nu_{1 L}(x)+\sin \theta \nu_{2 L}(x) \\
& \nu_{e L}(x)=-\sin \theta \nu_{1 L}(x)+\cos \theta \nu_{2 L}(x),
\end{aligned}
$$

where $\nu_{1}(x)$ and $\nu_{2}(x)$ are the fields of neutrinos with masses $m_{1}$ and $m_{2}$ and $\theta$ is the neutrino mixing angle (generally different from $\theta_{C}$ ).

We know now that mixing of quarks exists, neutrino masses are different from zero and neutrino mixing (in a more general form; see later) is confirmed by experiment. The lepton-quark symmetry arguments we presented above were early arguments in favor of the neutrino masses and mixing put forward in the seventies (see [42]).

If the $c$-quark, a constituent of hadrons, exists, in this case a new family of "charmed" particles must exist. This prediction was perfectly confirmed by experiment. In 1974 the $J / \Psi$ particles $\left(m_{J / \Psi} \simeq 3096.9 \mathrm{MeV}\right)$, bound states of $(c-\bar{c})$, were discovered. In 1976, $D^{+}=(c \bar{d}), D^{-}=(\bar{c} d)\left(m_{D^{ \pm}} \simeq 1868.6\right.$ $\mathrm{MeV}), D^{0}=(c \bar{u}), \bar{D}^{0}=(\bar{c} u)\left(m_{D^{ \pm}} \simeq 1864.8 \mathrm{MeV}\right)$ were discovered. Later many charmed mesons and baryons were found in experiment. All data obtained from the investigation of weak decays and neutrino reactions were in agreement with the current $\times$ current theory with the current given by (91).

\section{Discovery of the third charged lepton $\tau$. The third family of leptons and quarks}

We do not know why the muon, the particle which has the same interaction as the electron but with a mass 206.8 times larger than the electron mass, exists (the masses of the electron and the muon are $m_{e}=0.51 \mathrm{MeV}$ and 
$\left.m_{\mu}=105.6 \mathrm{MeV}\right) 32$. In such a situation it was natural to ask whether more heavier than $\mu$ (sequential) lepton(s) exist.

The answer to this question was obtained in experiments which were performed in 1975-77 by M. Perl et al. at the $e^{+}-e^{-}$collider at Stanford [43]. In these experiments, the third lepton $\tau^{ \pm}$was discovered 33 The $\tau$-lepton decays into an electron (muon) and two neutrinos, pion(s) and neutrino etc. Its mass $m_{\tau}=1776.8 \mathrm{MeV}$.

Let us combine a charged lepton, a neutrino and quark fields in the following way

1. $\left(\nu_{e}, e^{-}\right)(u, d)$.

2. $\left(\nu_{\mu}, \mu^{-}\right)(c, s)$.

In the first group (family, generation) enter the fields of the lightest leptons and quarks, and in the second family enter fields of heavier leptons and quarks 34 .

The discovery of the $\tau$ could mean that there exists a third family of leptons and quarks. In this case a third type of the neutrino $\nu_{\tau}$, which takes part in weak processes together with $\tau$, and an additional pair of quarks (the top quark $t$ with electric charge $2 / 3$ and bottom quark $b$ with electric charge $-1 / 3)$ must exist.

Let us notice that at the time when the $\tau$-lepton was discovered, the Standard Model of the electroweak interaction which we will discuss later existed. Due to the $S U(2) \times U(1)$ symmetry of this theory the existence of the $\tau$ requires the existence of $\nu_{\tau}, t, b$.

All these expectations were confirmed by experiment. In $1977 \Upsilon$-particles, a bound state of $(b-\bar{b})$, were discovered at the Fermilab $\left(m_{\Upsilon}=9460.3\right.$ $\mathrm{MeV})$. Later $B^{+}=(b \bar{u})\left(m_{B^{+}}=5279.2 \mathrm{MeV}\right) B^{0}=(d \bar{b})\left(m_{B^{0}}=5279.5\right.$ $\mathrm{MeV})$ and other $B$-mesons, $\Lambda_{b}^{0}=(u b d)\left(m_{\Lambda_{b}}=5629.2 \mathrm{MeV}\right)$ and other bottom baryons were detected and studied in many experiments. The mass of the $b$ quark is equal to $m_{b}=4.20_{-0.07}^{+0.17} \mathrm{GeV}$. In 1995, at the Fermilab the $t$ quark was discovered. The $t$-quark is the heaviest known elementary particle

\footnotetext{
${ }^{32}$ The question which was put many years ago by the Nobel Prize winner I.Rabi "Who ordered the $\mu$-meson?" still has no answer. Now we can also ask who ordered $s, c$ and other quarks

${ }^{33}$ In 1995 M. Perl was awarded the Nobel Prize "for the discovery of the tau lepton".

${ }^{34}$ For quark masses we have: $m_{u}=(1.5-3.3) \mathrm{MeV}, m_{d}=(3.5-6.0) \mathrm{MeV}, m_{s}=104_{-34}^{+26}$ $\mathrm{MeV}, m_{c}=1.27_{-0.11}^{+0.07} \mathrm{GeV}$.
} 
$\left(m_{t}=(171.2 \pm 2.1) \mathrm{GeV}\right)$. The third type of neutrino $\nu_{\tau}$, the partner of the $\tau$-lepton, was observed in 2000 in an experiment performed by the DONUT Collaboration at Fermilab 44]. In this experiment the production of $\tau$ in the process $\nu_{\tau}+(A, Z) \rightarrow \tau+\ldots$. was observed. At the energy of the experiment the $\tau$-lepton decays, producing predominantly a single charged particle at an average distance of $2 \mathrm{~mm}$ from the production point. Nuclear emulsion was used to detect the $\tau$ production. A signature of the event in the emulsion was a track with a kink.

In the case of three generations the charged current takes the form

$$
\begin{aligned}
j_{\alpha}^{C C}(x) & =2\left(\bar{\nu}_{e L}(x) \gamma_{\alpha} e_{L}(x)+\bar{\nu}_{\mu L}(x) \gamma_{\alpha} \mu_{L}(x)+\bar{\nu}_{\tau L}(x) \gamma_{\alpha} \tau_{L}(x)\right. \\
& \left.+\bar{u}_{L}(x) \gamma_{\alpha} d_{L}^{\operatorname{mix}}(x)+\bar{c}_{L}(x) \gamma_{\alpha} s_{L}^{\operatorname{mix}}(x)+\bar{t}_{L}(x) \gamma_{\alpha} b_{L}^{\operatorname{mix}}(x)\right)
\end{aligned}
$$

The Cabibbo-GIM mixing of quarks (92) was generalized for the case of three families of quarks by Kobaya shi and Maskawa in 1973 [45]. They assumed that "mixed" fields $d_{L}^{\operatorname{mix}}(x), s_{L}^{\operatorname{mix}}(x), b_{L}^{\operatorname{mix}}(x)$ were connected with the left-handed components of the fields of $d, s$ and $b$ quarks by the unitary transformation:

$d_{L}^{\operatorname{mix}}(x)=\sum_{q=u, s, b} V_{u q} q_{L}(x), s_{L}^{\operatorname{mix}}(x)=\sum_{q=u, s, b} V_{c q} q_{L}(x), b_{L}^{\operatorname{mix}}(x)=\sum_{q=u, s, b} V_{t q} q_{L}(x)$.

The unitary $3 \times 3$ matrix $V$ is called the Cabibbo-Kobayashi-Maskawa (CKM) mixing matrix. The matrix $V$ is characterized by three mixing angles and one phase which is responsible for $C P$ violation.

Kobayashi and Maskawa showed that in the case of two generations of quarks it is impossible to explain $C P$ violation which was observed in decays of neutral $K$ - mesons. This was a main motivation for the assumption of the existence of the third generation of quarks made in 1973 before the $\tau$ lepton, $b$ and $t$ quarks were discovered. In 2008 Kobayashi and Maskawa were awarded the Nobel Prize for "for the discovery of the origin of the broken symmetry which predicts the existence of at least three families of quarks in nature".

On the basis of the lepton-quark symmetry it was natural to assume that the neutrino fields $\nu_{e}, \nu_{\mu}, \nu_{\tau}$ were also mixed (see [42]):

$$
\nu_{l L}(x)=\sum_{i=1}^{3} U_{l i} \nu_{i L}(x) \quad l=e, \mu, \tau
$$


Here $U$ is the unitary $3 \times 3$ neutrino mixing matrix.

In the theory with the intermediate vector boson $W^{ \pm}$the Lagrangian of the CC weak interaction has the form

$$
\mathcal{L}_{\mathcal{I}}^{\mathcal{C C}}(x)=-\frac{g}{2 \sqrt{2}} j_{\alpha}^{C C}(x) W^{\alpha}(x)+\text { h.c. }
$$

where the charged current $j_{\alpha}^{C C}(x)$ is given by expression (94).

\section{Number of families of quarks and leptons}

How many families of quarks and leptons exist in nature? The answer to this fundamental question was obtained in experiments made at SLC (Stanford) and LEP (CERN). In these experiments the width of the decay

$$
Z^{0} \rightarrow \nu_{l}+\bar{\nu}_{l}, \quad l=e, \mu, \tau, \ldots
$$

was determined . The $Z^{0}$-boson has a mass $m_{Z}=91.1876 \pm 0.0021 \mathrm{GeV}$. Different decay modes of the $Z^{0}$-boson $\left(Z^{0} \rightarrow l^{+}+l^{-}(l=e, \mu, \tau), \quad Z^{0} \rightarrow\right.$ hadrons $)$ were investigated in detail at $\left(e^{+}-e^{-}\right)$colliders.

Neglecting small neutrino masses we have

$$
\sum_{l} \Gamma\left(Z^{0} \rightarrow \nu_{l} \bar{\nu}_{l}\right)=n_{\nu_{f}} \Gamma\left(Z^{0} \rightarrow \nu \bar{\nu}\right)
$$

where $n_{\nu_{f}}$ is the number of neutrino-antineutrino pairs and $\Gamma\left(Z^{0} \rightarrow \nu \bar{\nu}\right)$ is the width of the decay of the $Z^{0}$ into a neutrino-antineutrino pair (this width is known from the Standard Model calculations).

From (99) we find the following relation:

$$
n_{\nu_{f}}=\frac{\sum_{l} \Gamma\left(Z^{0} \rightarrow \nu_{l} \bar{\nu}_{l}\right)}{\Gamma\left(Z^{0} \rightarrow l \bar{l}\right)}\left(\frac{\Gamma\left(Z^{0} \rightarrow l \bar{l}\right)}{\Gamma\left(Z^{0} \rightarrow \nu \bar{\nu}\right)}\right)_{S M}
$$

The first ratio is measured in experiments. The second ratio is known from the SM calculations $\left(\left(\frac{\Gamma\left(Z^{0} \rightarrow \nu \bar{\nu}\right)}{\Gamma\left(Z^{0} \rightarrow l \bar{l}\right)}\right)_{S M}=1.991 \pm 0.001\right)$.

From the data of four LEP experiments it was found [46]

$$
n_{\nu_{f}}=2.984 \pm 0.008
$$

Thus, it was established that the number of different types of neutrinos was equal to three (only $\nu_{e}, \nu_{\mu}, \nu_{\tau}$ exist in nature). Each family of leptons and 
quarks has his own neutrino. We conclude that only three families of leptons and quarks exist in nature 35

\section{Unified theory of weak and electromag- netic interactions. The Standard Model}

\section{Introduction}

The unified theory of weak and electromagnetic interactions (Standard Model) is a theory of interaction of neutrinos, charged leptons and quarks with the $W^{ \pm}, Z^{0}$ bosons and $\gamma$-quanta in a wide range of energies. This theory was confirmed by numerous experiments including very precise LEP (CERN) experiments.

The SM is based on the spontaneously broken local gauge $S U(2) \times U(1)$ symmetry and it is built in such a way to include the charged current of the phenomenological $V-A$ theory and the electromagnetic interaction of charged leptons and quarks.

The SM predicts the existence of the $W^{ \pm}$and $Z^{0}$ bosons. This prediction was confirmed by experiment. In 1984, C. Rubbia and S. Van der Meer were awarded the Nobel Prize "for their decisive contributions to the large project, which led to the discovery of the field particles $\mathrm{W}$ and $\mathrm{Z}$, communicators of weak interaction".

Taking into account radiative corrections, for masses and decay widths of the $W^{ \pm}$and $Z^{0}$ bosons from the Standard Model it was obtained [46]

$$
\begin{aligned}
& \left(m_{W}\right)_{S M}=(80.420 \pm 0.031) \mathrm{GeV}, \quad\left(\Gamma_{W}\right)_{S M}=(2.0910 \pm 0.0007) \mathrm{GeV} \\
& \left(m_{Z}\right)_{S M}=(91.1874 \pm 0.0021) \mathrm{GeV}, \quad\left(\Gamma_{Z}\right)_{S M}=(2.4954 \pm 0.0009) \mathrm{GeV} .
\end{aligned}
$$

These values are in agrement with the measured masses and decay widths:

$$
\begin{gathered}
m_{W}=(80.384 \pm 0.014) \mathrm{GeV}, \quad \Gamma_{W}=(2.085 \pm 0.042) \mathrm{GeV} \\
m_{Z}=(91.1876 \pm 0.0021) \mathrm{GeV}, \quad \Gamma_{W}=(2.4952-0.0023) \mathrm{GeV}
\end{gathered}
$$

\footnotetext{
${ }^{35}$ From these data we can not exclude, however, that there exist neutral leptons with masses larger than $\frac{m_{Z}}{2}$ which can not be produced in decays of the $Z^{0}$-bosons. Thus, we can not exclude the existence of new families in which instead of neutrinos such heavy neutral leptons are present.
} 
The Standard Model predicts a new class of weak interactions: neutral currents. Numerous experimental data confirm this prediction. The standard neutral current is diagonal in quark, charged lepton and neutrino fields and is characterized by $\sin ^{2} \theta_{W}$. The values of this parameter determined from different data $\left(e^{+}-e^{-}\right.$, deep inelastic neutrino-nucleon scattering, P-odd asymmetry in deep-inelastic electron-nucleon scattering, etc.) are compatible with each other. From the fit of all data it was found

$$
\sin ^{2} \theta_{W}=0.23108 \pm 0.00005 .
$$

The Standard Model provides a natural framework for quark mixing. However, the SM can not predict the masses of quarks and charged leptons and the CKM mixing angles.

Neutrino masses are not of the Standard Model Higgs origin. For the generation of small neutrino masses and neutrino mixing a new (or additional) mechanism is needed.

In 1979 S. Glashow, S. Weinberg, and A. Salam were awarded the Nobel prize "for their contributions to the theory of the unified weak and electromagnetic interaction between elementary particles, including, inter alia, the prediction of the weak neutral current" 36. In 1999 G. t'Hooft and M Veltman were awarded the Nobel Prize "for elucidating the quantum structure of electroweak interactions in physics".

In 1967-68, S. Weinberg [47] and A. Salam [48] proposed a new theory which unified the weak and electromagnetic interactions into one electroweak interaction. They built such a theory for the electron neutrino and the electron. Later all three families of leptons and quarks were included in the theory. It is called the Standard Model (SM).

The Standard Model predicted a new class of the weak interaction (Neutral currents), the $W^{ \pm}$and $Z^{0}$ vector bosons and the masses of these particles, the existence of the scalar Higgs boson, etc. All predictions of the Standard Model are in perfect agreement with existing experimental data.

\footnotetext{
${ }^{36}$ In his Nobel lecture S. Weinberg remembered how he came to the idea of the electroweak theory. He tried to apply ideas of local gauge invariance and spontaneous symmetry breaking to strong interaction of hadrons. These attempts failed. "At some point at the fall of 1967, while driving to my office at MIT, it occurred to me that I had been applying the right ideas to the wrong problem. It is not the $\rho$-meson that is massless: it is the photon. And its partner is not the $A 1$, but the massive intermediate vector boson...The weak and electromagnetic interactions could then be described in a unified way..."
} 
Recently it was anounced by the CMS [49] and ATLAS [50] collaborations, working at the LHC accelerator at CERN, that they discovered a Higgs-like boson with mass equal to $125 \mathrm{GeV}$. In order to prove that the discovered new particle is the Standard Model Higgs boson it is necessary to determine its spin and parity.(The SM Higgs boson must be scalar particle with spin equal to zero and positive parity). This will be done in futuure experiments.

Neutrinos played an extremely important role in the establishment of the $S M$. In neutrino experiments fundamental parameters of the theory were determined. Neutrinos played also an important role in the establishment of the quark structure of nucleons and its investigation.

The V-A current $\times$ current theory of the weak interaction, which we considered in the previous sections, has been a very successful theory. It allowed one to describe all experimental data, which existed in the sixties. However, the current $\times$ current theory and also the theory with the intermediate $W^{ \pm}$vector boson were unrenormalizable theories. The infinities at higher orders of perturbation theory could not be excluded in these theories by the renormalization of the masses and other physical parameters.

This was the main reason why, in spite of great phenomenological successes, the current $\times$ current theory of the weak interaction and the theory with the intermediate vector boson were not considered as satisfactory. The Standard Model was born in the sixties in an attempt to build a renormalizable theory of weak interaction. The only renormalizable physical theory that was known at that time was quantum electrodynamics. The renormalizable theory of the weak interaction was built in the framework of the unification of the weak and electromagnetic (electroweak) interactions. This theory was proposed by Weinberg [47] and Salam [48]. The same theory with the unification of the weak and electromagnetic interactions but without the mechanism of spontaneous symmetry breaking (see later) was proposed by Glashow in 1961 [51]. Weinberg and Salam suggested that the SM would be a renormalizable theory but they did not prove that. The renormalizability of the SM was proved in 1971 by t'Hooft [52].

We will briefly discuss now the Standard Model of the electroweak interactions 37 The Standard Model is based on

1. Phenomenological V-A theory of the weak interaction.

\footnotetext{
${ }^{37}$ This section requires some knowledge of the Quantum Field Theory (QFT) and a group theory. Readers who are not familiar with QFT and (or) the theory of groups can simply follow the main ideas of the SM, skipping technical details.
} 
2. Local gauge $S U(2) \times U(1)$ invariance of the Lagrangian of fields of massless quarks, leptons and vector bosons 38 .

3. Minimal interaction of fermions and vector bosons.

4. Spontaneous breaking of symmetry and the Higgs mechanism of the generation of masses of quarks and leptons.

5. Unification of the weak and electromagnetic interactions into one electroweak interaction.

The minimal group which ensures the CC interaction of leptons and quarks with $W^{ \pm}$-bosons is the local $S U(2)$ group if we assume that lefthanded components of the fields of quarks and leptons form doublets (the meaning of primes will be clear later)

$$
\psi_{1 L}=\left(\begin{array}{c}
u_{L}^{\prime} \\
d_{L}^{\prime}
\end{array}\right), \psi_{2 L}=\left(\begin{array}{c}
c_{L}^{\prime} \\
s_{L}^{\prime}
\end{array}\right), \psi_{3 L}(x)=\left(\begin{array}{c}
t_{L}^{\prime} \\
b_{L}^{\prime}
\end{array}\right)
$$

and

$$
\psi_{e L}=\left(\begin{array}{c}
\nu_{e L}^{\prime} \\
e_{L}^{\prime}
\end{array}\right), \psi_{\mu L}=\left(\begin{array}{c}
\nu_{\mu L}^{\prime} \\
\mu_{L}^{\prime}
\end{array}\right), \psi_{\tau L}=\left(\begin{array}{c}
\nu_{\tau L}^{\prime} \\
\tau_{L}^{\prime}
\end{array}\right)
$$

We assume also that the right-handed components of the fields of quark and leptons are singlets.

From the local $S U(2)$ invariance it follows that the minimal interaction includes only the left-handed components of quark and lepton fields and has the form

$$
\mathcal{L}_{I}(x)=\left(-\frac{g}{2 \sqrt{2}} j_{\alpha}^{C C}(x) W^{\alpha}(x)+\text { h.c }\right)-g j_{\alpha}^{3}(x) A^{\alpha 3}(x) .
$$

Here

$$
j_{\alpha}^{C C}=2\left(\bar{u}_{L}^{\prime} \gamma_{\alpha} d_{L}^{\prime}+\bar{c}_{L}^{\prime} \gamma_{\alpha} s_{L}^{\prime}+\bar{t}_{L}^{\prime} \gamma_{\alpha} b_{L}^{\prime}\right)+2 \sum_{l=e, \mu, \tau} \bar{\nu}_{l L}^{\prime} \gamma_{\alpha} l_{L}^{\prime}
$$

is the charged current of the quarks and leptons,

$$
j_{\alpha}^{3}=\sum_{a=1,2,3} \bar{\psi}_{a L} \frac{1}{3} \tau_{3} \gamma_{\alpha} \psi_{a L}+\sum_{l=e, \mu, \tau} \bar{\psi}_{l L} \frac{1}{3} \tau_{3} \gamma_{\alpha} \psi_{l L}
$$

\footnotetext{
${ }^{38}$ The $S U(2)$ group is a group of unitary $2 \times 2$ matrices with determinant equal to 1 ; the phase factors $e^{i \Lambda}(x)(\Lambda(x)$ is a function of $x)$ form a $U(1)$ group.
} 
( $\tau_{3}$ is the third Pauli matrix) and $g$ is a constant connected with the $S U(2)$ group. The field $A^{\alpha 3}(x)$ is the field of neutral vector particles.

We would like to unify the weak and electromagnetic interactions on the basis of the local gauge invariance. The first term of (109) is the Lagrangian of the CC weak interaction. However, the second term violates parity and cannot be identified with the Lagrangian of the electromagnetic interaction.

In order to unify the weak interaction (which maximally violates parity) and the electromagnetic interactions (which conserve parity) in one electroweak interaction we must enlarge the symmetry group. The Standard Model is based on the local gauge $S U(2) \times U(1)$ invariance. This is a minimal enlargement of the $S U(2)$ group which generates the charge current weak interaction.

The $U(1)$ group is the group of the hypercharge $Y$ which is determined by the Gell-Mann-Nishijima relation

$$
Q=I_{3}+\frac{1}{2} Y
$$

where $Q$ is the electric charge (in the unit of the proton charge) and $I_{3}$ is the third component of the isotopic spin.

The invariance under the additional $U(1)$ group can be realized if in addition to the vector $W^{\alpha}$ field (field of vector $W^{ \pm}$bosons) and the field of neutral vector particles $A^{\alpha 3}$ the field of neutral vector particles $B^{\alpha}$ exists.

The Lagrangian of the minimal interaction takes the form

$$
\mathcal{L}_{I}(x)=\left(-\frac{g}{2 \sqrt{2}} j_{\alpha}^{C C}(x) W^{\alpha}(x)+\text { h.c }\right)+\mathcal{L}_{I}^{0}(x) .
$$

Here

$$
\mathcal{L}_{I}^{0}(x)=-g j_{\alpha}^{3}(x) A^{\alpha 3}(x)-g^{\prime}\left(j_{\alpha}^{\mathrm{EM}}(x)-j_{\alpha}^{3}(x)\right) B^{\alpha}(x)
$$

is the Lagrangian of interaction of quarks and neutral vector particles,

$$
j_{\alpha}^{\mathrm{EM}}=\left(\frac{2}{3}\right) \sum_{q=u, c, t} \bar{q}^{\prime} \gamma_{\alpha} q^{\prime}+\left(-\frac{1}{3}\right) \sum_{q=d, s, b} \bar{q}^{\prime} \gamma_{\alpha} q^{\prime}+(-1) \sum_{l=e, \mu, \tau} \bar{l}^{\prime} \gamma_{\alpha} l
$$

is the electromagnetic current of the quarks and leptons and $g^{\prime}$ is a constant connected with the $U(1)$ group.

Up to now we considered fields of massless particles. The Standard Model is based on the Higgs mechanism of the generation of masses of the $W^{ \pm}$- and 
$Z^{0}$ bosons, quarks, lepton and other particles. We will assume that in our system enter scalar complex fields of charged and neutral particles $\left(\phi_{+}\right.$and $\left.\phi_{0}\right)$ and that these fields form the $S U(2)$ doublet

$$
\phi=\left(\begin{array}{c}
\phi_{+} \\
\phi_{0}
\end{array}\right) .
$$

The Lagrangian of the Higgs field is chosen in such a way that the energy of the field reaches a minimum when the value of the field is different from zero. This means that the Higgs vacuum is not an empty state. Moreover due to the symmetry there are many (infinite) degenerate vacuum states. If we choose a definite vacuum field, say,

$$
\phi_{0}=\left(\begin{array}{c}
0 \\
\frac{v}{\sqrt{2}}
\end{array}\right)
$$

we will violate the symmetry ( $v$ is a constant). Such a violation is called spontaneous.

Before spontaneous violation of the symmetry we had a massless complex (charged) $W_{\alpha}$ vector field and two massless real (neutral) vector fields $A_{\alpha}^{3}$ and $B_{\alpha}$. After spontaneous violation of the symmetry, the masses of the $W^{ \pm}$and $Z^{0}$ bosons are generated. The field of $Z^{0}$ bosons is the following combination of $A_{\alpha}^{3}$ and $B_{\alpha}$ fields:

$$
Z_{\alpha}=\frac{g}{\sqrt{g^{2}+g^{\prime 2}}} A_{\alpha}^{3}-\frac{g^{\prime}}{\sqrt{g^{2}+g^{\prime 2}}} B_{\alpha} .
$$

For the masses of the $W^{ \pm}$and $Z^{0}$ bosons we have the following relations:

$$
m_{W}^{2}=\frac{1}{4} g^{2} v^{2}, \quad m_{Z}^{2}=\frac{1}{4}\left(g^{2}+g^{\prime 2}\right) v^{2} .
$$

After spontaneous violation of the symmetry the mass of particles, quanta of the field

$$
A_{\alpha}=\frac{g^{\prime}}{\sqrt{g^{2}+g^{\prime 2}}} A_{\alpha}^{3}+\frac{g}{\sqrt{g^{2}+g^{\prime 2}}} B_{\alpha},
$$

which is an orthogonal to $Z_{\alpha}$, remain equal to zero.

Let us introduce the weak (Weinberg) angle $\theta_{W}$ by the relation

$$
\frac{g^{\prime}}{g}=\tan \theta_{W} .
$$


We have

$$
A_{\alpha}=\cos \theta_{W} B_{\alpha}+\sin \theta_{W} A_{\alpha}^{3}, \quad Z_{\alpha}=-\sin \theta_{W} B_{\alpha}+\cos \theta_{W} A_{\alpha}^{3} .
$$

From (114) and (122) we find the following expression for the Lagrangian of interaction of quarks and leptons with neutral vector particles

$$
\mathcal{L}_{I}^{0}=-\frac{g}{2 \cos \theta_{W}} j_{\alpha}^{\mathrm{NC}} Z^{\alpha}-g \sin \theta_{W} j_{\alpha}^{\mathrm{EM}} A^{\alpha},
$$

where

$$
j_{\alpha}^{\mathrm{NC}}=2 j_{\alpha}^{3}-2 \sin ^{2} \theta_{W} j_{\alpha}^{\mathrm{EM}} .
$$

From (123) we can draw the following important conclusions:

1. The second term of (123) is the Lagrangian of the electromagnetic interaction of quarks and charged leptons if the constants $g$ and $\sin \theta_{W}$ satisfy the following (unification) condition:

$$
g \sin \theta_{W}=e,
$$

where e is the proton charge.

2. The unification of the weak and electromagnetic interaction is possible if in addition to the charged vector $W^{ \pm}$-boson there exists a neutral vector $Z^{0}$-boson with a mass larger than the mass of the $W^{ \pm}$-boson (see relation (119)). As a consequence of the unification a new (neutral current) interaction of quarks, charged leptons and neutrinos with the $Z^{0}$-boson appears.

The Fermi constant is given by the relation

$$
\frac{G_{F}}{\sqrt{2}}=\frac{g^{2}}{8 m_{W}^{2}} .
$$

From this relation and (119) it follows that the parameter $v$ (vacuum expectation value), which characterizes the scale of the $S U(2) \times U(1)$ symmetry breaking, is given by

$$
v=\left(\sqrt{2} G_{F}\right)^{-1 / 2} \simeq 246 \mathrm{GeV} .
$$


From the unification condition (125) and relations (119) it follows that the masses of the $W$ and $Z$ bosons are given by the following relations

$$
m_{W}=\left(\frac{\pi \alpha}{\sqrt{2} G_{F}}\right)^{1 / 2} \frac{1}{\sin \theta_{W}}, \quad m_{Z}=\left(\frac{\pi \alpha}{\sqrt{2} G_{F}}\right)^{1 / 2} \frac{1}{\sin \theta_{W} \cos \theta_{W}},
$$

where $\alpha=\frac{e^{2}}{4 \pi}$ is the fine structure constant.

The value of the parameter $\sin \theta_{W}$ can be determined from the study of neutral current (NC) processes. Thus, the Standard Model predicts the masses of the $W^{ \pm}$and $Z^{0}$ bosons. This prediction is in an agreement with experimental data.

We will now briefly discuss a much less predictive part of the SM, the Higgs mechanism of the generation of masses of quarks and leptons. In order to generate the masses of fermions we need to assume that the total Lagrangian of the system contains an $S U(2) \times U(1)$ invariant Lagrangian of a Yukawa interaction of fermions and Higgs boson. For example, the Lagrangian

$$
\mathcal{L}_{Y}^{\text {down }}(x)=-\frac{\sqrt{2}}{v} \sum_{a=1, . . q_{R}^{\prime}=d_{R}^{\prime}, . .} \bar{\psi}_{a L}(x) M_{a ; q}^{\text {down }} q_{R}^{\prime}(x) \phi(x)+\text { h.c. },
$$

after spontaneous violation of the symmetry generates masses of the $d, s$ and $b$ quarks. The matrix $M^{\text {down }}$ in (129) is a complex $3 \times 3$ matrix. The Standard Model does not put any constraints on this matrix. After the diagonalization of the matrix $M^{\text {down }}$ and another similar matrix $M^{\text {up }}$ we find

$$
q_{L}^{\prime}=\sum_{q=d, \ldots} V_{q_{L}^{\prime} q_{L}}^{\text {down }} q_{L}\left(q_{L}^{\prime}=d_{L}^{\prime}, \ldots\right) \quad q_{L}^{\prime}=\sum_{q=u, \ldots} V_{q_{L}^{\prime} q_{L}}^{\text {up }} q_{L} \quad\left(q_{L}^{\prime}=u_{L}^{\prime}, \ldots\right)
$$

Here $V^{\text {down }}$ and $V^{\text {up }}$ are the unitary $3 \times 3$ matrices and $q_{L}$ is the left-handed component of the field of $q$-quark with mass $m_{q}(q=u, c, t, d, s, b)$.

For the lepton fields we have

$$
l_{L}^{\prime}=\sum_{l=e, \mu, \tau} V_{l_{L}^{\prime} l_{L}}^{\mathrm{lep}} l_{L}\left(l_{L}^{\prime}=e_{L}^{\prime}, \mu_{L}^{\prime}, \tau_{L}^{\prime}\right)
$$

where $l_{L}$ is the left-handed component of the field of the lepton $l$ with mass $m_{l}(l=e, \mu, \tau)$. 
Similar relations connect the primed right-handed components of the fields of quarks and leptons and the right-handed components of fields of quarks and leptons with definite masses.

It is important that the matrices $V^{\text {down }}$ and $V^{\text {up }}$ are different and unitary. From (110), (130) and (131) we obtain the following expression for the charged current:

$$
j_{\alpha}^{C C}=2\left(\bar{u}_{L} \gamma_{\alpha} d_{L}^{\mathrm{mix}}+\bar{c}_{L} \gamma_{\alpha} s_{L}^{\mathrm{mix}}+\bar{t}_{L} \gamma_{\alpha} b_{L}^{\mathrm{mix}}\right)+2 \sum_{l=e . \mu, \tau} \bar{\nu}_{l L} \gamma_{\alpha} l_{L}
$$

Here

$$
q_{L}^{\mathrm{mix}}=\sum_{q=d, s, b} V_{q_{L}^{\mathrm{mix}} q_{L}} q_{L}, \quad q_{L}^{\mathrm{mix}}=d_{L}^{\mathrm{mix}}, s_{L}^{\mathrm{mix}}, b_{L}^{\mathrm{mix}}
$$

where

$$
V=V^{\mathrm{L}, \mathrm{up} \dagger} V^{\mathrm{L}, \mathrm{down}}
$$

is the $3 \times 3$ unitary mixing Cabibbo-Kobayashi-Maskawa mixing matrix and

$$
\nu_{l L}=\sum_{l_{1}=e, \mu, \tau}\left(V^{\mathrm{lep}}\right)_{l_{L} l_{1 L}}^{\dagger} \nu_{l_{1} L}^{\prime}
$$

Taking into account the unitarity of the matrices which connect $L(R)$-components of primed fields with $L(R)$-components of fields of particles with definite masses it is easy to show that in the neutral current and in the electromagnetic current we must change primed fields of quarks, leptons and neutrinos by the corresponding physical non primed fields. This means that the NC of the SM does not change strangeness, charm etc.

From discussion of the Higgs mechanism for quarks and charged leptons we could make the following conclusions

1. The Higgs mechanism provides a natural framework for the unitary CKM mixing of quarks in the charged current. It leaves electromagnetic and neutral currents diagonal over fields.

2. However, the Standard Model cannot predict masses of quarks and charged leptons and CKM mixing angles. In the SM these quantities are parameters which have to be determined from experimental data.

What about neutrino masses and mixing in the Standard Model? Many people claim that in the Standard Model neutrinos are massless two-component 
particles. If we assume that there are no right-handed fields $\nu_{l R}^{\prime}$, in this case the corresponding Yukawa interaction can not be built and flavor neutrinos $\nu_{l L}$ will be massless two-component particles. But this is equivalent to assume from the very beginning that neutrinos are the Landau, Lee and Yang and Salam two-component massless particles 39

We can generate neutrino masses by the standard Higgs mechanism in the same way as masses of quarks and charged leptons were generated. In this case neutrino masses would be proportional to the parameter $v$ and we could expect that they are of the same order of magnitude as the masses of other fermions, partners of neutrinos.

Let us consider for illustration the masses of the quarks and leptons of the third family. We have

$$
\begin{aligned}
m_{t} \simeq 1.7 \cdot 10^{2} \mathrm{GeV} & m_{b} \simeq 4.7 \mathrm{GeV} \\
m_{3} \leq 2.210^{-9} \mathrm{GeV} & m_{\tau} \simeq 1.8 \mathrm{GeV}
\end{aligned}
$$

The masses of $t, b$ and $\tau$ differ by not more than 2 orders of magnitude. The neutrino masses differ from the masses of quarks and charged leptons by (at least) (9-11) orders of magnitude. It is very unlikely that the masses of quarks, leptons and neutrinos are of the same Higgs origin. For neutrino masses a new (or additional) mechanism is needed. A possible mechanism of the generation of small neutrino masses will be discussed briefly later.

\section{Neutrino and discovery of Neutral Cur- rents}

\section{Introduction}

The discovery of the neutral currents in the Gargamelle neutrino experiment at CERN in 1973 opened a new era in the physics of the weak and electromagnetic interactions. The Gargamelle result was the first confirmation of the approach based on the idea of the unification of these interactions.

At the beginning of the seventies the Glashow-Weinberg-Salam model was considered as a correct strategy and one of the possible models. However,

\footnotetext{
${ }^{39}$ Originally, the Standard Model was built with massless two-component neutrinos. It was natural in 1967 for the authors of the Standard Model to make this assumption.
} 
after the Gargamelle discovery of NC neutrino processes, detailed investigations of the effects of $\mathrm{NC}$ in deep inelastic electron(muon)-nucleon scattering and in atomic transitions, the discovery of $W^{ \pm}$and $Z^{0}$ bosons and precise measurement of their masses, high precision studies of different electroweak processes at the $e^{+}-e^{-}$colliders SLC(Stanford) and LEP (CERN) fully confirmed the minimal Glashow-Weinberg-Salam model. This model became the Standard Model of the weak and electromagnetic interactions. It perfectly describes the existing electroweak data.

Up to now, however, there is no proof of the correctness of the standard Higgs mechanism. The search for the scalar Higgs boson and the investigation of the mechanism of the symmetry breaking are first priority problems for experiments at the LHC collider at CERN.

Neutral currents were discovered in 1973 at CERN. This was the first confirmation of the unified theory of weak and electromagnetic interactions.

Due to the exchange of the $W$-boson between lepton and quark vertices $\nu_{\mu}\left(\bar{\nu}_{\mu}\right)$ produce $\mu^{-}\left(\mu^{+}\right)$in the inclusive processes

$$
\nu_{\mu}+N \rightarrow \mu^{-}+X, \quad \bar{\nu}_{\mu}+N \rightarrow \mu^{+}+X .
$$

Here $X$ means any possible final state of hadrons. If $Q^{2} \ll m_{W}^{2}\left(Q^{2}\right.$ is the square of the momentum transfer) the effective Hamiltonian of the processes (137) has the form

$$
\mathcal{H}^{C C}=\frac{G_{F}}{\sqrt{2}} 2 \bar{\mu}_{L} \gamma^{\alpha} \nu_{\mu L} j_{\alpha}^{C C}+\text { h.c. }
$$

where $j_{\alpha}^{C C}$ is the quark charged current and $G_{F}$ is the Fermi constant (he Fermi constant has the following numerical value $\left.G_{F}=1.166364(5) \cdot 10^{-5} \mathrm{GeV}^{-2}\right)$. In the seventies the $\mathrm{CC}$ processes (137) were intensively studied in neutrino experiments at the Fermilab and CERN. These experiments were very important for the establishment of the quark structure of the nucleon.

If in addition to the $\mathrm{CC}$ interaction there exists also the $\mathrm{NC}$ interaction, in this case processes

$$
\nu_{\mu}+N \rightarrow \nu_{\mu}+X, \quad \bar{\nu}_{\mu}+N \rightarrow \bar{\nu}_{\mu}+X
$$

induced by the exchange of the $Z$-boson between neutrino and quark vertices become possible. The signature of such processes is hadrons in the final state 
(no muons). The effective SM Hamiltonian of the processes (139) has the form

$$
\mathcal{H}^{N C}=\frac{G_{F}}{\sqrt{2}} 2 \bar{\nu}_{\mu L} \gamma^{\alpha} \nu_{\mu L} j_{\alpha}^{N C}+\text { h.c. },
$$

where $j_{\alpha}^{N C}$ is the neutral current of quarks. Thus, in the framework of the Standard Model CC and NC interactions are characterized by the same Fermi constant. We can expect that the cross section of the processes (137) and (139) are comparable.

The processes (139) were observed in the large bubble chamber Gargamelle at CERN in 1973. The bubble chamber Gargamelle (4.8 m long, $2 \mathrm{~m}$ in diameter, filled with 18 tonnes of liquid Freon) was built specially for the study of neutrino processes. At the first meeting of the collaboration in Milan (1968), where the neutrino program was discussed, the search for NC induced processes had the eighth priority. The main aim of the experiment was an investigation of the structure of a nucleon through the observation of CC processes (137). The Glashow-Weinberg-Salam model was considered at that time only as one of the possibilities.

At the beginning of 1973 one event of the NC process

$$
\nu_{\mu}+e \rightarrow \nu_{\mu}+e
$$

was found in the Gargamelle chamber. Taking into account that the background for (141) is very small (less than 1\%) this one event motivated the intensive search for hadronic NC-induced processes (137) which have cross sections about two orders of magnitude larger than the cross section of the NC leptonic process (141).

The main problem in the search for hadronic NC processes was a background from neutrons produced in $\mathrm{CC}$ neutrino interactions in the surrounding materials. The proof of the neutrino origin of $\mathrm{NC}$ hadronic events followed from the fact that the ratio of selected $\mathrm{NC}$ events and $\mathrm{CC}$ events did not depend on the longitudinal and radial distances, whereas hadronic events of the neutron origin would have shown strong dependence on the distance. Obviously, the large size of the bubble chamber was very important for the detection of $\mathrm{NC}$ events. In the first Gargamelle publication [54] for the ratio $R$ of the number of $\mathrm{NC}$ and $\mathrm{CC}$ events the following values were given

$$
R_{\nu}=0.21 \pm 0.03, \quad R_{\bar{\nu}}=0.45 \pm 0.09 .
$$

In the beginning these data were confirmed by the HPWF collaboration working at the Fermilab. However, later the HPWF collaboration modified 
their detector and for the ratio $R_{\nu}$ they announced a result compatible with zero $\left(R_{\nu}=0.05 \pm 0.05\right)$. For about one year many people at CERN and other places did not believe in the correctness of the Gargamelle result.

By the middle of 1974 the Gargamelle collaboration doubled their statistics and confirmed their original result. The HPWF collaboration made a new measurement and also confirmed the Gargamelle finding. This result was confirmed by other Fermilab neutrino experiments. The discovery of the neutral currents was firmly established.

The eighties and nineties were the years of intensive study of different NC-induced processes. The effects of neutral currents were observed in the experiments on the measurement of the asymmetry in the deep inelastic scattering of polarized electrons (and muons) on an unpolarized nucleon target and on the study of atomic processes 40, in experiments on the study of $\nu_{\mu}\left(\bar{\nu}_{\mu}\right)+e \rightarrow \nu_{\mu}\left(\bar{\nu}_{\mu}\right)+e$ processes, etc. All these data were in perfect agreement with the SM. The values of the parameter $\sin ^{2} \theta_{W}$ obtained from the data of different experiments are in a good agreement with each other. From the measurement of the cross sections of NC neutrino reactions (139) and $\mathrm{CC}$ neutrino reactions (137) it was obtained [55]

$$
\sin ^{2} \theta_{W}=0.2277 \pm 0.0016 .
$$

\section{Neutrino masses, mixing and oscillations}

\subsection{Earliest ideas of neutrino masses and oscillations \\ Introduction}

The earliest ideas of neutrino masses, mixing and oscillations were based on arguments like an analogy between weak interactions of leptons and hadrons (quarks), the Nagoya model with the neutrino as a constituent of the proton and other baryons, etc.

After the great success of the theory of the two-component massless neutrinos, for many years these ideas were not shared by the majority of physicists.

\footnotetext{
${ }^{40} \mathrm{In}$ such experiments, the effect of interference of diagrams with the exchange of $\gamma$ and $Z$ was revealed.
} 
Of course, it was absolutely unknown in the seventies whether neutrinos had small masses and, if they had masses, whether they were mixed. However, understanding of neutrino oscillations as an interference phenomenon made it clear (S. Bilenky and B. Pontecorvo [67]) that

1. Experiments on the search for neutrino oscillations constitute the most sensitive way to look for small neutrino mass-squared differences.

2. Experiments with neutrinos from different facilities are sensitive to different values of neutrino mass-squared differences. Neutrino oscillations must be searched for in all possible neutrino experiments (solar, atmospheric, reactor, accelerator).

This strategy was summarized in [67]. After many years of efforts it brought success.

The first idea of neutrino masses and oscillations was suggested in 195758 by B. Pontecorvo [57, 58]. At that time the Gell-Mann and Pais [56] theory of $K^{0} \rightleftarrows \bar{K}^{0}$ mixing and oscillations was confirmed by experiment. Pontecorvo was fascinated by the idea of particle-mixing and oscillations and thought about a possibility of oscillations in the lepton world. In such a way he came to the idea of neutrino oscillations which was a very courageous idea at the time when there was a common opinion that the neutrino is a two-component massless particle.

Before discussing neutrino oscillations let us briefly consider $\left(K^{0}-\bar{K}^{0}\right)$ mixing and oscillations which were studied in detail in many experiments. $K^{0}$ and $\bar{K}^{0}$ are particles with strangeness equal to +1 and -1 , respectively. They are produced in hadronic processes $\left(\pi_{-}+p \rightarrow K^{0}+\Lambda\right.$ etc $)$ in which the strangeness is conserved. For the states of $K^{0}$ and $\bar{K}^{0}$ we have

$$
H_{0}\left|K^{0}\right\rangle=m\left|K^{0}\right\rangle, \quad H_{0}\left|\bar{K}^{0}\right\rangle=m\left|\bar{K}^{0}\right\rangle .
$$

Here $H_{0}$ is the sum of the free Hamiltonian and Hamiltonians of the strong and electromagnetic interactions, $\left|K^{0}\right\rangle$ and $\left|\bar{K}^{0}\right\rangle$ are states of $K^{0}$ and $\bar{K}^{0}$ (in the rest frame) and $m$ is their mass. The arbitrary phase of $\left|K^{0}\right\rangle$ and $\left|\bar{K}^{0}\right\rangle$ can be chosen in such a way that

$$
\left|\bar{K}^{0}\right\rangle=C P\left|K^{0}\right\rangle,
$$

where $C$ is the operator of the charge conjugation and $P$ is the operator of the space inversion. 
The weak interaction does not conserve strangeness. Eigenstates of the total Hamiltonian, which includes the Hamiltonian of the weak interaction, are superpositions

$$
\left|K_{S}^{0}\right\rangle=p\left|K^{0}\right\rangle+q\left|\bar{K}^{0}\right\rangle, \quad\left|K_{L}^{0}\right\rangle=p\left|K^{0}\right\rangle-q\left|\bar{K}^{0}\right\rangle .
$$

From the normalization condition of the states $\left|K_{S, L}^{0}\right\rangle$ it follows that the coefficients $p$ and $q$ satisfy the condition $|p|^{2}+|q|^{2}=1$. From (146) we find the following relations

$$
\left|K^{0}\right\rangle=\frac{1}{2 p}\left(\left|K_{S}^{0}\right\rangle+\left|\bar{K}_{L}^{0}\right\rangle\right), \quad\left|\bar{K}^{0}\right\rangle=\frac{1}{2 q}\left(\left|K_{S}^{0}\right\rangle-\left|\bar{K}_{L}^{0}\right\rangle\right) .
$$

Thus, the states of particles with definite strangeness $K^{0}$ and $\bar{K}^{0}$ are superpositions ("mixtures") of the states of particles with definite masses and widths $K_{S}^{0}$ and $\bar{K}_{L}^{0}$, eigenstates of the total effective nonhermitian Hamiltonian $H$ :

$$
H\left|K_{S, L}^{0}\right\rangle=\lambda_{S, L}\left|K_{S, L}^{0}\right\rangle .
$$

Here

$$
\lambda_{S, L}=m_{S, L}-\frac{i}{2} \Gamma_{S, L}
$$

where $m_{S, L}$ and $\Gamma_{S, L}$ are the mass and the total width of $K_{S}^{0}\left(K_{L}^{0}\right)$. From experimental data it follows that the lifetimes of $K_{S}^{0}$ (Short-lived) and $K_{L}^{0}$ (Long-lived) are given by [46]

$$
\tau_{S}=\frac{1}{\Gamma_{S}}=(0.8953 \pm 0.0005) \cdot 10^{-10} s, \quad \tau_{L}=\frac{1}{\Gamma_{L}}=(5.116 \pm 0.021) \cdot 10^{-8} s .
$$

States with definite masses and widths are evolved in proper time $t$ as follows:

$$
\left|K_{S}^{0}\right\rangle_{t}=e^{-i \lambda_{S} t}\left|K_{S}^{0}\right\rangle, \quad\left|K_{L}^{0}\right\rangle_{t}=e^{-i \lambda_{L} t}\left|K_{L}^{0}\right\rangle
$$

Let us consider the evolution in time of a state $\left|K^{0}\right\rangle$ which describes $K^{0}$ particles produced in a strong process. We will neglect small effects of $C P$ violation. In this case we have $p=q=\frac{1}{\sqrt{2}}$ and

$$
\left|K_{S}^{0}\right\rangle \simeq\left|K_{1}^{0}\right\rangle=\frac{1}{\sqrt{2}}\left(\left|K^{0}\right\rangle+\left|\bar{K}^{0}\right\rangle\right), \quad\left|K_{L}^{0}\right\rangle \simeq\left|K_{2}^{0}\right\rangle=\frac{1}{\sqrt{2}}\left(\left|K^{0}\right\rangle-\left|\bar{K}^{0}\right\rangle\right) .
$$

Thus, in the case of $C P$-conservation we have the following mixing relations

$$
\left|K^{0}\right\rangle=\frac{1}{\sqrt{2}}\left(\left|K_{1}^{0}\right\rangle+\left|K_{2}^{0}\right\rangle\right), \quad\left|\bar{K}^{0}\right\rangle=\frac{1}{\sqrt{2}}\left(\left|K_{1}^{0}\right\rangle-\left|K_{2}^{0}\right\rangle\right) .
$$


From (151) and (153) we find

$$
\left|K^{0}\right\rangle_{t}=\frac{1}{\sqrt{2}}\left(e^{-i \lambda_{S} t}\left|K_{1}^{0}\right\rangle+e^{-i \lambda_{L} t}\left|K_{2}^{0}\right\rangle\right)=g_{+}(t)\left|K^{0}\right\rangle+g_{-}(t)\left|\bar{K}^{0}\right\rangle,
$$

where

$$
g_{+}(t)=\frac{1}{2}\left(e^{-i \lambda_{S} t}+e^{-i \lambda_{L} t}\right), \quad g_{-}(t)=\frac{1}{2}\left(e^{-i \lambda_{S} t}-e^{-i \lambda_{L} t}\right) .
$$

The state $\left|\bar{K}^{0}\right\rangle_{t}$ depends on time in a similar way

$$
\left|\bar{K}^{0}\right\rangle_{t} \simeq \frac{1}{\sqrt{2}}\left(e^{-i \lambda_{S} t}\left|K_{1}^{0}\right\rangle-e^{-i \lambda_{L} t}\left|K_{2}^{0}\right\rangle\right)=g_{+}(t)\left|\bar{K}^{0}\right\rangle+g_{-}(t)\left|K^{0}\right\rangle .
$$

Thus, because of the mixing (147) at $t>0$ the states $\left|K^{0}\right\rangle_{t}$ and $\left|\bar{K}^{0}\right\rangle_{t}$ are superpositions of $\left|K^{0}\right\rangle$ and $\left|\bar{K}^{0}\right\rangle$. The probability of the transition $K^{0} \rightarrow \bar{K}^{0}$ during the time $t$ is given by the expression

$$
P\left(K^{0} \rightarrow \bar{K}^{0} ; t\right)=\left|g_{(}(t)\right|^{2}=\frac{1}{4}\left(e^{-\Gamma_{S} t}+e^{-\Gamma_{L} t}-2 e^{-\frac{1}{2}\left(\Gamma_{S}+\Gamma_{L}\right) t} \cos \Delta m t\right),
$$

where $\Delta m=m_{L}-m_{S}$. Thus, the oscillating term of the probability is determined by the mass difference of the $K_{L}^{0}$ and $K_{S}^{0}$ mesons. Let us stress that this term originates from the interference of the exponents in (156).

The study of the $t$-dependence of the probability $P\left(K^{0} \rightarrow \bar{K}^{0} ; t\right)$ in the region $\Delta m t \geq 1$ allows one to determine the mass difference $\Delta m$. From the analysis of the experimental data it was found 41

$$
\Delta m=(3.483 \pm 0.006) \cdot 10^{-6} \mathrm{eV} .
$$

The measurement of such a small quantity became possible because of the interference nature of the $K^{0} \rightarrow \bar{K}^{0}$ oscillations.

We will discuss now Pontecorvo's idea of neutrino oscillations. Pontecorvo believed in the existence of symmetry between weak interaction of leptons and hadrons and he came first to the idea of muonium -antimuonum oscillations [57] which in the framework of the lepton-hadron symmetry are analogous to $K^{0} \rightleftarrows \bar{K}^{0}$ oscillations. (muonium is the bound state $\left(\mu^{+}-e^{-}\right)$and antimuonum is the bound state of $\left.\left(\mu^{-}-e^{+}\right)\right)$. In [57], Pontecorvo also mentioned neutrino oscillations. This was soon after the two-component theory

\footnotetext{
${ }^{41}$ This value is many orders of magnitude smaller than the masses of the neutral kaons $\left(m_{K^{0}}=497.614 \pm 0.022 \mathrm{MeV}\right)$
} 
of a massless neutrino was proposed and the neutrino helicity was measured in the Goldhaber et al. experiment. Only one type of neutrino was known at that time. Everybody believed that there were only two neutrino states: $\nu_{L}$ and $\bar{\nu}_{R}$. Pontecorvo assumed that

1. Neutrinos had small masses.

2. Lepton number was not conserved.

3. Additional neutrino states $\bar{\nu}_{L}$ and $\nu_{R}$ existed so that $\nu_{L}$ could be transferred into $\bar{\nu}_{L}$ and $\bar{\nu}_{R}$ could be transferred into $\nu_{R}$.

Pontecorvo wrote in [57]: "If the theory of two-component neutrino theory was not valid (which is hardly probable at present) and if the conservation law for neutrino charge took not place, neutrino $\rightarrow$ antineutrino transitions in vacuum would be in principle possible."

A special paper on neutrino oscillations [58] was published by B. Pontecorvo in 1958. At that time R. Davis was doing an experiment with reactor antineutrinos [35] with the aim to test the conservation of the lepton number $L$. Davis searched for the production of ${ }^{37} \mathrm{Ar}$ in the process

$$
\bar{\nu}+{ }^{37} \mathrm{Cl} \rightarrow \mathrm{e}^{-}+{ }^{37} \mathrm{Ar},
$$

which is evidently forbidden if $L$ is conserved. A rumor reached B. Pontecorvo that Davis had seen some events (159). B.Pontecorvo who had earlier been thinking about neutrino oscillations was very excited with a possibility to explain Davis "events" by $\bar{\nu}_{R} \rightarrow \nu_{R}$ oscillations.

He wrote: "Recently the question was discussed [57] whether there exist other mixed neutral particles beside the $K^{0}$ mesons, i.e., particles that differ from the corresponding antiparticles, with the transitions between particle and antiparticle states not being strictly forbidden. It was noted that the neutrino might be such a mixed particle, and consequently there existed a possibility of real neutrino $\rightleftarrows$ antineutrino transitions in vacuum, provided that lepton (neutrino) charge was not conserved. This means that the neutrino and antineutrino are mixed particles, i.e., a symmetric and antisymmetric combination of two truly neutral Majorana particles $\nu_{1}$ and $\nu_{2}$ of different combined parity".

So basically by analogy with the $K^{0}-\bar{K}^{0}$ mixing (153) Pontecorvo assumed that

$$
\left|\bar{\nu}_{R}\right\rangle=\frac{1}{\sqrt{2}}\left(\left|\nu_{1}\right\rangle+\left|\nu_{2}\right\rangle\right), \quad\left|\nu_{R}\right\rangle=\frac{1}{\sqrt{2}}\left(\left|\nu_{1}\right\rangle-\left|\nu_{2}\right\rangle\right)
$$


where $\left|\nu_{1,2}\right\rangle$ are states of Majorana neutrinos $\nu_{1,2}$ with masses $m_{1,2}, 42$

In contrast to $K_{S, L}^{0}$ the neutrinos $\nu_{1,2}$ are stable particles 43 . From (160) we find (in the lab. system)

$$
\left|\bar{\nu}_{R}\right\rangle_{t}=\frac{1}{\sqrt{2}}\left(e^{-i E_{1} t}\left|\nu_{1}\right\rangle+e^{-i E_{2} t}\left|\nu_{2}\right\rangle\right)=\frac{1}{2}\left(g_{+}(t)\left|\bar{\nu}_{R}\right\rangle+g_{-}(t)\left|\nu_{R}\right\rangle\right) .
$$

Here

$$
g_{ \pm}(t)=\left(e^{-i E_{1} t} \pm e^{-i E_{2} t}\right), \quad E_{i}=\sqrt{p^{2}+m_{i}^{2}} \simeq p+\frac{m_{i}^{2}}{2 E}
$$

where $p$ is the neutrino momentum. In neutrino experiments we have $p \gg m_{i}$ and $p \simeq E$ ( $E$ is the neutrino energy).

From (161) and (162) for the transition probabilities we obtain the following expressions:

$$
P\left(\bar{\nu}_{R} \rightarrow \nu_{R}\right)=\frac{1}{2}\left(1-\cos \frac{\Delta m^{2} L}{2 E}\right), \quad P\left(\bar{\nu}_{R} \rightarrow \bar{\nu}_{R}\right)=1-\frac{1}{2}\left(1-\cos \frac{\Delta m^{2} L}{2 E}\right),
$$

where $\Delta m^{2}=m_{2}^{2}-m_{1}^{2}$ and $L \simeq t$ is the distance between neutrino source and neutrino detector.

Thus, in the case of the neutrino oscillations the probability for a reactor antineutrino to survive $P\left(\bar{\nu}_{R} \rightarrow \bar{\nu}_{R}\right)$ depends on the distance $L$. B. Pontecorvo wrote in [58]: “...the cross section of the production of neutrons and positrons in the process of the absorption of antineutrinos from a reactor by protons would be smaller than the expected cross section. It would be extremely interesting to perform the Reines-Cowan experiment at different distances from reactor".

Pontecorvo obviously did not know the value of the neutrino mass-squared difference $\Delta m^{2}$. If it is relatively large, the cosine terms in (163) disappear due to averaging over neutrino energies and distance. In this case $P\left(\bar{\nu}_{R} \rightarrow \bar{\nu}_{R}\right)=P\left(\bar{\nu}_{R} \rightarrow \nu_{R}\right)=\frac{1}{2}$. Discussing this case he wrote :"...a beam of neutral leptons consisting mainly of antineutrinos when emitted from a nuclear reactor, will consist at some distance $L$ from the reactor of half neutrinos and half antineutrinos."

If $\Delta m^{2}$ is very small, in this case the cosine terms are practically equal to one and the effect of oscillations of reactor antineutrinos could not be

\footnotetext{
${ }^{42}$ If the lepton number $L$ is violated, there is no way to distinguish a neutrino and an antineutrino: they are the same particles. A theory of such particles was proposed by E. Majorana in 1937 24.

${ }^{43}$ No indications in favor of neutrino decays were found.
} 
observed. Pontecorvo noticed in [58]: "...effect of transformation of neutrino into antineutrino and vice versa may be unobservable in laboratory, but will certainly occur, at least, on an astronomic scale".

Let us stress again that the proposal of neutrino oscillations immediately after the great success of the two-component neutrino theory and in the situation when only one type of neutrino was known was a very nontrivial one. The Pontecorvo paper was written at the time when the Davis reactor experiment was not yet finished and candidate-events (159) existed. In order to explain them he had to assume that $\nu_{R}$ interacts with matter. He wrote:" ... it is impossible to conclude a priori that the antineutrino beam which at first is essentially incapable of inducing the reaction in question transforms itself into a beam in which a definite fraction of particles can induce such reaction".

In spite of the fact that the candidate-events (159) disappeared and only an upper bound for the cross section of the process (159) was found in the Davis experiment, Pontecorvo continued to believe in neutrino oscillations. He liked the idea that neutrinos (antineutrinos) produced in weak processes can oscillate into antineutrinos (neutrinos) which have no (standard) weak interaction. He proposed to name such noninteracting neutrinos sterile. The idea of sterile neutrinos is very popular nowadays.

The program of the study of oscillations of reactor antineutrinos, which was outlined by B. Pontecorvo in the very first paper on neutrino oscillations, was realized in the KamLAND experiment about 40 years later. We will discuss this experiment in the next subsection.

After the first paper on the neutrino oscillations Pontecorvo continued to think about this fascinating phenomenon. His belief in neutrino masses was based on the fact that there was no principle (like gauge invariance for photon) which requires the neutrino to be a massless particle.

In the sixties, B. Pontecorvo discussed the problem of neutrino masses with L. Landau, one of the authors of the two-component neutrino theory. Landau agreed with Pontecorvo that after the V-A theory, which was based on the assumption that the left-handed components of all fields entered into weak interaction Hamiltonian, neutrino was not longer special particles and it would be natural for neutrinos to have small masses.

After the discovery of the second neutrino $\nu_{\mu}$ Pontecorvo applied his idea of neutrino oscillations to the case of two types of neutrinos $\nu_{e}$ and $\nu_{\mu}$. In the second paper on neutrino oscillations published in 1967 [59] Pontecorvo considered $\nu_{e} \rightleftarrows \nu_{\mu}, \nu_{e} \rightleftarrows \bar{\nu}_{e L}$ (sterile), $\nu_{e} \rightleftarrows \bar{\nu}_{\mu L}$ (sterile), etc. oscillations and applied the idea of neutrino oscillations to solar neutrinos. 
At that time R. Davis started his famous experiment on the detection of the solar neutrinos in which the radiochemical method of neutrino detection, proposed by B.Pontecorvo in 1946, was used. Solar neutrinos were detected in this experiment via the the observation of the reaction

$$
\nu_{e}+{ }^{37} \mathrm{Cl} \rightarrow \mathrm{e}^{-}+{ }^{37} \mathrm{Ar} .
$$

In the paper [59] B. Pontecorvo wrote: "From an observational point of view the ideal object is the sun. If the oscillation length is smaller than the radius of the sun region effectively producing neutrinos, (let us say one tenth of the sun radius $R_{\odot}$ or 0.1 million $\mathrm{km}$ for ${ }^{8} B$ neutrinos, which will give the main contribution in the experiments being planned now), direct oscillations will be smeared out and unobservable. The only effect on the earth's surface would be that the flux of observable sun neutrinos must be two times smaller than the total (active and sterile) neutrino flux."

The first Davis results was obtained at the end of the sixties (see [60]). It was found that the upper bound of the observed flux of the solar $\nu_{e}$ 's was (2-3) times smaller than the predicted flux. This result created "the solar neutrino problem". In [59] Pontecorvo envisaged the solar neutrino problem. He understood, however, that the prediction of the flux of highenergy ${ }^{8} B$ neutrinos, which gave the major contribution to the event rate in the Davis experiment, was an extremely difficult problem: "Unfortunately, the relative weight of different thermonuclear reactions in the sun and its central temperature are not known well enough to permit a comparison of the expected and observed solar neutrino intensities." It took many years of research to prove that the observed depletion of fluxes of solar neutrinos are effects of neutrino transitions due to neutrino masses, mixing and interaction of neutrinos with matter which we will briefly discuss later.

The first phenomenological scheme of neutrino mixing was proposed by V. Gribov and B. Pontecorvo in 1969 [61]. They assumed that only the left-handed flavor fields $\nu_{e L}(x)$ and $\nu_{\mu L}(x)$ entered into the total Lagrangian. There was a widespread opinion at that time that in this case neutrino masses must be equal to zero. V. Gribov and B. Pontecorvo showed that this is not the case if the total lepton number $L$ is violated. In this case

$$
\begin{aligned}
& \nu_{e L}(x)=\cos \theta \nu_{1 L}(x)+\sin \theta \nu_{2 L}(x) \\
& \nu_{\mu L}(x)=-\sin \theta \nu_{1 L}(x)+\cos \theta \nu_{2 L}(x),
\end{aligned}
$$

where $\nu_{1}(x)$ and $\nu_{2}(x)$ are the fields of Majorana neutrinos with masses $m_{1}$ and $m_{2}$ and $\theta$ is the mixing angle. 
The scheme of two-neutrino mixing, proposed by V. Gribov and B. Pontecorvo, was the minimal one. In this scheme:

- The only possible oscillations are $\nu_{e} \rightleftarrows \nu_{\mu}$.

- There are no sterile neutrinos.

- To four states of flavor neutrinos and antineutrinos (left-handed $\nu_{e}, \nu_{\mu}$ and right-handed $\bar{\nu}_{e}, \bar{\nu}_{\mu}$ ) there correspond four states of two massive Majorana neutrinos with helicities \pm 1 .

In [61] the following general expression for the two-neutrino survival probability in vacuum was obtained 44

$$
P\left(\nu_{e} \rightarrow \nu_{e}\right)=1-\frac{1}{2} \sin ^{2} 2 \theta\left(1-\cos \frac{\Delta m^{2} L}{2 E}\right) .
$$

In [61] and later in [62] the effect of vacuum $\nu_{e} \rightleftarrows \nu_{\mu}$ oscillations on the flux of solar $\nu_{e}$ 's on the earth was discussed.

In the eighties the Cabibbo-GIM mixing (92) of $d$ and $s$ quarks was fully established. In the papers [63, 64, 65] neutrino mixing

$$
\begin{aligned}
& \nu_{e L}(x)=\cos \theta \nu_{1 L}(x)+\sin \theta \nu_{2 L}(x) \\
& \nu_{\mu L}(x)=-\sin \theta \nu_{1 L}(x)+\cos \theta \nu_{2 L}(x)
\end{aligned}
$$

was introduced on the basis of the lepton-quark analogy. The main ideas were the following:

1. Neutrinos like all other fundamental fermions (charged leptons and quarks) are massive particles.

2. The mixing is a general feature of gauge theories with a mass generation mechanism based on the spontaneous violation of symmetry. Thus, it is quite natural to assume that fields of neutrinos like fields of quarks enter into the charged current in a mixed form.

In (167), $\nu_{1}(x)$ and $\nu_{2}(x)$ are the fields of neutrinos with masses $m_{1}$ and $m_{2}$. However, in contrast to the Gribov-Pontecorvo scheme, in this scheme the total lepton number is conserved and $\nu_{1,2}$ are the Dirac particles (like

\footnotetext{
${ }^{44}$ Expression (163) corresponds to the case of maximal mixing $\theta=\frac{\pi}{4}$.
} 
quarks). In [63, 64, 65] possible neutrino oscillations in reactor and accelerator neutrino experiments were discussed.

As we have seen earlier, the initial ideas of neutrino masses, mixing and oscillations were based on symmetry (analogy) of weak interactions of leptons and hadrons (and later leptons and quarks). In 1962 Maki, Nakagawa and Sakata [66] introduced the neutrino mixing in the framework of the Nagoya model in which the proton and other baryons were considered as bound states of neutrinos and a vector boson $B^{+}$, "a new sort of matter". At that time the Brookhaven experiment, in which it was proved that $\nu_{e}$ and $\nu_{\mu}$ were different particles, was not yet finished. However, there was an indication, based on the fact the decay $\mu^{+} \rightarrow e^{+}+\gamma$ was not observed, that $\nu_{e}$ and $\nu_{\mu}$ were different types of neutrinos determined by the weak charged current

$$
j_{\alpha}^{C C}=2\left(\bar{\nu}_{e L} \gamma_{\alpha} e_{L}+\bar{\nu}_{\mu L} \gamma_{\alpha} \mu_{L}\right) .
$$

The authors wrote: "We assume that there exists a representation which defines the true neutrinos $\nu_{1}$ and $\nu_{2}$ through orthogonal transformation"

$$
\begin{aligned}
& \nu_{1}=\cos \delta \nu_{e}-\sin \delta \nu_{\mu} \\
& \nu_{2}=\sin \delta \nu_{e}+\cos \delta \nu_{\mu},
\end{aligned}
$$

where $\delta$ is the Cabibbo angle. In [66] it was assumed that the "true neutrino" $\nu_{1}$ was a constituent of baryons and possessed some mass $m_{1}$. Further, the authors assumed that there existed an additional interaction of $\nu_{2}$ with a field of heavy particles $X$ which ensured the difference of masses of $\nu_{2}$ and $\nu_{1}$.

In contrast to [58, 59, 61], in [66] the quantum phenomenon of neutrino oscillations, based on the difference of phases which were gained in propagation of neutrinos with definite masses, was not considered. Nevertheless $\nu_{e} \rightarrow \nu_{\mu}$ transitions were discussed in [66]. The authors wrote: "Weak neutrinos

$$
\begin{aligned}
& \nu_{e}=\cos \delta \nu_{1}+\sin \delta \nu_{2} \\
& \nu_{\mu}=-\sin \delta \nu_{1}+\cos \delta \nu_{2}
\end{aligned}
$$

are not stable due to the occurrence of virtual transition $\nu_{e} \rightleftarrows \nu_{\mu}$ caused by this additional interaction with $\nu_{2}$ ". Moreover, in connection with the Brookhaven neutrino experiment they noticed : ..." a chain of reactions

$$
\begin{aligned}
\pi^{+} & \rightarrow \mu^{+}+\nu_{\mu} \\
\nu_{\mu}+Z & \rightarrow\left(\mu^{-} \text {and } / \text { or }\right) e^{-}
\end{aligned}
$$


is useful to check the two-neutrino hypothesis only when

$$
\left|m_{\nu_{2}}-m_{\nu_{1}}\right| \leq \mathrm{eV}
$$

under the conventional geometry of the experiments. Conversely, the absence of $e^{-}$will be able not only to verify the two-neutrino hypothesis but also to provide an upper limit of the mass of the second neutrino $\nu_{2}$ if the present scheme should be accepted."

The papers $61,63,64,65,66$ were written at the time when only two types of flavor neutrinos $\nu_{e}$ and $\nu_{\mu}$ were known. In [61] it was assumed that there was no conserved lepton number and neutrinos with definite masses $\nu_{1}$ and $\nu_{2}$ were truly neutral Majorana particles. In [63, 64, 65, 66] it was assumed that the total lepton number $L$ was conserved and $\nu_{1}$ and $\nu_{2}$ were Dirac particles $\left(L\left(\nu_{i}\right)=1, L\left(\bar{\nu}_{i}\right)=-1\right)$. After the discovery of the $\tau$-lepton it was natural to assume that there existed (at least) three different types of neutrinos. The mixing relations (165) and (167) were generalized for an arbitrary number $n$ of flavor neutrinos in the following way (see [67]):

$$
\nu_{l L}=\sum_{i=1}^{n} U_{l i} \nu_{i L}, \quad l=e, \mu, \ldots
$$

Here $U$ is a unitary $n \times n$ matrix $\left(U^{\dagger} U=1\right)$. The matrix $U$ is called the mixing matrix. As we will see later, the existing neutrino oscillation data can be described if we assume that $U$ is the $3 \times 3$ matrix. This matrix is usually called the Pontecorvo-Maki-Nakagawa-Sakata (PMNS) mixing matrix in order to pay tribute to the pioneering contribution of these authors to the neutrino mixing and oscillations.

The mixing (173) is not the most general one. In the most general case we have (see [68])

$$
\begin{aligned}
& \nu_{l L}=\sum_{i=1}^{n+n_{s t}} U_{l i} \nu_{i L} \\
& \nu_{s L}=\sum_{i=1}^{n+n_{s t}} U_{s i} \nu_{i L},
\end{aligned}
$$

where the index $s$ takes $n_{s t}$ values and $U$ is a unitary $\left(n+n_{s t}\right) \times\left(n+n_{s t}\right)$ mixing matrix. The fields $\nu_{s L}$ are fields of the sterile neutrinos which have no standard weak interaction. Due to the mixing (174) transitions between 
flavor neutrinos $\nu_{l} \rightleftarrows \nu_{l^{\prime}}$ as well as transitions between flavor and sterile neutrinos $\nu_{l} \rightleftarrows \nu_{s L}$ are possible.

In spite of the fact that in the seventies some plausible arguments for small nonzero masses were given and a general phenomenological theory of neutrino mixing and oscillations was developed there was no so much interest in neutrino masses and oscillations at that time: the idea of massless twocomponent neutrinos was still the dominant one. In the first review on neutrino oscillations [67] only about ten neutrino oscillation papers existing at that time were referred to.

\subsection{Neutrino oscillations at the time when neutrino masses started to be considered as a signature of the physics beyond the SM}

\section{Introduction}

In the eighties and nineties with new solar neutrino experiments and the increase in the number of detected atmospheric neutrino events the evidence in favor of neutrino masses and oscillations, coming from these experiments, became stronger and stronger. However, the interpretation of the data of solar neutrino experiments depended on the Standard Solar Model. In experiments with neutrinos of terrestrial origin (reactor and accelerator neutrinos) no positive indications in favor of neutrino oscillations were found. In 1998, the situation with neutrino oscillations drastically changed.

The situation with neutrino masses and the mixing problem drastically changed at the end of the seventies with appearance of the models of grand unification (GUT). In these models leptons and quarks enter into the same multiplets, and the generation of masses of quarks and charged leptons in some models naturally lead to nonzero neutrino masses. At that time the famous seesaw mechanism of the neutrino mass generation [71], which could explain the smallness of the neutrino masses with respect to the masses of quarks and charged leptons, was proposed.

After the appearance of the GUT models and the seesaw mechanism of neutrino mass generation, masses and mixing of neutrinos started to be considered as a signature of the physics beyond the Standard Model. The problem of neutrino masses and oscillations started to attract more and more attention 
of theoreticians and experimentalists. Several short-baseline 45 experiments on the search for neutrino oscillations with reactor and accelerator neutrinos were performed in the eighties. No positive indications in favor of oscillations in these experiments with artificially produced neutrinos were found at that time. 46

On the other hand, indications in favor of oscillations of solar neutrinos were strengthened in the eighties. The second solar neutrino experiment Kamiokande was performed [69]. In this experiment high energy solar neutrinos from the decay ${ }^{8} \mathrm{~B} \rightarrow{ }^{8} \mathrm{Be}+e^{+}+\nu_{e}$ were detected via the observation of the recoil electrons from the elastic $\nu+e \rightarrow \nu+e$ scattering. The ratio of the observed flux of the solar neutrinos to the predicted flux obtained in the Kamiokande experiment was about 1/2.

In the Kamiokande and IMB water Cherenkov detectors high energy muons and electrons, produced by atmospheric muon and electron neutrinos were detected 47 It was found in these experiments that the ratio of the numbers of the $\nu_{\mu}$ and $\nu_{e}$ events was significantly smaller than the (practically model independent) predicted ratio [70]. This effect was called the atmospheric neutrino anomaly. The anomaly could be explained by the disappearance of $\nu_{\mu}$ due to transitions of $\nu_{\mu}$ into other neutrino states.

At the beginning of the nineties two new solar neutrino experiments GALLEX 72] and SAGE [73] were performed. In these experiments, like in the first Davis experiment, Pontecorvo's radiochemical method of neutrino detection was used. Solar $\nu_{e}$ 's were detected via the observation of radioactive ${ }^{71} \mathrm{Ge}$ atoms produced in the process

$$
\nu_{e}+{ }^{71} \mathrm{Ga} \rightarrow \mathrm{e}^{-}+{ }^{71} \mathrm{Ge} .
$$

There are three main sources of $\nu_{e}$ 's in the sun

$$
\text { 1. The } p p \text { reaction } p+p \rightarrow d+e^{+}+\nu_{e}(E \leq 0.42 \mathrm{MeV}) \text {. }
$$

\footnotetext{
${ }^{45}$ Distances between sources and detectors in these experiments were a few hundred meters or less.

${ }^{46}$ Recently, fluxes of $\bar{\nu}_{e}$ 's from reactors were recalculated. It occurred that the fluxes are (3-4) \% higher than the fluxes used in the analysis of old reactor neutrino oscillation data. Thus, these data nowadays are interpreted as an indication in favor of short-baseline neutrino oscillations. New reactor and accelerator neutrino experiments are under preparation in order to check the hypothesis of short-baseline oscillations.

${ }^{47}$ Atmospheric neutrinos are produced mainly in decays of pions produced in processes of interactions of cosmic rays in the atmosphere, and muons which are produced in decays of pions $\left(\pi^{ \pm} \rightarrow \mu^{ \pm}+\nu_{\mu}\left(\bar{\nu}_{\mu}\right), \mu^{ \pm} \rightarrow e^{ \pm}+\nu_{e}\left(\bar{\nu}_{e}\right)+\bar{\nu}_{\mu}\left(\nu_{\mu}\right)\right)$.
} 
2. The ${ }^{7} \mathrm{Be}$ capture $e^{-}+{ }^{7} \mathrm{Be} \rightarrow{ }^{7} \mathrm{Li}+\nu_{e}(E=0.86 \mathrm{MeV})$.

3. The ${ }^{8} \mathrm{~B}$ decay ${ }^{8} \mathrm{~B} \rightarrow{ }^{8} \mathrm{Be}+e^{+}+\nu_{e}(E \leq 15 \mathrm{MeV})$.

The threshold of the $\mathrm{Cl}-\mathrm{Ar}$ reaction (70) is equal to $0.81 \mathrm{MeV}$. Thus, in the Davis experiment mainly ${ }^{8} \mathrm{~B}$ neutrinos can be detected. The threshold of the reaction (175) is equal to $0.23 \mathrm{MeV}$. This means that in the GALLEX and SAGE experiments neutrinos from all reactions of thermonuclear cycles in the sun including low-energy neutrinos from the $p p \rightarrow d e^{+} \nu_{e}$ reaction were detected. This reaction gives the largest contribution to the flux of the solar neutrinos. The flux of the $p p$ neutrinos can be connected with the luminosity of the sun and can be predicted in a model independent way.

The event rates measured in the GALLEX and SAGE experiments were approximately two times smaller than the predicted rates. Thus, in these experiments additional important evidence was obtained in favor of the disappearance of solar $\nu_{e}$ on the way from the central region of the sun, where solar neutrinos are produced, to the earth.

Solar $\nu_{e}$ 's are produced in the central region of the sun and on the way to the earth pass about $7 \cdot 10^{5} \mathrm{~km}$ of the solar matter. It was discovered in the nineties [74] that for neutrino propagation in matter not only masses and mixing but also coherent interaction are important. This interaction gives an additional contribution to the Hamiltonian of a neutrino in matter which is determined by the electron number-density. If the electron density depends on the distance (as in the case of the sun) the transition probabilities between different flavor neutrinos in matter can have the resonance character (MSW effect).

\subsection{Golden years of neutrino oscillations (1998-2004)}

In 1998, in the Super-Kamiokande atmospheric neutrino experiment[75] (Japan) significant up-down asymmetry of the high-energy muon events was observed. Neutrinos produced in the earth atmosphere and coming from above pass distances from about $20 \mathrm{~km}$ to $500 \mathrm{~km}$. Neutrinos coming to the detector from below pass the earth and travel distances from $500 \mathrm{~km}$ to about $12000 \mathrm{~km}$. It was discovered in the Super-Kamiokande experiment that the number of up-going high-energy muon neutrinos was about two times smaller than the number of the down-going high-energy muon neutrinos. Thus, it was proved that the number of observed muon neutrinos depends on the distance which neutrinos passed from a production point in the atmosphere to the detector. 
The Super-Kamiokande atmospheric neutrino result was the first model independent evidence of neutrino oscillations. This result marked a new era in the investigation of neutrino oscillations - an era of experiments with neutrinos from different sources which provide model independent evidence of neutrino oscillations.

In 2002 in the SNO solar neutrino experiment [76] (Canada) model independent evidence of the disappearance of solar $\nu_{e}$ was obtained. In this experiment high-energy solar neutrinos from ${ }^{8} B$-decay were detected through the observation of $\mathrm{CC}$ and $\mathrm{NC}$ reactions. The detection of solar neutrinos through the observation of the $\mathrm{CC}$ reaction allows one to determine the flux of solar $\nu_{e}$ on the earth, while the detection of solar neutrinos through the observation of the NC reaction allows one to determine the flux of all flavor neutrinos $\left(\nu_{e}, \nu_{\mu}\right.$ and $\left.\nu_{\tau}\right)$. It was shown in the SNO experiment that the flux of the solar $\nu_{e}$ was approximately three times smaller than the flux of $\nu_{e}, \nu_{\mu}$ and $\nu_{\tau}$. Thus, it was proved that solar $\nu_{e}$ 's on the way from the sun to the earth were transferred to $\nu_{\mu}$ and $\nu_{\tau}$.

In 2002-2004 model independent evidence of oscillations of reactor $\nu_{e}$ was obtained in the KamLAND reactor experiment [77] (Japan). In this experiment $\nu_{e}$ 's from 55 reactors at an average distance of about $170 \mathrm{~km}$ from the large KamLAND detector were recorded. It was found that the total number of $\bar{\nu}_{e}$ events was about 0.6 of the number of the expected events. A significant distortion of the $\bar{\nu}_{e}$ spectrum with respect to the expected spectrum was observed in the experiment.

Neutrino oscillations were observed also in the long-baseline accelerator K2K experiment [78] (the distance $L$ between the source and the detector was about $250 \mathrm{~km}$ ) and in the MINOS accelerator neutrino experiment [79] (with a distance $L$ of about $730 \mathrm{~km}$ ). These experiments fully confirmed the results obtained in the atmospheric Super-Kamiokande experiment.

Thus, neutrino oscillations were discovered. It was proven that neutrinos had small masses and that the flavor neutrinos $\nu_{e}, \nu_{\mu}, \nu_{\tau}$ were "mixed particles". The analysis of existing data, which we will briefly discuss in the next subsection, shows that existing neutrino oscillation data are well described if we assume the three-neutrino mixing.

\subsection{Present status of neutrino oscillations}

\section{Introduction}


The discovery of neutrino oscillations was a result of efforts of many physicists for many years. It required to build very large neutrino detectors (like SuperKamiokande, SNO, KamLAND and others) and to overcome severe background problems. Nevertheless, there were several "lucky circumstances" which made it possible to discover and investigate this phenomenon in some detail.

In the case of tree-neutrino mixing there are two independent masssquared differences $\Delta m_{23}^{2}$ and $\Delta m_{12}^{2}$. It was a "lucky circumstance" that both mass-squared differences could be reached in neutrino experiments: the first one in the atmospheric Super-Kamiokande experiment 48 and the second one in the long baseline KamLAND reactor experiment. The second "lucky circumstance" was the fact that the neutrino mixing angles $\theta_{23}$ and $\theta_{12}$ are large. As a result, effects of neutrino oscillations in the Super-Kamiokande and KamLAND experiments were large. This, of course, "simplified" the observation of neutrino oscillations in these experiments.

In this subsection we will briefly discuss the present status of the neutrino mixing and oscillations. We will consider the case of the three-neutrino mixing. "Mixed" flavor fields $\nu_{l L}(x)$, which enter into $\mathrm{CC}$ and $\mathrm{NC}$, are given in this case by the relations

$$
\nu_{l L}(x)=\sum_{i=1}^{3} U_{l i} \nu_{i L}(x) . \quad l-e, \mu, \tau
$$

Here $U$ is the $3 \times 3$ unitary PMNS mixing matrix and $\nu_{i}(x)$ is the field of neutrinos with mass $m_{i}$.

There is a lot of discussions in the literature on the methods of derivation from (176) of the observable probability for the transition between different types of neutrinos. Practically all methods give the same expression for the transition probability. We will stress here the physical principles on which oscillations are based and we will use the formalism which is similar to the formalism of the $K^{0} \rightleftarrows \bar{K}^{0}$ oscillations.

We know from neutrino oscillation experiments (see later) that the neutrino mass squared differences $\Delta m_{i k}^{2}=m_{k}^{2}-m_{i}^{2}$ are so small that the quantities $\frac{E}{\Delta m_{i k}^{2}}(E$ is the neutrino energy) are macroscopically large (about $10 \mathrm{~km}$

\footnotetext{
${ }^{48}$ Long-baseline accelerator experiments started at the time when indications (MINOS) and evidence $(\mathrm{K} 2 \mathrm{~K}, \mathrm{~T} 2 \mathrm{~K})$ of neutrino oscillations were obtained in the atmospheric neutrino experiments.
} 
for reactor neutrinos and about $100 \mathrm{~km}$ for accelerator neutrinos). As a result, differences between momenta of neutrinos with different masses produced in weak decays or reactions are much smaller than quantum mechanical uncertainties of momenta (determined by the Heisenberg uncertainty relation). Thus, production (and detection) of neutrinos with different masses can not be resolved and in CC weak processes together with a lepton $l^{+}$a flavor neutrino $\nu_{l}$, which is described by the coherent superposition

$$
\left|\nu_{l}\right\rangle=\sum_{i=1}^{3} U_{l i}^{*}\left|\nu_{i}\right\rangle,
$$

is produced (and detected).

We are interested in neutrino beams. Thus, the states $\left|\nu_{i}\right\rangle$ in (177) are states of neutrinos $\nu_{i}$ with mass $m_{i}$, helicity -1 , momentum $\vec{p}$ and energy $E_{i}=\sqrt{p^{2}+m_{i}^{2}} \simeq p+\frac{m_{i}^{2}}{2 p}$.

If at $t=0$ a flavor neutrino $\nu_{l}$ is produced, we have for the neutrino state in vacuum at $t>0$

$$
\left|\nu_{l}\right\rangle_{t}=e^{-i H_{0} t} \sum_{i}\left|\nu_{i}\right\rangle U_{l i}^{*}=\sum_{i}\left|\nu_{i}\right\rangle e^{-i E_{i} t} U_{l i}^{*}
$$

where $H_{0}$ is the free Hamiltonian. Neutrinos are detected via the observation of weak processes in which flavor neutrinos take part $\left(\nu_{l^{\prime}}+N \rightarrow l^{\prime}+X\right.$ etc $)$. Developing (178) over states $\left|\nu_{l^{\prime}}\right\rangle$ we find

$$
\left|\nu_{l}\right\rangle_{t}=\sum_{l^{\prime}}\left|\nu_{l^{\prime}}\right\rangle \sum_{i} U_{l^{\prime} i} e^{-i E_{i} t} U_{l i}^{*}
$$

If $m_{i}=m$, in this case $E_{i}=E, \sum_{i} U_{l^{\prime} i} U_{l i}^{*}=\delta_{l^{\prime} l}$ and $\left|\nu_{l}\right\rangle_{t}=e^{-i E t}\left|\nu_{l}\right\rangle$. Thus, if all neutrino masses are equal, the produced $\nu_{l}$ will always remain $\nu_{l}$. If neutrino masses are different, in this case the initial $\nu_{l}$ can be transferred into another flavor neutrino $\nu_{l^{\prime}}$. The probability of the transition $\nu_{l} \rightarrow \nu_{l^{\prime}}$ is given by the expression

$$
P\left(\nu_{l} \rightarrow \nu_{l^{\prime}}\right)=\left|\sum_{i} U_{l^{\prime} i} e^{-i E_{i} t} U_{l i}^{*}\right|^{2}
$$

Taking into account that for ultrarelativistic neutrinos $t \simeq L$, where $L$ is the distance between the neutrino source and the neutrino detector, and 
$E_{i}-E_{2}=\frac{\Delta m_{2 i}^{2} L}{2 E}$ we can rewrite expression (181) in the form

$$
P\left(\nu_{l} \rightarrow \nu_{l^{\prime}}\right)=\left|\delta_{l^{\prime} l}+\sum_{i \neq 2} U_{l^{\prime} i}\left(e^{-i \frac{\Delta m_{2 i}^{2} L}{2 E}}-1\right) U_{l i}^{*}\right|^{2} .
$$

It follows from this expression that the probability of the transition depends periodically on the parameter $\frac{L}{E}$. expression (181) describes neutrino oscillations in vacuum. It is clear from (178) and (179) that neutrino oscillations happen if the states of neutrinos with different masses gain different phases after the evolution of the neutrino beam during the time $t$ (at the distance $L)$.

The unitary $3 \times 3$ matrix $U$ is characterized by four parameters: three mixing angles $\theta_{12}, \theta_{23}, \theta_{13}$ and one phase $\delta$. In the case of three neutrino masses there are two independent mass-squared differences $\Delta m_{23}^{2}$ and $\Delta m_{12}^{2}$. Thus, in the general case the transition probability $P\left(\nu_{l} \rightarrow \nu_{l^{\prime}}\right)$ depends on six parameters.

It follows from the analysis of experimental data that two parameters are small:

$$
\frac{\Delta m_{12}^{2}}{\Delta m_{23}^{2}} \simeq \frac{1}{30}, \quad \sin ^{2} 2 \theta_{13}=0.092 \pm 0.016 \pm 0.005
$$

In the first (leading) approximation we can neglect contributions of these parameters to neutrino transition probabilities. In this approximation, a rather simple picture of neutrino oscillations emerges.

In the leading approximation in the atmospheric region of $\frac{L}{E}$ (for $\frac{\Delta m_{23}^{2} L}{2 E} \gtrsim$ 1) $\nu_{\mu} \rightleftarrows \nu_{\tau}$ oscillations take place. In this case, the $\nu_{\mu} \rightarrow \nu_{\mu}$ survival probability has the simple two-neutrino form

$$
P\left(\nu_{\mu} \rightarrow \nu_{\mu}\right) \simeq 1-P\left(\nu_{\mu} \rightarrow \nu_{\tau}\right) \simeq 1-\frac{1}{2} \sin ^{2} 2 \theta_{23}\left(1-\cos \Delta m_{23}^{2} \frac{L}{2 E}\right) .
$$

Thus, in the leading approximation neutrino oscillations in the atmospheric region are characterized by the parameters $\Delta m_{23}^{2}$ and $\sin ^{2} 2 \theta_{23}$.

In the KamLAND reactor region $\left(\frac{\Delta m_{12}^{2} L}{2 E} \gtrsim 1\right) \bar{\nu}_{e} \rightleftarrows \bar{\nu}_{\mu, \tau}$ take place. For the $\bar{\nu}_{e} \rightarrow \bar{\nu}_{e}$ survival probability we have

$$
P\left(\bar{\nu}_{e} \rightarrow \bar{\nu}_{e}\right)=1-\frac{1}{2} \sin ^{2} 2 \theta_{12}\left(1-\cos \Delta m_{12}^{2} \frac{L}{2 E}\right) .
$$

Thus, in the leading approximation, neutrino oscillations in the KamLAND reactor region are characterized by the parameters $\Delta m_{12}^{2}$ and $\sin ^{2} 2 \theta_{12}$. 
We also remark that in leading approximation the probability of the solar neutrinos to survive is given by the two-neutrino $\nu_{e} \rightarrow \nu_{e}$ survival probability in matter which depends on the parameters $\Delta m_{12}^{2}$ and $\sin ^{2} \theta_{12}$ and the electron number density.

The leading approximation gives the dominant contribution to the expressions for the neutrino transition probabilities. Until recently, in the analysis of neutrino oscillation data two-neutrino expressions (183) and (184) were used. Now with the improvement of the accuracy of the experiments threeneutrino transition probabilities are started to be used in the analysis of the data.

We will briefly discuss the results that were obtained in some neutrino oscillation experiments.

The SNO solar neutrino experiment [76].

The experiment was carried out in the Creighton mine (Sudbury, Canada) at a depth of $2092 \mathrm{~m}$. Solar neutrino were detected by a large heavy-water detector (1000 tons of $\mathrm{D}_{2} \mathrm{O}$ contained in an acrylic vessel of $12 \mathrm{~m}$ in diameter). The detector was equipped with 9456 photo-multipliers to detect light created by particles which are produced in neutrino interaction.

The high-energy ${ }^{8} B$ neutrinos were detected in the SNO experiment. An important feature of the SNO experiment was the observation of solar neutrinos via three different processes.

1. The CC process

$$
\nu_{e}+d \rightarrow e^{-}+p+p
$$

2. The NC process

$$
\nu_{x}+d \rightarrow \nu_{x}+p+n \quad(x=e, \mu, \tau)
$$

3. Elastic neutrino-electron scattering (ES)

$$
\nu_{x}+e \rightarrow \nu_{x}+e
$$

The detection of solar neutrinos through the observation of the NC reaction (186) allows one to determine the total flux of $\nu_{e}, \nu_{\mu}$ and $\nu_{\tau}$ on the earth. In the SNO experiment it was found

$$
\Phi_{\nu_{e, \mu, \tau}}^{N C}=\left(5.25 \pm 16_{-0.13}^{+0.11}\right) \cdot 10^{6} \mathrm{~cm}^{-2} \mathrm{~s}^{-1} .
$$


The total flux of all active neutrinos on the earth must be equal to the total flux of $\nu_{e}$ emitted by the sun (if there are no transitions of $\nu_{e}$ into sterile neutrinos). The flux measured by SNO is in agreement with the total flux of $\nu_{e}$ predicted by the Standard Solar Model:

$$
\Phi_{\nu_{e}}^{S S M}=(4.85 \pm 0.58) \cdot 10^{6} \mathrm{~cm}^{-2} \mathrm{~s}^{-1} .
$$

The detection of the solar neutrinos via reaction (185) allows one to determine the total flux of $\nu_{e}$ on the earth. It was found in the SNO experiments that the total flux of $\nu_{e}$ was about three times smaller than the total flux of all active neutrinos.

From the ratio of the fluxes of $\nu_{e}$ and $\nu_{e}, \nu_{\mu}$ and $\nu_{\tau}$ the $\nu_{e}$ survival probability can be determined. It was shown in the SNO experiment that in the high-energy ${ }^{8} B$ region the $\nu_{e}$ survival probability did not depend on the neutrino energy and was equal to

$$
\frac{\Phi_{\nu_{e}}^{C C}}{\Phi_{\nu_{e, \mu, \tau}}^{N C}}=P\left(\nu_{e} \rightarrow \nu_{e}\right)=0.317 \pm 0.016 \pm 0.009
$$

Thus, it was proved in a direct, model independent way that solar $\nu_{e}$ on the way to the earth are transformed into $\nu_{\mu}$ and $\nu_{\tau}$.

The KamLAND reactor neutrino experiment [77]

The KamLAND detector is situated in the Kamioka mine (Japan) at a depth of about $1 \mathrm{~km}$. The neutrino target is a 1 kiloton liquid scintillator which is contained in a 13 m-diameter transparent nylon balloon suspended in $1800 \mathrm{~m}^{3}$ non-scintillating buffer oil. The balloon and buffer oil are contained in an $18 \mathrm{~m}$-diameter stainless-steel vessel. On the inner surface of the vessel 1879 photomultipliers are mounted.

In the KamLAND experiment $\bar{\nu}_{e}$ from 55 reactors situated at distances of $175 \pm 35 \mathrm{~km}$ from the Kamioka mine are detected.

Reactor $\bar{\nu}_{e}$ 's are detected in the KamLAND experiment through the observation of the process

$$
\bar{\nu}_{e}+p \rightarrow e^{+}+n
$$

The signature of the neutrino event is a coincidence between two $\gamma$-quanta produced in the annihilation of a positron (prompt signal) and $\mathrm{a} \simeq 2.2 \mathrm{MeV}$ $\gamma$-quantum produced by a neutron capture in the process $n+p \rightarrow d+\gamma$ (delayed signal). 
The average energy of the reactor antineutrinos is $3.6 \mathrm{MeV}$. For such energies, distances of about $100 \mathrm{~km}$ are appropriate to study neutrino oscillations driven by the solar neutrino mass-squared difference $\Delta m_{12}^{2}$.

From March 2002 to May 2007 in the KamLAND experiment 1609 neutrino events were observed. The expected number of neutrino events (if there are no neutrino oscillations) is $2179 \pm 89$. Thus, it was observed in the experiment that $\bar{\nu}_{e}$ disappeared on the way from the reactors to the detector.

As the $\bar{\nu}_{e}$ survival probability depends on the neutrino energy we must expect that the detected spectrum of $\bar{\nu}_{e}$ is different from the spectrum produced by a reactor. In fact in the KamLAND experiment a significant distortion of the initial antineutrino spectrum is observed.

The data of the experiment are well described if we assume that twoneutrino oscillations take place. For the neutrino oscillation parameters it was found

$$
\Delta m_{12}^{2}=\left(7.66_{-0.22}^{+0.20}\right) \cdot 10^{-5} \mathrm{eV}^{2}, \quad \tan ^{2} 2 \theta_{12}=0.52_{-0.10}^{+0.16}
$$

From the three-neutrino analysis of all solar neutrino data and the data of the KamLand reactor experiment for the neutrino oscillation parameters the following values were obtained

$$
\Delta m_{12}^{2}=\left(7.41_{-0.19}^{+0.21}\right) \cdot 10^{-5} \mathrm{eV}^{2}, \quad \tan ^{2} \theta_{12}=0.446_{0.029}^{+0.030}, \quad \sin ^{2} \theta_{13}<0.053 .
$$

\section{Super-Kamiokande atmospheric neutrino experiment [75]}

In the Super-Kamiokande atmospheric neutrino experiment the first modelindependent evidence in favor of neutrino oscillations was obtained (1998). The Super-Kamiokande detector is situated in the same Kamioka mine as the KamLAND detector. It consists of two optically separated water-Cherenkov cylindrical detectors with a total mass of 50 kilotons of water. The inner detector with 11146 photomultipliers has a radius of $16.9 \mathrm{~m}$ and a height of $36.2 \mathrm{~m}$. The outer detector is a veto detector. It allows to reject cosmic ray muons. The fiducial mass of the detector is 22.5 kilotons.

In the Super-Kamiokande experiment atmospheric neutrinos in a wide range of energies from about $100 \mathrm{MeV}$ to about $10 \mathrm{TeV}$ are detected . Atmospheric $\nu_{\mu}\left(\bar{\nu}_{\mu}\right)$ and $\nu_{e}\left(\bar{\nu}_{e}\right)$ are detected through the observation of $\mu^{-}\left(\mu^{+}\right)$ and $e^{-}\left(e^{+}\right)$produced in the processes

$$
\nu_{\mu}\left(\bar{\nu}_{\mu}\right)+N \rightarrow \mu^{-}\left(\mu^{+}\right)+X, \quad \nu_{e}\left(\bar{\nu}_{e}\right)+N \rightarrow e^{-}\left(e^{+}\right)+X .
$$


For the study of neutrino oscillations it is important to distinguish electrons and muons produced in the processes (194). In the Super-Kamiokande experiment leptons are observed through the detection of the Cherenkov radiation. The shapes of the Cherenkov rings of electrons and muons are completely different (in the case of electrons the Cherenkov rings exhibit a more diffuse light than in the muon case). The probability of a misidentification of electrons and muons is below $2 \%$.

A model-independent evidence of neutrino oscillations was obtained by the Super-Kamiokande Collaboration through the investigation of the zenithangle dependence of the electron and muon events. The zenith angle $\theta$ is determined in such a way that neutrinos going vertically downward have $\theta=0$ and neutrinos coming vertically upward through the earth have $\theta=\pi$. At neutrino energies $E \gtrsim 1 \mathrm{GeV}$ the fluxes of muon and electron neutrinos are symmetric under the change $\theta \rightarrow \pi-\theta$. Thus, if there are no neutrino oscillations in this energy region the numbers of electron and muon events must satisfy the relation

$$
N_{l}(\cos \theta)=N_{l}(-\cos \theta) \quad l=e, \mu .
$$

In the Super-Kamikande experiment a large violation of this relation for high energy muon events was established (a significant deficit of upward-going muons was observed). The number of electron events satisfies the relation (195).

This result can naturally be explained by the disappearance of muon neutrinos due to neutrino oscillations. The probability for $\nu_{\mu}$ to survive depends on the distance between the neutrino source and the neutrino detector. Downward going neutrinos $(\theta \simeq 0)$ pass a distance of about $20 \mathrm{~km}$. On the other side upward going neutrinos $(\theta \simeq \pi)$ pass a distance of about 13000 $\mathrm{km}$ (earth diameter). The measurement of the dependence of the numbers of the electron and muon events on the zenith angle $\theta$ allows one to span the whole region of distances from about $20 \mathrm{~km}$ to about $13000 \mathrm{~km}$.

From the data of the Super-Kamiokande experiment for high-energy electron events was found

$$
\left(\frac{U}{D}\right)_{e}=0.961_{-0.079}^{+0.086} \pm 0.016 .
$$

For high-energy muon events the value

$$
\left(\frac{U}{D}\right)_{\mu}=0.551_{-0.033}^{+0.035} \pm 0.004 \text {. }
$$


was obtained. Here $U$ is the total number of upward going leptons $(-1<$ $\cos \theta<-0.2)$ and $D$ is the total number of downward going leptons $(0.2<$ $\cos \theta<1)$.

The data of the Super-Kamiokande atmospheric neutrino experiment are perfectly described if we assume that $\nu_{\mu}$ 's disappear mainly due to $\nu_{\mu} \rightleftarrows \nu_{\tau}$ oscillations. From the three-neutrino analysis of the data for neutrino oscillation parameters in the case of normal (inverted) neutrino mass spectrum it was found

$$
\begin{gathered}
1.9(1.7) \cdot 10^{-3} \mathrm{eV}^{2} \leq \Delta m_{23}^{2} \leq 2.6(2.7) \cdot 10^{-3} \mathrm{eV}^{2} \\
0.407 \leq \sin ^{2} \theta_{23} \leq 0.583, \quad \sin ^{2} \theta_{13}<0.04(0.09)
\end{gathered}
$$

The result of the Super-Kamiokande atmospheric neutrino experiment was confirmed by

the long-baseline accelerator neutrino experiments $\mathrm{K} 2 \mathrm{~K}$ and MINOS [78, 79]

In the MINOS experiment, muon neutrinos produced at the Fermilab Main Injector facility are detected. The MINOS data were obtained with neutrinos mostly with energies in the range $1 \leq E \leq 5 \mathrm{GeV}$.

There are two identical neutrino detectors in the experiment. The near detector with a mass of 1 kiloton is at a distance about $1 \mathrm{~km}$ from the target of the accelerator and about $100 \mathrm{~m}$ underground. The far detector with a mass of 5.4 kilotons is at a distance of $735 \mathrm{~km}$ from the target in the Sudan mine (about $700 \mathrm{~m}$ underground).

Muon neutrinos (antineutrinos) are detected in the experiment via the observation of the process

$$
\nu_{\mu}\left(\bar{\nu}_{\mu}\right)+\mathrm{Fe} \rightarrow \mu^{-}\left(\mu^{+}\right)+\mathrm{X}
$$

The neutrino energy is given by the sum of the muon energy and the energy of the hadronic shower.

In the near detector the initial neutrino spectrum is measured. This measurement allows to predict the expected spectrum of the muon neutrinos in the far detector in the case if there were no neutrino oscillations. A strong distortion of the spectrum of $\nu_{\mu}\left(\bar{\nu}_{\mu}\right)$ in the far detector was observed in the MINOS experiment.

From the two-neutrino analysis of the $\nu_{\mu}$ data for the neutrino oscillations parameters the following values were obtained

$$
\Delta m_{23}^{2}=\left(2.32_{-0.08}^{+0.12}\right) \cdot 10^{-3} \mathrm{eV}^{2}, \quad \sin ^{2} 2 \theta_{23}>0.90 .
$$




\section{Indications in favor of nonzero $\theta_{13}$}

The value of the mixing angle $\theta_{13}$ is extremely important for the future of the neutrino physics. If this angle is not equal to zero (and relatively large) in this case it will be possible to observe such a fundamental effect of the threeneutrino mixing as $C P$ violation in the lepton sector. Another problem, the solution of which requires nonzero $\theta_{13}$, is the problem of the neutrino mass spectrum. In the case of the three massive neutrinos with two mass-squared differences $\Delta m_{23}^{2}$ and $\Delta m_{12}^{2}$ two neutrino mass spectra are possible

1. Normal spectrum

$$
m_{1}<m_{2}<m_{3} ; \quad \Delta m_{12}^{2} \ll \Delta m_{23}^{2}
$$

2. Inverted spectrum

$$
m_{3}<m_{1}<m_{2} ; \quad \Delta m_{12}^{2} \ll\left|\Delta m_{13}^{2}\right|
$$

Let us notice that in order to have the same notation $\Delta m_{12}^{2}$ for the solar masssquared difference for both spectra the neutrino masses are usually labeled differently in the cases of the normal and inverted neutrino mass spectra. In the case of the normal spectrum $\Delta m_{23}^{2}>0$ and in the case of the inverted spectrum $\Delta m_{13}^{2}<0$.

For many years only an upper bound on the parameter $\sin ^{2} \theta_{13}$ existed. This bound was obtained from the analysis of the data of the CHOOZ reactor experiment [80].

In the CHOOZ experiment the detector ( 5 tons of Gd-loaded liquid scintillator) was at a distance of about $1 \mathrm{~km}$ from each of the two reactors of the CHOOZ power station (8.5 GWth). The detector had $300 \mathrm{~m}$ water equivalent of rock overburden which reduced the cosmic muon flux. The antineutrinos were detected through the observation of the classical reaction

$$
\bar{\nu}_{e}+p \rightarrow e^{+}+n \text {. }
$$

For the ratio $R$ of the total number of detected $\bar{\nu}_{e}$ events to the number of the expected events it was found the value

$$
R=1.01 \pm 2.8 \% \text { (stat) } \pm \pm 2.7 \% \text { (syst). }
$$

The data of the experiment was analyzed in the framework of two-neutrino oscillations with the $\bar{\nu}_{e}$-survival probability given by the expression

$$
P\left(\bar{\nu}_{e} \rightarrow \bar{\nu}_{e}\right)=1-\frac{1}{2} \sin ^{2} 2 \theta_{13}\left(1-\cos \frac{\Delta m_{23}^{2} L}{2 E}\right)
$$


From the data of the CHOOZ experiment the following upper bound

$$
\sin ^{2} 2 \theta_{13} \leq 0.16
$$

was obtained.

In a new long baseline T2K neutrino experiment [81] an indication in favor of nonzero $\theta_{13}$ was obtained. In this experiment muon neutrinos produced at the J-PARC accelerator in Japan are detected at a distance of $295 \mathrm{~km}$ in the water-Cherenkov Super-Kamiokande detector. The T2K experiment is the first off-axis neutrino experiment: the angle between the direction to the detector and the flight direction of the parent $\pi^{+}$'s is equal to $2^{\circ}$. This allows one to obtain a narrow-band neutrino beam with a maximal intensity at the energy $E \simeq 0.6 \mathrm{GeV}$ which corresponds at the distance of $L=295$ $\mathrm{km}$ to the first oscillation maximum $\left(E_{0}=\frac{2.54}{\pi} \Delta m_{23}^{2} L\right)$.

At a distance of about $280 \mathrm{~m}$ from the target there are several near detectors which are used for the measurement of the neutrino spectrum and flux and for the measurement of cross sections of different CC and NC processes.

The initial beam (from decays of pions and kaons) is a beam of $\nu_{\mu}$ 's with a small (about $0.4 \%$ ) admixture of $\nu_{e}$ 's. The search for electrons in the Super-Kamiokande detector due to $\nu_{\mu} \rightarrow \nu_{e}$ transitions was performed. Six $\nu_{e}$ events were observed in the experiment. The expected number of electron events (without neutrino oscillations) is equal $1.5 \pm 0.3$. From the analysis of the data for the normal neutrino mass spectrum it was found:

$$
0.03<\sin ^{2} 2 \theta_{13}<0.28(90 \% \mathrm{CL}) \quad \text { best fit }: \sin ^{2} 2 \theta_{13}=0.11 \text {. }
$$

For the inverted neutrino mass spectrum it was found

$$
0.04<\sin ^{2} 2 \theta_{13}<0.34(90 \% \mathrm{CL}) \quad \text { best fit }: \sin ^{2} 2 \theta_{13}=0.14 \text {. }
$$

A similar experiment was performed by the MINOS collaboration. In this experiment for the normal (inverted) neutrino mass spectrum the following best fit value was found

$$
2 \sin ^{2}\left(\theta_{23}\right) \sin ^{2}\left(2 \theta_{13}\right)=0.041_{-0.031}^{+0.047}\left(0.079_{-0.053}^{+0.071}\right)
$$

The Double Chooz collaboration presented first indication in favor of reactor $\bar{\nu}_{e}$ 's disappearence [84]. For the ratio of the observed and predicted $\bar{\nu}_{e}$ events the value $0.944 \pm 0.016 \pm 0.040$ was found. At $90 \% C L$ it was obtained $0.015 \sin ^{2} 2 \theta_{13}<0.16$. 


\section{Measurement of nonzero $\theta_{13}$; the Daya Bay and RENO experi- ments}

Recently results of the Daya Bay reactor neutrino experiment were published [85]. In this experiment reactor antineutrinos from six reactors (the thermal power of each reactor is $2.9 \mathrm{Gw}$ ) were detected by three near detectors (distances $470 \mathrm{~m}$ and $570 \mathrm{~m}$ ) and three far detectors $(1648 \mathrm{~m})$. Antineutrinos are detected via observation of the classical reaction

$$
\bar{\nu}_{e}+p \rightarrow e^{+}+n \text {. }
$$

Each detector contains the 20-ton Gd-loaded liquid scintillator. During 55 days of the data taking 10416 (80376) $\bar{\nu}_{e}$-events were observed in far (near) detectors. The number of $\bar{\nu}_{e}$ events in the far detectors can be predicted (assuming that there are no neutrino oscillations) on the basis of measurements performed in the near detectors. For the ratio $R$ of the total numbers of the observed and predicted events the following value was obtained

$$
R=0.940 \pm 0.011 \pm 0.004 .
$$

The probability of $\bar{\nu}_{e}$ to survive is given by the following expression

$$
P\left(\bar{\nu}_{e} \rightarrow \bar{\nu}_{e}\right)=1-\sin ^{2} 2 \theta_{13} \sin ^{2} 1.267 \frac{\Delta m_{23}^{2} L}{E},
$$

where $\Delta m_{23}^{2}$ is the neutrino mass-squared difference in $\mathrm{eV}^{2}, L$ is the sourcedetector distance in $\mathrm{m}$ and $E$ is the antineutrino energy in $\mathrm{MeV}$. From $\chi^{2}$ analysis of the data it was found that

$$
\sin ^{2} 2 \theta_{13}=0.092 \pm 0.016 \pm 0.005 .
$$

Thus, zero value of the parameter $\sin ^{2} 2 \theta_{13}$ is excluded at the level $5.2 \sigma$.

The value of the parameter $\sin ^{2} 2 \theta_{13}$ obtained in the similar two-detectors reactor RENO experiment [86] is in agreemment with (212). In this experiment it was found

$$
\sin ^{2} 2 \theta_{13}=0.113+0.013+0.019
$$

\section{Conclusion}

We followed here some basic facts of the history of neutrinos, unique particles which brought three Nobel Prizes to elementary particle physics. The 
neutrino history is very interesting, instructive, sometimes dramatic. There were many wrong experiments in the history of the neutrino (like $\beta$-decay experiments on electron-neutrino correlation which favored $S, T$ couplings in the fifties, first experiment on the search for $\pi \rightarrow e \nu$-decay, experiments from which the existence of a heavy neutrino with a mass of $17 \mathrm{keV}$ followed at the beginning of the nineties, etc.) and wrong common opinions lasting for many years (like the general opinion that the neutrino is an undetectable particle in the thirties and forties, the general opinion that the neutrino is a massless particle in the fifties and sixties, etc.).

The neutrino hypothesis was born in 1930 in an attempt to save the law of conservation of energy and momentum ("I have hit upon a desperate remedy to save the "exchange theorem" of statistics and the law of conservation of energy. Namely, the possibility that in the nuclei there could exist electrically neutral particles, which I will call neutrons, that have spin $1 / 2$ and obey the exclusion principle and that further differ from light quanta in that they do not travel with the velocity of light." Pauli's letter). The assumption of the existence of the neutrino allowed Fermi to build a phenomenological theory of the $\beta$-decay of nuclei and other weak processes which could describe a lot of experimental data. However, it took more that twenty years to prove by a direct experiment that neutrinos exist.

There are two unique properties of neutrinos which determine their importance and their problems:

1. Neutrinos have only weak interaction.

2. Neutrinos have very small masses.

Since neutrinos have only weak interaction, cross sections of interaction of neutrinos with nucleons are extremely small. This means that it is necessary to develop special methods of neutrino detection (large detectors which often are situated in underground laboratories in order to prevent cosmic ray background etc). However, when methods of neutrino detection were developed, neutrinos became a unique instrument in the study of the sun (solar neutrino experiments allow us to obtain information about the central invisible region of the sun in which solar energy is produced in thermonuclear reactions), in the investigation of a mechanism of the Supernova explosion 49 (99\% of the

\footnotetext{
${ }^{49}$ On February 23, 1987 for the first time antineutrinos from Supernova SN1987A in the Large Magellanic Cloud were detected by Kamiokande, IMB and Baksan detectors.
} 
energy produced in a Supernova explosion is emitted in neutrinos), in establishing the quark structure of a nucleon (through the study of the deep inelastic processes $\nu_{\mu}\left(\bar{\nu}_{\mu}\right)+N \rightarrow \mu^{-}\left(\mu^{+}+X\right)$, etc.

In the fifties the majority of physicists believed that the neutrino was a massless particle. This was an important, constructive assumption (in spite of that it was wrong). The theory of the two-component neutrino, which was based on this assumption, inspired the creation of the phenomenological $V-A$ theory and later became part of the Standard Model of the electroweak interaction.

Neutrino masses are very small and it is very difficult to observe effects of neutrino masses in the $\beta$-decay and in other weak processes. However, small neutrino masses and, correspondingly, mass-squared differences make it possible the production (and detection) of the coherent flavor neutrino states(states of $\nu_{e}, \nu_{\mu}, \nu_{\tau}$ ) and quantum-mechanical periodical transitions between different flavor neutrino states (neutrino oscillations). The observation of neutrino oscillations at large (macroscopic) distances allowed one to resolve small neutrino mass-squared differences.

The discovery of neutrino oscillations signifies a new era in neutrino physics, the era of investigation of neutrino properties. From the analysis

of the existing neutrino oscillation data two mass-squared differences $\Delta m_{23}^{2}$ and $\Delta m_{12}^{2}$ and two mixing parameters $\sin ^{2} \theta_{23}$ and $\tan ^{2} \theta_{12}$ are determined with accuracies in the range $(3-12) \%$. The results of the first measurement of the parameter $\sin ^{2} 2 \theta_{13}$ was recently announced by the Daya Bay collaboration.

One of the most urgent problems which will be addressed in the next neutrino oscillation experiments are

\section{CP violation in the lepton sector.}

\section{Character of the neutrino mass spectrum (normal or inverted?)}

Relatively "large" value of the angle $\theta_{13}$ obtained in the Daya Bay and other experiments open the way for the investigation of these problems in the near years.

One of the most important problems of the physics of massive and mixed neutrinos is the problem of the nature of neutrinos with definite masses $\nu_{i}$.

In 2002 The Nobel Prize was awarded to R. Davis (solar neutrinos) and M. Koshiba (supernova neutrinos) "for pioneering contributions to astrophysics, in particular for the detection of cosmic neutrinos". 


\section{Are neutrinos with define masses Dirac particles possessing con- served lepton number or truly neutral Majorana particles?}

The answer to this fundamental question can be obtained in experiments on the search for neutrinoless double $\beta$-decay ( $0 \nu \beta \beta$-decay) of some even-even nuclei

$$
(A, Z) \rightarrow(A, Z+2)+e^{-}+e^{-} .
$$

This process is allowed only if the total lepton number is violated. If massive neutrinos are Majorana particles, $0 \nu \beta \beta$-decay (214) is the second order process in the Fermi constant with the exchange of the virtual neutrinos between neutron-proton-electron vertices. The matrix element of the process is proportional to the effective Majorana mass

$$
m_{\beta \beta}=\sum_{i} U_{e i}^{2} m_{i}
$$

Many experiments on the search for the $0 \nu \beta \beta$-decay of different nuclei were performed. No evidence in favor of the process was obtained. The most stringent lower bound for the half-life of the process was obtained in the experiment [82] on the search for the decay

$$
{ }^{76} \mathrm{Ge} \rightarrow{ }^{76} \mathrm{Se}+\mathrm{e}^{-}+\mathrm{e}^{-}
$$

In this experiment the following lower bound was obtained

$$
T_{1 / 2}^{0 \nu}\left({ }^{76} \mathrm{Ge}\right)>1.9 \cdot 10^{25} \text { years }
$$

Taking into account different calculations of the nuclear matrix element from this bound it can be found

$$
\left|m_{\beta \beta}\right|<(0.20-0.32) \mathrm{eV} .
$$

Future experiments on the search for the $0 \nu \beta \beta$-decay will be sensitive to the value

$$
\left|m_{\beta \beta}\right| \simeq \text { (a few) } 10^{-2} \mathrm{eV}
$$

and can probe the Majorana nature of $\nu_{i}$ in the case of the inverted hierarchy of the neutrino masses

$$
m_{3} \ll m_{1}<m_{2} .
$$

Another fundamental problem of the physics of massive and mixed neutrinos is 


\section{What are the absolute value of the neutrino masse?}

From the data of neutrino oscillation experiments only the mass-squared differences can be determined. The absolute value of the "average" neutrino mass $m_{\beta}$ can be inferred from the investigation of $\beta$-spectra. From the data of the latest MAINZ and Troitsk tritium experiments the following bound was obtained

$$
m_{\beta}<2.3 \mathrm{eV},
$$

where $m_{\beta}=\sqrt{\sum_{i}\left|U_{e 1}\right|^{2} m_{i}^{2}}$ is the "average" neutrino mass. The future tritium experiment KATRIN will be sensitive to

$$
m_{\beta}<0.2 \mathrm{eV}
$$

Precision modern cosmology became an important source of information about absolute values of neutrino masses. Different cosmological observables (Large Scale Structure of the Universe, Gravitational Lensing of Galaxies, Primordial Cosmic Microwave Background, etc.) are sensitive to the sum of the neutrino masses $\sum_{i} m_{i}$. From the existing data the following bounds were obtained

$$
\sum_{i} m_{i}<(0.2-1.3) \mathrm{eV}
$$

It is expected that future cosmological observables will be sensitive to the sum of neutrino masses in the range

$$
\sum_{i} m_{i} \simeq(0.05-0.6) \mathrm{eV}
$$

These future measurements, apparently, will probe the inverted neutrino mass hierarchy (216) $\left(\sum_{i} m_{i} \simeq 0.1 \mathrm{eV}\right)$ and even the normal neutrino mass hierarchy

$$
m_{1} \ll m_{2} \ll m_{3}, \quad \sum_{i} m_{i} \simeq 0.05 \mathrm{eV} .
$$

The next question which needs to be answered is

\section{How many neutrinos with definite masses exist in nature?}

We considered the minimal scheme with three flavor neutrinos $\left(\nu_{e}, \nu_{\mu}, \nu_{\tau}\right)$ and, correspondingly, three massive neutrinos $\left(\nu_{1}, \nu_{2}, \nu_{3}\right)$. However, the number of massive light neutrinos can be more than three. In this case flavor neutrinos could oscillate into sterile states $\nu_{s}$, which do not have the standard weak interaction. 
For many years there was an indication in favor of more than three light neutrinos with definite masses obtained in a short-baseline LSND experiment [83. In this experiment the $\bar{\nu}_{\mu} \rightarrow \bar{\nu}_{e}$ transition driven by $\Delta m^{2} \simeq 1 \mathrm{eV}^{2}$, which is much larger than the atmospheric mass-squared difference, was observed. Some indications in favor of more than three massive neutrinos were also obtained in the MiniBooNE and reactor experiments. New short-baseline accelerator and reactor experiments are urgently needed. Such experiments are now at preparation.

There are other questions connected with neutrinos which now are being actively discussed in the literature (neutrino magnetic moments, nonstandard interaction of neutrinos, etc.).

An explanation of small neutrino masses requires a new, beyond the Standard Model (Higgs) mechanism of neutrino mass generation. But what mechanism, what kind of new physics is required to explain small neutrino masses and peculiar neutrino mixing? This is at the moment an open question. Several new mechanisms of neutrino mass generation were proposed in the literature. Apparently, the most plausible mechanism is the seesaw mechanism of the neutrino mass generation [71].

The seesaw mechanism is based on the assumption that the total lepton number $L$ is violated at a large scale $M$. From the seesaw mechanism the following general consequences follow

1. Neutrinos with definite masses $\nu_{i}$ are Majorana particles.

2. Neutrino masses are given by the seesaw formula

$$
m_{i} \simeq y_{i} \frac{v^{2}}{M}
$$

where $y_{i}$ is a dimensionless Yukawa constant and the parameter $v \simeq$ $250 \mathrm{GeV}$ characterizes the scale of the violation of the electroweak symmetry.

The scale of the violation of the lepton number $M$ depends on the Yukawa constants $y_{i}$ which are unknown. Different options are discussed in the literature. If $y_{i} v \simeq m_{i}^{f}$, where $m_{i}^{f}$ is the mass of a quark or a lepton, in this case $M \simeq\left(10^{14}-10^{15}\right) \mathrm{GeV}$ and the only implication of the violation of the lepton number in the region of the electroweak energies are Majorana 
neutrino masses 50

If $M \simeq 1 \mathrm{TeV}$ in this case $y_{i} v \ll m_{i}^{f}$. Existence of Majorana particles with masses $\simeq 1 \mathrm{TeV}$ could be revealed through (see [88])

1. an additional contribution to the matrix elements of the neutrinoless double $\beta$-decay;

2. observation of the lepton number violating processes of production of pairs of the same sign leptons in proton-proton collisions at LHC.

In order to reveal the true nature of neutrino masses and mixing many new investigations must be performed. The history of neutrinos, unique particles, is continuing. There are no doubts that new surprises and discoveries (and, possibly, Nobel Prizes) are ahead.

I am thankful to the theory group of TRIUMF for the hospitality. It is a pleasure for me to thank $W$. Potzel for careful reading of the paper and numerous remarks and suggestions.

\section{References}

[1] C. D. Ellis and W.A. Wooster, Proc. Roy. Soc. A117 (1927) 109.

[2] J. Chadwick, Nature 193 (1932) 312.

[3] W. Heisenberg, Z.Phys.77 (1932)1; Z.Phys.78 (1932)156.

[4] E. Majorana, Z.Phys.82 (1933)137.

[5] D. Ivanenko, Nature 129 (1932)798.

[6] E. Fermi, Zeitschr. f. Phys. 88 (1934) 161.

[7] F. Perrin, Comptes Rendus 197 (1933) 1625.

[8] G. Gamow and E. Teller, Phys. Rev. 49 (1936) 895.

[9] H.Bethe and R.Peierls, Nature 133 (1934) 532.

\footnotetext{
${ }^{50}$ Let us notice that $\mathrm{CP}$-violating decays of heavy Majorana particles, the seesaw partners of Majorana neutrinos, in the early Universe are commonly considered as a plausible source of the baryon asymmetry of the Universe [87.
} 
[10] B. Pontecorvo, Report PD-205, Chalk River Laboratory, 1946.

[11] B.T. Cleveland et al. Astrophys. J. 496 (1998) 505.

[12] B. Pontecorvo, Phys. Rev.72 (1947) 246.

[13] G. Puppi, Nuovo Cimento 5 (1948) 587.

[14] O. Klein, Nature 161 (1948) 897.

[15] C. N. Yang and J. Tiomno, Phys. Rev.79 (1950) 495.

[16] T. D. Lee and C. N. Yang, Phys. Rev. 104 (1956) 254.

[17] C. S. Wu et al., Phys. Rev. 105 (1957) 1413.

[18] R. L. Garwin, L. M. Lederman and W. Weinrich, Phys. Rev. 105 (1957) 1415.

[19] L. D. Landau, Nucl. Phys. 3 (1957) 127.

[20] T. D. Lee and C. N. Yang, Phys. Rev. 105 (1957) 1671.

[21] A. Salam, Nuovo Cim. 5 (1957) 299.

[22] H. Weil, Z. Physik 56 (1929) 330.

[23] W. Pauli, Handbuch der Physik, Springer Verlag, Berlin v.24 (1933) 226-227.

[24] E. Majorana, Nuovo Cimento 5 (1937) 171.

[25] M. Goldhaber, L. Grodzins and A. W. Sunyar, Phys. Rev. 109 (1958) 1015.

[26] R. P. Feynman and M. Gell-Mann, Phys. Rev. 109 (1958) 193.

[27] E. C. G. Sudarshan and R. E. Marshak, Phys. Rev. 109 (1958) 1860.

[28] S.S.Gerstein and Ja.B Zeldovich, Sov. Phys. JETP 2(1956)576.

[29] H.L. Anderson and C. Lattes, Nuovo Cimento 6 (1957) 1356.

[30] T. Fazzini, G. Fidecaro et al., Phys. Rev. Lett. 1 (1958) 247. 
[31] F. Reines, H.S. Gurr and H.W. Sobel, Phys. Rev. Lett. 37 (1976) 315.

[32] N. Cabibbo, Phys. Rev. Lett. 10 (1963) 531.

[33] O. Klein, Proc. Symp. on Les Nouvelles Theories de la Physique, Warsaw, 1938 (Institut International de Coop-ration Intellectuelle, Paris, 1939), p.6.

[34] F. Reines and C. L. Cowan, Phys. Rev. 92 (1953) 830; F. Reines and C. L. Cowan, Nature 178 (1956) 446; F. Reines and C. L. Cowan, Phys. Rev. 113 (1959) 273.

[35] R. Davis, Bull. Am. Phys. Soc., Washington meeting, 1959.

[36] B. Pontecorvo, Journal de Physique 43 N. 12 (1959) p.C8-221.

[37] G. Danby, J.-M. Gaillard, K. Goulianos, L.M. Lederman, N. Mistry, M. Schwartz and J. Steinberger, Phys. Rev. Lett. 9 (1962) 36.

[38] G. Feinberg, Phys. Rev. 110 (1958) 1482.

[39] B. Pontecorvo, Sov. Phys. JETP 10 (1960) 1236.

[40] K. Nakamura et al. (Particle Data Group), J. Phys. G37G 37 (2010) 075021.

[41] S. L. Glashow, J. Iliopoulos and L. Maiani, Phys. Rev. D2 (1970) 1258.

[42] S. M. Bilenky, B. Pontecorvo, Phys. Rep. 41 (1978) 225.

[43] M. L. Perl et al., Phys. Rev. Lett. 35, 1489 (1975).

[44] K. Kodama et al. (DONUT Collaboration), Physics Letters B 504 (2001) 218.

[45] M. Kobayashi and T. Maskawa, Progress of Theoretical Physics 49 (2) (1973) 652 .

[46] K. Nakamura et al. (Particle Data Group), J. Phys. G37G 37 (2010) 075021.

[47] S. Weinberg, Phys. Rev. Lett. 19 (1967) 1264. 
[48] A. Salam, Proc. of the Eighth Nobel Symposium (ed. N. Svartholm, Wiley-Interscience, New York (1968).

[49] S. Chatrchyan et al., Phys. Lett. B716 (2012) 30.

[50] G. Aad et al., Phys. Lett. B 716 (2012) 1.

[51] S. L. Glashow, Nucl. Phys. 22 (1961) 579.

[52] G. 't Hooft, Nucl. Phys. B35 (1971) 1967.

[53] C. N. Yang and R. Mills, Phys. Rev.96 (1954) 191

[54] F. J. Hasert et al., Phys. Lett. B46 (1973) 138.

[55] G.P.Zeller et al., Phys. Rev. Lett. 88 (2002) 091802.

[56] M. Gell-Mann and A. Pais, Phys. Rev. 97 (1955) 1387.

[57] B. Pontecorvo, J.Exptl. Theoret. Phys. 33 (1957) 549 [Sov. Phys. JETP 6 (1958) 429].

[58] B. Pontecorvo, J.Exptl. Theoret. Phys. 34 (1958) 247 [Sov. Phys. JETP 7 (1958) 172].

[59] B. Pontecorvo, J. Exptl. Theoret. Phys. 53 (1967) 1717. [Sov. Phys. JETP 26 (1968) 984].

[60] R. Davis et al.,Proc. Conf. "Neutrino 72", Hungary, vol.I p. 29, (1972).

[61] V. Gribov and B. Pontecorvo, Phys. Lett. B28 (1969) 493.

[62] J. Bahcall and S. Frautschi, Phys. Lett. 29 (1969) 623.

[63] S.M. Bilenky and B. Pontecorvo, Phys. Lett. B61 (1976) 248; Yad. Fiz. 3 (1976) 603 .

[64] H. Fritzsch and P. Minkowski, Phys. Lett. B62 (1976) 72.

[65] S. Eliezer and A. Swift, Nucl.Phys. B105 (1976) 45.

[66] Z. Maki, M. Nakagava and S. Sakata, Prog. Theor. Phys. 28 (1962) 870.

[67] S.M. Bilenky and B. Pontecorvo, Phys. Rep. 41 (1978) 225. 
[68] S.M. Bilenky and B. Pontecorvo, Lett. Nuovo Cim. 17 (1976) 569.

[69] K.S. Hirata et al., Phys. Rev. Lett. 63 (1989) 16.

[70] K. Hirata et al., Phys. Rev. Lett. 58 (1987) 1490.

[71] P. Minkowski, Phys. Lett. B 67 (1977) 421; M. Gell-Mann, P. Ramond and R. Slansky, in Supergravity, p. 315, edited by F. van Nieuwenhuizen and D. Freedman, North Holland, Amsterdam, (1979); T. Yanagida, Proc. of the Workshop on Unified Theory and the Baryon Number of the Universe, KEK, Japan, (1979); S.L. Glashow, NATO Adv.Study Inst. Ser. B Phys. 59 (1979) 687; R.N. Mohapatra and G. Senjanović, Phys. Rev. D23 (1981) 165.

[72] P. Anselmann et al. (GALLEX Collaboration), Phys. Lett. B327 (1994) 377.

[73] J.N. Abdurashitov et al. (SAGE Collaboration), Phys. Lett. B328 (1994) 234.

[74] L. Wolfenstein, Phys. Rev. D17 (1978) 2369; S. P. Mikheev and A. Yu. Smirnov, Nuovo Cim. C9 (1986) 17.

[75] Y. Fukuda et al. (Super-Kamiokande Collaboration), Phys. Rev. Lett. 81 (1998) 1562. R. Wendell et al. (Super-Kamiokande Collaboration), Phys.Rev. D81 (2010) 092004.

[76] Q.R. Ahmad et al. (SNO Collaboration), Phys. Rev. Lett. 89 (2002) 011301: B. Aharmim et al (SNO Collaboration) arXiv:1109.0763.

[77] K. Eguchi et al. (KamLAND Collaboration), Phys. Rev. Lett. 90 (2003) 021802;T. Araki et al. (KamLAND Collaboration), Phys.Rev.Lett.94 (2005) 081801. Abe, S. et al. (KamLAND Collaboration), Phys. Rev. Lett. 100 (2008) 221803.

[78] M.H. Ahn et al. (K2K Collaboration), Phys. Rev. Lett. 90 (2003) 041801.

[79] D.G. Michael et al. (MINOS Collaboration), Phys. Rev. Lett. 97 (2006) 191801. P. Adamson et al. (MINOS Collaboration), Phys. Rev. Lett. 106 (2011) 181801. 
[80] M. Apollonio et al. (CHOOZ Collaboration) Eur. Phys. J. C27 (2003) 331.

[81] K.Abe et al. (T2K Collaboration), Phys. Rev. Lett. 107 (2011) 041801.

[82] M. Gunther et al. (Heidelberg - Moscow Collaboration), Phys. Rev.D55 (1997) 54.

[83] A. Aguilar et al. (LSND Collaboration), Phys. Rev. D64 (2001) 112007.

[84] Y. Abe et al. (Double Chooz Collaboration), arXiv:1112.6353v3.

[85] F. P. An et al. (Daya Bay Collaboration), Phys. Rev. Lett. 108 (2012) 171803; arXiv:1203.1669v1.

[86] J. Ahn et al. (Reno Collaboration), Phys. Rev. Lett. 108 (2012) 191802.

[87] S. Davidson, E. Nardi, Y. Nir, Phys. Rept. 466, 105 (2008).

[88] G. Senjanovic, arXiv:1012.4104. 